\title{
A staggered high-dimensional Proper Generalised Decomposition for coupled magneto-mechanical problems with application to MRI scanners
}

\author{
G. Barroso ${ }^{a}$, M. Seoane ${ }^{b, a}$, A. J. Gil ${ }^{a}$, P. D. Ledger ${ }^{a}$, M. Mallett $^{b}$, A. Huerta $^{c}$ \\ (a) Zienkiewicz Centre for Computational Engineering, College of Engineering \\ Swansea University, Bay Campus, SA1 8EN, United Kingdom \\ \{878809,922435,a.j.gil, p.d.ledger\}@swansea.ac.uk \\ (b) Siemens Healthineers, MR Magnet Technology, \\ Wharf Road, Eynsham, Witney, Oxon OX29 4BP, United Kingdom \\ $\{$ marcos.seoane_choucino, michael.mallett\}@siemens-healthineers.com \\ (c) Laboratori de Càlcul Numèric (LaCàN), Universitat Politècnica de Catalunya \\ c/ Jordi Girona 1-3 Edifici C2, 08034, Barcelona, Spain \\ antonio.huerta@upc.edu
}

\begin{abstract}
Manufacturing new Magnetic Resonance Imaging (MRI) scanners represents a computational challenge to industry, due to the large variability in material parameters and geometrical configurations that need to be tested during the early design phase. This process can be highly optimised through the employment of user-friendly computational metamodels constructed on the basis of Reduced Order Modelling (ROM) techniques, where high-dimensional parametric offline solutions are obtained, stored and assimilated in order to be efficiently queried in real time. This paper presents a novel Proper Generalised Decomposition (PGD) based metamodel for the analysis of electro-magneto-mechanical interactions in the context of MRI scanner design, with three distinct novelties. First, the paper derives, from scratch, a five-dimensional parametrised offline solution process, expressed in terms of (axisymmetric) cylindrical coordinates, external excitation frequency, electrical conductivity of the embedded shields and strength of the static magnetic field. Second, by exploiting the staggered nature of the coupled problem at hand, an efficient sequential PGD algorithm is derived and compared against a previously published monolithic PGD algorithm. As a third novelty, the paper draws some interesting comparisons against an alternative tailor-made ROM technique, where the electromagnetic equations are solved using a Proper Orthogonal Decomposition model. A series of numerical examples are presented in order to illustrate, motivate and demonstrate the validity and potential of the considered approach, especially in terms of cost reduction.
\end{abstract}

Keywords: Coupled magneto-mechanical problems, MRI scanners, Design optimisation, Reduced Order Modelling, Proper Generalised Decomposition, Real time simulation, Multiple-query evaluation

\section{Introduction}

The use of Magnetic Resonance Imaging (MRI) [1] as part of the decision process within a medical and/or clinical environment has become standard practice, due to scanners' high inbuilt resolution when imaging fractures [2], joints [3] and soft tissues, such as damaged cartilage [2] or tumours [4]. An additional advantage of MRI is its non-intrusive nature [5], meaning that no harmful ionising radiation is used, minimising the possibility of any secondary radiation effects on the patient. As such, MRI is particularly recommended to patients requiring multiple imaging examinations. 
A prototypical MRI scanner, see Figure 1, consists of a cryostat enclosing three fundamental components: main superconducting Direct Current (DC) coils, gradient Alternating Current (AC) coils and radiation shields. The main DC coils [6] are immersed in a supercooled vessel of liquid helium and their purpose is the generation of a strong background stationary magnetic field. Gradient AC coils emit dynamic electromagnetic pulses that interact with the protons of the patient's body. An image can be generated by post-processing this interaction [5]. Radiation shields typically consist of three conducting components, the Outer Vacuum Chamber (OVC) shield, the $77 \mathrm{~K}$ radiation shield $(77 \mathrm{~K})$ and the $4 \mathrm{~K}$ helium vessel shield $(4 \mathrm{~K})$, which are used to prevent radiation from escaping through.

Two main physics are considered in order to simulate the complex physical interactions and coupling within an MRI scanner: electromagnetics and mechanics. The electromagnetic problem is often represented by the eddy current approximation [7-10] of the general Maxwell's equations when dealing with relatively low frequencies with respect to the high electrical conductivities of the conducting components. The mechanical problem is modelled using the well-known linear elasticity theory [11], assuming small displacements but not necessarily small velocities or accelerations [12]. The reader is referred to [13-16] for a thorough explanation of the different modelisation assumptions.
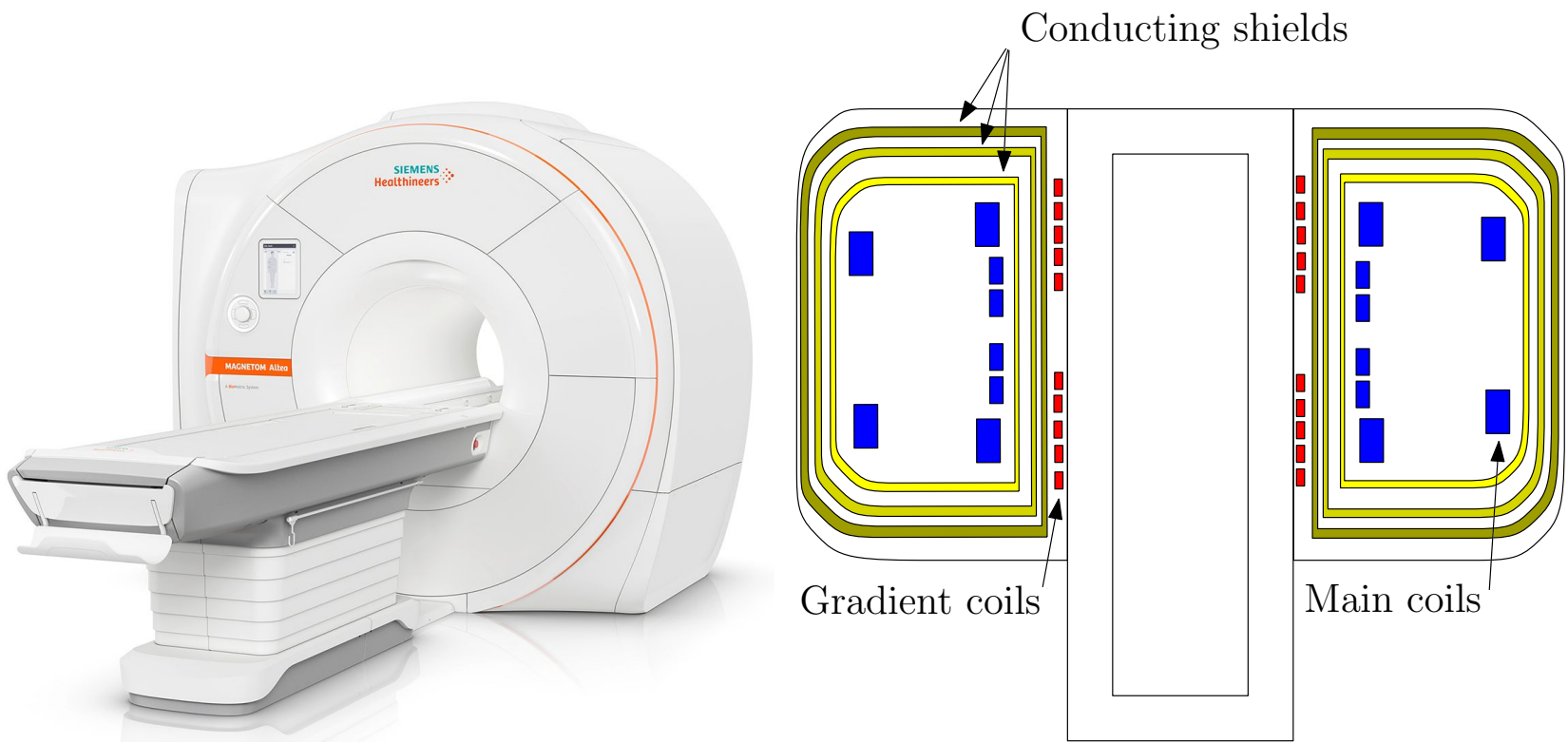

Figure 1: MRI scanner description; new MRI device model MAGNETOM Altea 1.5T Open Bore system, courtesy of Siemens Healthineers.

According to Siemens Healthineers, one of the bottlenecks when manufacturing a new MRI scanner, such as the recent ultra high-field 7 Tesla (7T) magnet, is the computational effort required during the design phase [17]. A crucial part of this involves the simulation of a large number of electro-magneto-mechanical problems with slight variations in the geometry and/or material parameters. The design stage can thus become very time consuming, which eventually is translated into an overall manufacturing cost increase. Reduced Order Modelling (ROM) techniques have recently gained momentum to help speed up the design optimisation process. ROM can be used in order to construct computational metamodels capable of interacting with the user in real time via multi-parametric approximations of the so-called full order solution [18]. Crucially, these multi-parametric approximations must be carefully obtained in order to avoid the well-known curse of dimensionality [19], present when attempting to solve higherdimensional (i.e. 4D, 5D) problems through standard discretisation techniques (i.e. Finite Element Method (FEM)). An additional benefit of ROM is the speed of interaction with the user during the online stage, facilitating real time multiple-query optimisation. In this case, 
the computational effort lies on the marginal cost of another input-output evaluation and an increased precomputation cost is acceptable [20].

Within the generic name of ROM, there is a wealth of modelling strategies. Among them, $a$ posteriori ROM techniques rely on the use of reduced approximation bases (modes), carefully extracted after (typically) carrying out a Truncated Singular Value Decomposition (TSVD) on a set of representative snapshots, as in the Proper Orthogonal Decomposition (POD) method $[21,22]$. The most appealing property of POD is its orthogonality. Indeed when seeking online solutions dependent upon a maximum of two parameters, the singular value decomposition needed in order to generate the POD modes is known to be optimal [23], with no other ROM method capable of producing a better approximation with a smaller number of modes. Unfortunately, this is not necessarily the case when incorporating further parameters into the online solution. In addition, the correct choice of relevant snapshots for the construction of the TSVD is by no means a trivial task and, moreover, POD still requires the assembly and solution of a reduced system of equations in the online stage.

Alternatively, a priori ROM methods, such as the Proper Generalised Decomposition (PGD) method [19], do not require any previously stored information of the response of the system (i.e. snapshots). They build a higher-dimensional parametric offline solution and, subsequently, during the online stage, a simple interpolation of this parametric solution is performed. The intrusivity of the offline stage [24] is counterbalanced by the speed of the online stage, allowing for user queries to be responded in real time. Moreover, the PGD technique allows to compute sensitivity maps because an explicit generalised solution is available. This PGD methodology has been successfully implemented in numerous applications, such as Helmholtz based problems [25, 26], solid mechanics [27], power distribution systems [28], flow problems [24, 29, 30], thermal problems [31, 32], degenerated 3D domains such as plates and shells [33] and also with geometrical parametrisations for heat problems [34].

The first main contribution of this paper is the extension of the frequency-based PGD methodology presented in [35] to a higher-dimensional parametric space, including also the strength of the background static magnetic field and the electrical conductivity of the shielding components as extra parameters of interest. As a second contribution, and also departing from our previous work in [35], this paper exploits the staggered nature of the coupled electromagneto-mechanical equations by designing a sequential PGD algorithm where the electromagnetic and mechanical equations are solved in a staggered fashion. Numerical results will illustrate that this new approach is capable of greatly increasing the robustness of the overall algorithm. Finally, the a priori PGD method will be compared to the a posteriori POD technique applied to the electromagnetic problem, following the methodology recently introduced in [36] and adapted in this paper for axi-symmetric scenarios. This comparison will be used in order to draw some interesting conclusions from the computational design (especially in terms of cost) standpoint.

The paper is structured as follows. Section 2 briefly presents the two physics governing the behaviour of the problem, the appropriate set of transmission, boundary and initial conditions, and the computational treatment of the problem, resulting into the so-called full order model. Section 3 provides a detailed derivation of the new staggered high-dimensional PGD method and its application to electromagnetics and mechanics, the two physics of the problem. A brief description of the combined POD-full order technique is presented in Section 4 . Section 5 provides a comprehensive set of numerical results for two different geometries, first a simplified (test) magnet followed by a more realistic MRI scanner configuration. In this section, the true power of the PGD method is demonstrated by depicting field quantities of interest (i.e. magnetic potential, eddy current distribution) across the entire computational domain queried in real time. The conclusions of the paper are found in Section 6, followed by two appendices including important implementation details, added for completeness and to facilitate reproducibility of 
results.

\section{Full order model description}

In order to computationally simulate the electromagnetic interactions taking place in MRI scanners, the underlying non-linear coupled magneto-mechanical problem, see Figure 2, is formulated in a Lagrangian setting [36], with the magnetic vector potential $\boldsymbol{A}$ and the mechanical displacements $\boldsymbol{u}$ as solution (unknown) fields.

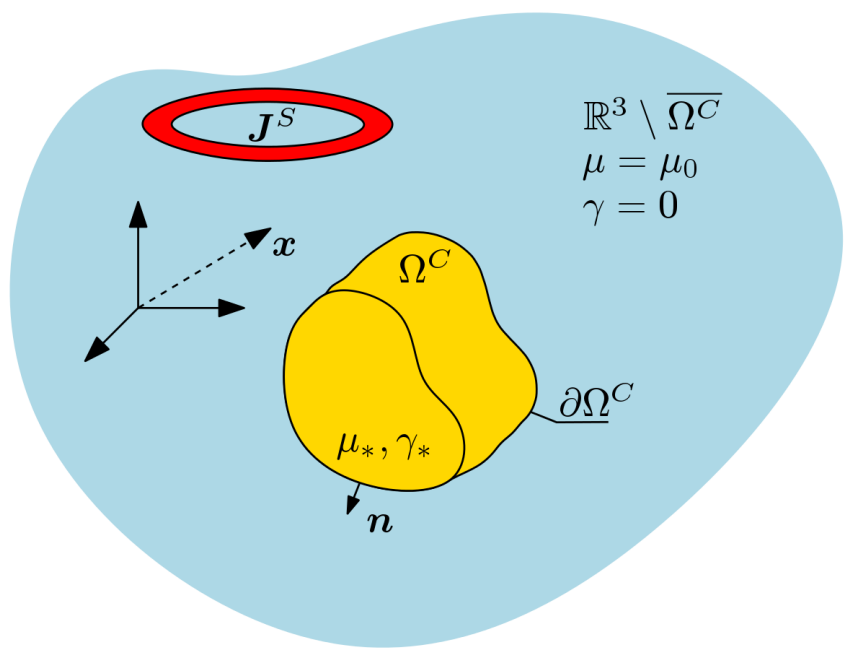

Figure 2: Description of a general magneto-mechanic problem; conducting component $\Omega^{C}$ (with magnetic permeability $\mu=\mu_{*}$ and electrical conductivity $\gamma=\gamma_{*}$ ) in a non-conducting three dimensional space $\mathbb{R}^{3} \backslash \overline{\Omega^{C}}$ (with $\mu=\mu_{0}$ and $\gamma=0$ ). Problem excited by a current source $\boldsymbol{J}^{S}(t)=\boldsymbol{J}^{D C}+\operatorname{Re}\left(\boldsymbol{g}^{A C} e^{\mathrm{i} \omega t}\right)$ prescribed in a series of coils.

By linearising about the static (DC) solution, the transient (AC) problem becomes linear in time and it can thus be solved in time-harmonic variables as

$$
\begin{aligned}
\boldsymbol{A}(t) & =\boldsymbol{A}^{D C}+\operatorname{Re}\left(\boldsymbol{\mathcal { A }}^{A C} e^{\mathrm{i} \omega t}\right), \\
\boldsymbol{u}(t) & =\boldsymbol{u}^{D C}+\operatorname{Re}\left(\boldsymbol{u}^{A C} e^{\mathrm{i} \omega t}\right),
\end{aligned}
$$

where $\mathrm{i}:=\sqrt{-1}, \omega=2 \pi f$ represents the angular frequency of the harmonic excitation and $f$ is the frequency in Hertz. The governing equations for the linearised transient problem are thoroughly derived in [37] and the resulting strong form is defined as: Find $\left(\boldsymbol{A}^{A C}, \boldsymbol{u}^{A C}\right) \in$ $\left(\mathbb{C}^{3} \times \mathbb{C}^{3}\right)$ such that

$$
\begin{aligned}
& \operatorname{curl}\left(\mu^{-1} \operatorname{curl} \mathcal{A}^{A C}\right)+\mathrm{i} \omega \gamma \boldsymbol{A}^{A C}=\boldsymbol{g}^{A C} \\
& \operatorname{div} \mathcal{A}^{A C}=0 \\
& \operatorname{div}\left(\boldsymbol{\sigma}^{m}\left(\boldsymbol{u}^{A C}\right)+\mu^{-1} \boldsymbol{T}\left(\boldsymbol{A}^{D C}, \boldsymbol{A}^{A C}\right)\right)=-\rho \omega^{2} \boldsymbol{u}^{A C} \\
& \boldsymbol{A}^{A C}=O\left(|\boldsymbol{x}|^{-1}\right) \\
& \boldsymbol{u}^{A C}=\mathfrak{u}_{D}^{A C} \\
& \boldsymbol{n} \times\left[\mathcal{A}^{A C}\right]_{\partial \Omega^{C}}=\mathbf{0} \\
& \boldsymbol{n} \times\left[\mu^{-1} \operatorname{curl} \boldsymbol{A}^{A C}\right]_{\partial \Omega^{C}}=\mathbf{0} \\
& \left.\left.\left(\boldsymbol{\sigma}^{m}\left(\boldsymbol{u}^{A C}\right)+\mu^{-1} \boldsymbol{T}\left(\boldsymbol{A}^{D C}, \boldsymbol{A}^{A C}\right)\right)\right|_{\partial \Omega^{C}} ^{-} \boldsymbol{n}=\mu^{-1} \boldsymbol{T}\left(\boldsymbol{A}^{D C}, \boldsymbol{A}^{A C}\right)\right)\left.\right|_{\partial \Omega^{C}} ^{+} \boldsymbol{n} \quad \text { on } \partial \Omega^{C},
\end{aligned}
$$

where $\mu, \gamma$ and $\rho$ are the magnetic permeability, the electrical conductivity and the material density, respectively; $\boldsymbol{n}$ is the outward normal vector (pointing from the conducting to the 
non-conducting side); $\boldsymbol{\sigma}^{m}\left(\boldsymbol{u}^{A C}\right)$ in (2c) represents the mechanical contribution to the Cauchy stress tensor and $\left.\mu^{-1} \boldsymbol{T}\left(\boldsymbol{A}^{D C}, \boldsymbol{A}^{A C}\right)\right)$ in (2c) and (2h) represents the linearised Maxwell stress tensor. Once the linearised transient problem (2) is solved, the Eulerian complex amplitudes of the electric and magnetic AC fields can be computed as

$$
\begin{aligned}
\boldsymbol{E}^{A C} & =-\mathrm{i} \omega \boldsymbol{\mathcal { A }}^{A C}+\mathrm{i} \omega \boldsymbol{B}_{0}^{D C} \times \boldsymbol{U}^{A C} & & \text { in } \Omega^{C} \\
\boldsymbol{H}^{A C} & =\mu^{-1} \boldsymbol{B}_{0}^{A C}=\mu^{-1} \operatorname{curl} \boldsymbol{A}^{A C} & & \text { in } \mathbb{R}^{3}
\end{aligned}
$$

and the electric and magnetic time varying quantities are recovered as

$$
\begin{array}{ll}
\boldsymbol{E}=\operatorname{Re}\left(\boldsymbol{E}^{A C} e^{\mathrm{i} \omega t}\right)=\operatorname{Re}\left(\left(-\mathrm{i} \omega \mathcal{A}^{A C}+\mathrm{i} \omega \boldsymbol{B}_{0}^{D C} \times \boldsymbol{u}^{A C}\right) e^{\mathrm{i} \omega t}\right) & \text { in } \Omega^{C} \\
\boldsymbol{H}=\boldsymbol{H}^{D C}+\operatorname{Re}\left(\mathcal{H}^{A C} e^{\mathrm{i} \omega t}\right)=\mu^{-1}\left(\operatorname{curl} \boldsymbol{A}^{D C}+\operatorname{Re}\left(\left(\operatorname{curl} \mathcal{A}^{A C}\right) e^{\mathrm{i} \omega t}\right)\right) & \text { in } \mathbb{R}^{3}
\end{array}
$$

For the purpose of this paper, we assume that the fields $\boldsymbol{A}^{D C}, \boldsymbol{B}_{0}^{D C}=\operatorname{curl} \boldsymbol{A}^{D C}$ and $\boldsymbol{u}^{D C}$ are assumed to be known and independent of the model parameters that we wish to vary. These fields can be computed by following the procedure in $[14,15,37]$. The focus of this paper is restricted to axisymmetric configurations and thus the problem is formulated in cylindrical $(r, \phi, z)$ coordinates across the meridian two-dimensional $(r, z)$ plane $\Omega_{p}$ (refer to [35] for a diagrammatic representation). The unbounded domain is truncated at a finite distance away from the conducting embedded domain $\Omega_{p}^{C}$ and as an approximation to the decay condition we set $\mathcal{A}_{\phi}=0$ on $\partial \Omega_{p}$. The transient current source is assumed to have only an angular component $\boldsymbol{g}^{A C}(r, \phi, z)=g_{\phi}^{S}(r, z) \boldsymbol{e}_{\phi}$ and, therefore, the solution fields become ${ }^{1}$

$$
\begin{aligned}
\boldsymbol{A}^{A C} & =r \mathcal{A}_{\phi}(r, z) \boldsymbol{e}_{\phi}, \\
\boldsymbol{u}^{A C} & =\boldsymbol{u}=r \mathcal{U}_{r}(r, z) \boldsymbol{e}_{r}+\mathcal{U}_{z}(r, z) \boldsymbol{e}_{z}
\end{aligned}
$$

where $\boldsymbol{e}_{r}, \boldsymbol{e}_{\phi}$ and $\boldsymbol{e}_{z}$ represent the unit basis vectors of the cylindrical system. Note that the fields $\mathcal{A}_{\phi}$ and $\mathcal{U}_{r}$ have been scaled in order to avoid singularities along the $r=0$ axis. The reader is referred to $[13,37]$ for further details on the derivation of the axisymmetric formulation.

\subsection{Full order weak formulation}

The so-called full order electromagnetic AC problem is obtained as the standard weak form of the strong form (2) set in an axisymmetric configuration $[13,37]$ as: Find $\mathcal{A}_{\phi}(r, z) \in X(0)$ such that

$$
W^{A}\left(\mathcal{A}_{\phi}, \delta \mathcal{A}_{\phi}\right)=S^{A}\left(\delta \mathcal{A}_{\phi}\right) \quad \forall \delta \mathcal{A}_{\phi} \in X(0)
$$

where

$$
W^{A}\left(\mathcal{A}_{\phi}, \delta \mathcal{A}_{\phi}\right)=W_{K}^{A}\left(\mathcal{A}_{\phi}, \delta \mathcal{A}_{\phi}\right)+\mathrm{i} \omega W_{C}^{A}\left(\mathcal{A}_{\phi}, \delta \mathcal{A}_{\phi}\right)
$$

with

$$
\begin{aligned}
W_{K}^{A}(a, b) & :=\int_{\Omega_{p}} \frac{\mu^{-1}}{r} \nabla_{p}\left(r^{2} a\right) \cdot \nabla_{p}\left(r^{2} \bar{b}\right) \mathrm{d} \Omega \\
W_{C}^{A}(a, b) & :=\int_{\Omega_{p}^{C}} \gamma a \bar{b} r^{3} \mathrm{~d} \Omega \\
S^{A}(b) & :=\int_{\Omega_{p}} g_{\phi}^{S} \bar{b} r^{2} \mathrm{~d} \Omega .
\end{aligned}
$$

\footnotetext{
${ }^{1}$ Note that the upper index $A C$ for both fields $\mathcal{A}_{\phi}$ and $\boldsymbol{u}$ is dropped hereafter for simplicity.
} 
Note that the space $X(0)$ used in (6) is defined as

$$
X(0):=\left\{\mathcal{A}_{\phi}: \mathcal{A}_{\phi} \in H^{1}\left(\Omega_{p}\right), \mathcal{A}_{\phi}=0 \text { on } \partial \Omega_{p}\right\}
$$

and the gradient of the scalar potential in the meridian plane is defined as $\nabla_{p} \mathcal{A}_{\phi}:=\frac{\partial \mathcal{A}_{\phi}}{\partial r} \boldsymbol{e}_{r}+$ $\frac{\partial \mathcal{A}_{\phi}}{\partial z} \boldsymbol{e}_{z}$. Similarly, the full order axisymmetric mechanical AC problem [13, 37] is also obtained from (2) as: Find $\boldsymbol{u}(r, z) \in Y\left(\boldsymbol{u}_{D}\right)$ such that

$$
W^{u}(\boldsymbol{u}, \delta \boldsymbol{u})=-S^{u}\left(\mathcal{A}_{\phi}, \delta \boldsymbol{u}\right) \quad \forall \delta \boldsymbol{u} \in Y(\mathbf{0})
$$

where

$$
W^{u}(\boldsymbol{u}, \delta \boldsymbol{u})=W_{K}^{u}(\boldsymbol{u}, \delta \boldsymbol{u})+\mathrm{i} \omega^{2} W_{C}^{u}(\boldsymbol{u}, \delta \boldsymbol{u})-\omega^{2} W_{M}^{u}(\boldsymbol{u}, \delta \boldsymbol{u})
$$

with

$$
\begin{aligned}
W_{K}^{u}(\boldsymbol{a}, \boldsymbol{b}) & :=\int_{\Omega_{p}^{C}} \boldsymbol{\sigma}^{m}(\boldsymbol{a}): \nabla \overline{\boldsymbol{b}} r \mathrm{~d} \Omega, \\
W_{C}^{u}(\boldsymbol{a}, \boldsymbol{b}) & :=2 \xi \int_{\Omega_{p}^{C}} \rho \boldsymbol{a} \cdot \overline{\boldsymbol{b}} r \mathrm{~d} \Omega, \\
W_{M}^{u}(\boldsymbol{a}, \boldsymbol{b}) & :=\int_{\Omega_{p}^{C}} \rho \boldsymbol{a} \cdot \overline{\boldsymbol{b}} r \mathrm{~d} \Omega, \\
S^{u}(a, \boldsymbol{b}) & :=\int_{\Omega_{p}^{C}} \mu^{-1} \boldsymbol{T}\left(\mathcal{A}_{\phi}^{D C}, a\right): \nabla \overline{\boldsymbol{b}} r \mathrm{~d} \Omega-\left.\int_{\partial \Omega_{p}^{C, N}} \mu_{0}^{-1} \boldsymbol{T}\left(\mathcal{A}_{\phi}^{D C}, a\right)\right|^{+} \boldsymbol{n} \cdot \overline{\boldsymbol{b}} r \mathrm{~d} S,
\end{aligned}
$$

where the mass proportional Rayleigh damping coefficient [11] defined as $\alpha_{M}:=2 \omega \xi$ has been used in (12), being $\xi$ a dimensionless ratio that allows to control the amount of damping added to the system and

$$
Y\left(\boldsymbol{u}_{D}\right):=\left\{\boldsymbol{u}: \boldsymbol{u} \in\left(H^{1}\left(\Omega_{p}^{C}\right)\right)^{2}, \boldsymbol{u}=\boldsymbol{u}_{D} \text { on } \partial \Omega_{p, D}^{C}\right\}
$$

The coupled magneto-mechanical problem (6)-(10) is then discretised in space and solved using a high order FEM Galerkin approximation [11] to obtain discrete values of the solution fields. For further details, the reader is referred to $[14,15]$.

\section{Staggered high-dimensional Proper Generalised Decomposition (PGD) formu- lation}

As mentioned in Section 1, the design process of MRI scanners can be highly optimised by incorporating material and geometrical parameters as part of a high-dimensional parametric solution and, thus, the general PGD methodology is first introduced in this section with this aim. Subsequently, the chosen design (optimisation) parameters are motivated and discussed in terms of their computational implementation. Finally, a novel staggered PGD solver is described and the PGD formulation is particularised for the two physics of interest, namely electromagnetics and mechanics.

\subsection{High-dimensional parametric PGD formulation}

The PGD method is presented for the general case of a high-dimensional parametric problem that considers the spatial two-dimensional domain $\Omega_{p}$ and a $d$-dimensional (tensorial) parametric space $\Omega_{q}=\Omega_{w_{1}} \times \cdots \times \Omega_{w_{d}}$. If the solution vector field of this problem is formulated as $\boldsymbol{q}=\boldsymbol{q}\left(r, z, w_{1}, \ldots, w_{d}\right)$, the core idea of the PGD methodology [19] is the assumption that the 
vector field $\boldsymbol{q}$ can be approximated using a separable representation $\boldsymbol{q}^{N}=\boldsymbol{q}^{N}\left(r, z, w_{1}, \ldots, w_{d}\right)$ as

$$
\boldsymbol{q}\left(r, z, w_{1}, \ldots, w_{d}\right) \approx \boldsymbol{q}^{N}\left(r, z, w_{1}, \ldots, w_{d}\right):=\sum_{n=1}^{N} \beta^{n} \mathcal{F}^{n}(r, z) \prod_{i=1}^{d} \mathcal{G}_{i}^{n}\left(w_{i}\right),
$$

where $N$ denotes the total number of so-called modes, multiplicatively decomposed into spatial and parametric modes. The spatial modes $\mathcal{F}^{n}(r, z)$ are represented by normalised vectorial functions whilst the parametric modes $\mathcal{G}_{i}^{n}\left(w_{i}\right)$ are denoted by normalised scalar functions and the mode weights $\beta^{n}$ are scalar coefficients computed via an $L^{2}$ projection once all spatial and parametric modes are known [25]. Note that the choice made in this paper is to represent the spatial $\Omega_{p}$ related modes with a single function $\mathcal{F}^{n}(r, z)$ while the parametric domain $\Omega_{q}$ related modes are represented by the product of a set of separated scalar functions $\prod_{i=1}^{d} \mathcal{G}_{i}^{n}\left(w_{i}\right)$. Although it is also possible to multiplicatively decompose the spatial domain related modes into two different functions for $r$ and $z$ [38], the choice adopted in this paper is to use a single function so that more complex geometries can be handled.

In (14), each mode $n$ is sequentially computed using a Greedy algorithm [39]. For a given $n^{\text {th }}$ mode, the accumulated solution $\boldsymbol{q}^{n}=\boldsymbol{q}^{n}\left(r, z, w_{1}, \ldots, w_{d}\right)$ may be expressed as

$$
\begin{aligned}
\boldsymbol{q}^{n}\left(r, z, w_{1}, \ldots, w_{d}\right) & :=\sum_{m=1}^{n-1} \beta^{m} \mathcal{F}^{m}(r, z) \prod_{i=1}^{d} \mathcal{G}_{i}^{m}\left(w_{i}\right)+\boldsymbol{f}(r, z) \prod_{i=1}^{d} g_{i}\left(w_{i}\right) \\
& =\boldsymbol{q}^{n-1}\left(r, z, w_{1}, \ldots, w_{d}\right)+\boldsymbol{f}(r, z) \prod_{i=1}^{d} g_{i}\left(w_{i}\right),
\end{aligned}
$$

and, by using a fixed-point Alternating Direction Scheme (ADS) algorithm [19], the approximation term $\boldsymbol{f}(r, z) \prod_{i=1}^{d} g_{i}\left(w_{i}\right)$ (lowercase) is assumed to converge to $\beta^{n} \mathcal{F}^{n}(r, z) \prod_{i=1}^{d} \mathcal{G}_{i}^{n}\left(w_{i}\right)$ (uppercase) by sequentially updating each individual component function (i.e. $\left\{\boldsymbol{f}(r, z), g_{1}\left(w_{1}\right) \ldots\right.$ $\left.g_{d}\left(w_{d}\right)\right\}$ ) while assuming the others known. A graphical representation of this generic highdimensional PGD algorithm ca ne found in [35] (for specific details regarding the FEM discretisation and thus the nomenclature depicted in the algorithm, the reader is referred to Appendix $\mathrm{A}$ and $[35])$.

\subsection{Parameters of interest: the high-dimensional parametric space}

A novel frequency-based PGD technique was recently developed in [35] in order to conduct real time evaluations of the performance of an MRI scanner when subjected to frequency dependent magnetic excitations. In this paper, we aim to greatly enhance the flexibility of this PGD metamodel, by incorporating further parameters of interest into the query process, namely, the angular frequency $\omega$, the electrical conductivity $\gamma$ and the strength of the static magnetic field $B_{0}$, computed as $B_{0}=\max \left(\left|\boldsymbol{B}_{0}^{D C}\right|_{r=0} \mid\right)$. With this in mind, the solution domain $\Omega$ is expressed as the tensorial product of the spatial meridian $(r, z)$ space $\Omega_{p}$ times the parametric domain $\Omega_{q}$ as $\Omega=\Omega_{p} \times \Omega_{q}$. Whilst in [35] the parametric domain exclusively contained the one-dimensional frequency domain $\Omega_{q}=\Omega_{\omega}$, in this paper the parameteric domain is extended to further incorporate the electrical conductivity and the strength of the magnetic field as $\Omega_{q}=\Omega_{\omega} \times \Omega_{\gamma} \times \Omega_{B_{0}}$.

The conductivity of the embedded shields is a key parameter that is typically modified (in a continuous fashion) during the design stage of an MRI scanner. The approach followed in this paper consists of introducing a scalar coefficient $\alpha_{\gamma} \in[0.5,2]$ that scales the reference conductivities $\gamma$ of all shields. In addition, the strength of the static magnetic field $B_{0}$, measured in Teslas [T], refers to the maximum value of the magnitude of the background static magnetic field $\boldsymbol{B}_{0}^{D C}$ along the $r=0$ axis. This parameter is used to classify MRI scanners and indicate the overall strength of the equipment, with typical commercial values for $B_{0}$ currently at $1.5 \mathrm{~T}$, 
$3 \mathrm{~T}$ and 7T. Therefore, a further scalar coefficient $\alpha_{B_{0}} \in[1,7]$ is introduced in order to scale the strength of the static field $\boldsymbol{B}_{0}^{D C}$. Finally, the dependency of the solution field $\boldsymbol{q}$ upon the high-dimensional parametric space will be denoted as $\boldsymbol{q}=\boldsymbol{q}\left(r, z, \omega, \gamma, B_{0}\right)$.

Due to the staggered nature of the coupled weak forms (6) and (10), we advocate in this paper for a sequential solution procedure where the electromagnetic governing equations are solved first (in terms of the magnetic vector potential) followed by the solution of the mechanical governing equations (in terms of the displacement field). This staggered solution procedure will be shown to greatly enhance the computational convergence (i.e. robustness and speed) of the PGD algorithm, especially when considering more than a single parameter of interest (as it was the case in [35]).

As it was already shown in our previous work [14-16, 35], when the model is excited at frequencies in the neighbourhood of the resonant modes, singularities can arise in the solution fields and outputs of interest. Due to the existence of these resonant phenomena, Reference [35] introduced an automatic regularised-adaptive frequency PGD splitting technique in order to accurately capture the location of the resonant modes. In [35], this splitting procedure was shown to be fundamental ${ }^{2}$ in order to reconstruct, in real time, frequency response spectra for a series of (integrated) magnitudes of industrial interest (i.e. kinetic energy and dissipated power). Naturally, when employing a sequential PGD solution strategy, as will be the case in this work, different frequency (splitting) intervals can be considered when solving the electromagnetic (domain $\Omega_{\omega}^{A}$ ) and mechanical (domain $\Omega_{\omega}^{u}$ ) physics, separately. This is diagrammatically depicted in Figure 3 where, as an example, for a single frequency domain of interest in the electromagnetics problem, namely $\Omega_{\omega}^{A}$, three frequency intervals $\Omega_{\omega}^{u, 1}, \Omega_{\omega}^{u, 2}$ and $\Omega_{\omega}^{u, 3}$ are used for the mechanical problem. This will always be the case, namely, the frequency range requires a higher level of discretisation (and possibly splitting) for the solution of the mechanical equations than for the solution of the electromagnetic equations (due to the presence of singularities in the former equations). This important consideration implies that non-matching parametric (frequency) meshes might be necessary when using the PGD algorithm in both physics separately. This will require the use of one-dimensional mortar integrals, see Section 3.5 and Appendix B for further details.

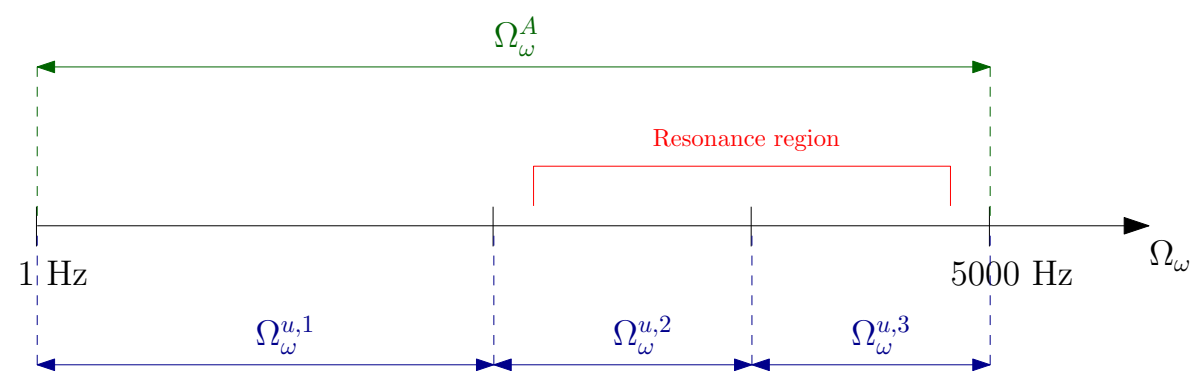

Figure 3: Different parametric domains for electromagnetics and mechanics due to adaptive splitting PGD technique.

\subsection{Augmented full order model}

Prior to the formulation of the PGD methodology, the full order model (see Section 2) must be reformulated in the augmented higher-dimensional space $\Omega_{p} \times \Omega_{q}$. Electromagnetics and mechanics augmented weak form problems are presented in what follows.

\footnotetext{
${ }^{2} \mathrm{As}$ it is expected, these sharp changes in the kinetic energy and dissipated power can make extremely challenging the convergence of the PGD algorithm due to the ill-conditioning of the resulting PGD system in the proximity of these resonant modes.
} 


\subsubsection{Electromagnetics}

The axisymmetric weak form of the electromagnetic problem is formulated in the highdimensional space $\Omega_{p} \times \Omega_{q}^{A}$ (the upper index $(\cdot)^{A}$ is used to emphasise the electromagnetics parametric domain). Note that there is no dependency on $B_{0}$ in the electromagnetic weak formulation (6) and thus the electromagnetic parametric domain can be denoted as $\Omega_{q}^{A}=$ $\Omega_{\omega}^{A} \times \Omega_{\gamma}^{A}$. The high-dimensional version of the full order weak form (6) can be written as: Find $\mathcal{A}_{\phi}(r, z, \omega, \gamma) \in \mathcal{X}(0)$ such that ${ }^{3}$

$$
\mathcal{W}_{K}^{A}\left(\mathcal{A}_{\phi}, \delta \mathcal{A}_{\phi}\right)+\mathrm{i} \mathcal{W}_{C}^{A}\left(\mathcal{A}_{\phi}, \delta \mathcal{A}_{\phi}\right)=\mathcal{S}^{A}\left(\delta \mathcal{A}_{\phi}\right) \quad \forall \delta \mathcal{A}_{\phi} \in \mathcal{X}(0)
$$

with

$$
\begin{aligned}
\mathcal{W}_{K}^{A}(a, b) & :=\int_{\Omega_{\gamma}^{A}} \int_{\Omega_{\omega}^{A}} W_{K}^{A}(a, b) \mathrm{d} \omega \mathrm{d} \gamma \\
\mathcal{W}_{C}^{A}(a, b) & :=\int_{\Omega_{\gamma}^{A}} \alpha_{\gamma} \int_{\Omega_{\omega}^{A}} \omega W_{C}^{A}(a, b) \mathrm{d} \omega \mathrm{d} \gamma, \\
\mathcal{S}^{A}(b) & :=\int_{\Omega_{\gamma}^{A}} \int_{\Omega_{\omega}^{A}} S^{A}(b) \mathrm{d} \omega \mathrm{d} \gamma,
\end{aligned}
$$

and

$$
\mathcal{X}(0):=\left\{\mathcal{A}_{\phi}: \mathcal{A}_{\phi} \in H^{1}\left(\Omega_{p} \times \Omega_{\omega}^{A} \times \Omega_{\gamma}^{A}\right), \mathcal{A}_{\phi}=0 \text { on } \partial \Omega_{p} \times \Omega_{\omega}^{A} \times \Omega_{\gamma}^{A}\right\} .
$$

\subsubsection{Mechanics}

In the context of a typical MRI scanner configuration, it is often considered that the conducting shields are isolated from each other, see Figure 4. Therefore, when solving the mechanical governing equations, the shields can be solved individually (in parallel) and then proceed to assemble the mechanical response of the MRI system all together if needed. With this in mind, this section describes the underlying mechanical governing equations for a single conducting shielding component, formulated irrespective of the total number $N^{C}$ of shielding components.

The high-dimensional axisymmetric augmented weak form of the mechanical problem is formulated over a higher-dimensional space $\Omega_{p}^{C} \times \Omega_{q}^{u},{ }^{4}$ where $\Omega_{q}^{u}=\Omega_{\omega}^{u} \times \Omega_{\gamma}^{u} \times \Omega_{B_{0}}^{u}$ (the upper index $(\cdot)^{u}$ is used to emphasise the mechanical parametric domain) as: Find $\boldsymbol{u}\left(r, z, \omega, \gamma, B_{0}\right) \in$ $\mathcal{Y}\left(\boldsymbol{u}_{D}\right)$ such that ${ }^{5}$

$$
\mathcal{W}_{K}^{u}(\boldsymbol{u}, \delta \boldsymbol{u})+i \mathcal{W}_{C}^{u}(\boldsymbol{u}, \delta \boldsymbol{u})-\mathcal{W}_{M}^{u}(\boldsymbol{u}, \delta \boldsymbol{u})=-\mathcal{S}^{u}\left(\mathcal{A}_{\phi}, \delta \boldsymbol{u}\right) \quad \forall \delta \boldsymbol{u} \in \mathcal{Y}(\mathbf{0}),
$$

with

$$
\begin{aligned}
\mathcal{W}_{K}^{u}(\boldsymbol{a}, \boldsymbol{b}) & :=\int_{\Omega_{B_{0}}^{u}} \int_{\Omega_{\gamma}^{u}} \int_{\Omega_{\omega}^{u}} W_{K}^{u}(\boldsymbol{a}, \boldsymbol{b}) \mathrm{d} \omega \mathrm{d} \gamma \mathrm{d} B_{0}, \\
\mathcal{W}_{C}^{u}(\boldsymbol{a}, \boldsymbol{b}) & :=\int_{\Omega_{B_{0}}^{u}} \int_{\Omega_{\gamma}^{u}} \int_{\Omega_{\omega}^{u}} \omega^{2} W_{C}^{u}(\boldsymbol{a}, \boldsymbol{b}) \mathrm{d} \omega \mathrm{d} \gamma \mathrm{d} B_{0}, \\
\mathcal{W}_{M}^{u}(\boldsymbol{a}, \boldsymbol{b}) & :=\int_{\Omega_{B_{0}}^{u}} \int_{\Omega_{\gamma}^{u}} \int_{\Omega_{\omega}^{u}} \omega^{2} W_{M}^{u}(\boldsymbol{a}, \boldsymbol{b}) \mathrm{d} \omega \mathrm{d} \gamma \mathrm{d} B_{0}, \\
\mathcal{S}^{u}(a, \boldsymbol{b}) & :=\int_{\Omega_{B_{0}}^{u}} \alpha_{B_{0}} \int_{\Omega_{\gamma}^{u}} \int_{\Omega_{\omega}^{u}} W_{K}^{u A}(a, \boldsymbol{b}) \mathrm{d} \omega \mathrm{d} \gamma \mathrm{d} B_{0},
\end{aligned}
$$

\footnotetext{
${ }^{3}$ The formulation presented in this paper is an extension of the previously developed frequency-based PGD formulation [35], where now the problem is integrated in a higher-dimensional space $\Omega_{p} \times \Omega_{q}$.

${ }^{4}$ Only the conducting spatial domain $\Omega_{p}^{C}$ is considered for the mechanical problem, instead of the entire spatial domain $\Omega_{p}$ used for electromagnetics.

${ }^{5}$ Note that in a small departure from the notation used in [35], where a monolithic solver was developed, the electromagnetic input term is here considered as a source term $\mathcal{S}^{u}\left(\mathcal{A}_{\phi}, \delta \boldsymbol{u}\right)$ on the right-hand side.
} 


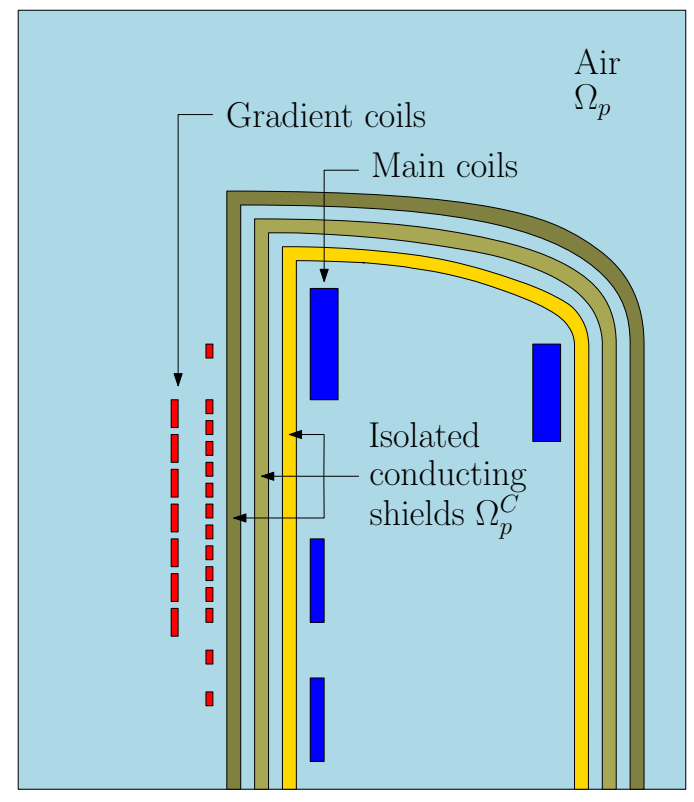

Figure 4: Typical axisymmetric MRI scanner geometry; presence of multiple $\left(N^{C}=3\right)$ isolated conducting components $\Omega_{p}^{C}$ embedded in a truncated non-conducting domain $\Omega_{p}$.

and

$$
\mathcal{Y}\left(\boldsymbol{u}_{D}\right):=\left\{\boldsymbol{u}: \boldsymbol{u} \in\left(H^{1}\left(\Omega_{p}^{C} \times \Omega_{\omega}^{u} \times \Omega_{\gamma}^{u} \times \Omega_{B_{0}}^{u}\right)\right)^{2}, \boldsymbol{u}=\boldsymbol{u}_{D} \text { on } \partial \Omega_{p, D}^{C} \times \Omega_{\omega}^{u} \times \Omega_{\gamma}^{u} \times \Omega_{B_{0}}^{u}\right\}
$$

Note that no direct dependency on the electric conductivity $\gamma$ can be observed in any of the terms in (20). However, since the magnetic potential is $\gamma$-dependent, namely $\mathcal{A}_{\phi}=\mathcal{A}_{\phi}(r, z, \omega, \gamma)$, integration over $\Omega_{\gamma}^{u}$ is required in the above definitions in order to accommodate the right-hand side term $\mathcal{S}^{u}\left(\mathcal{A}_{\phi}, \delta \mathfrak{u}\right)$ in $(20 \mathrm{~d})$.

\section{4. $P G D$ formulation for electromagnetics}

The PGD method is applied to the AC electromagnetic problem (16) by approximating the scalar potential field $\mathcal{A}_{\phi}$ with a separable expression. The general high-dimensional PGD definition for a vector field $\boldsymbol{q}$, see (14), is particularised for the electromagnetic scalar potential $\mathcal{A}_{\phi}$ as

$$
\mathcal{A}_{\phi}(r, z, \omega, \gamma) \approx \mathcal{A}_{\phi}^{N_{A}}(r, z, \omega, \gamma):=\sum_{n=1}^{N_{A}} \beta^{n} F_{\mathfrak{A}_{\phi}}^{n}(r, z) G_{\mathfrak{A}_{\phi}}^{n}(\omega) H_{\mathfrak{A}_{\phi}}^{n}(\gamma),
$$

where $N_{A}$ is the total number of electromagnetic modes computed, the spatial modes $\mathcal{F}^{n}(r, z)$ appearing in (14) reduce to $F_{\mathcal{A}_{\phi}}^{n}(r, z)^{6}$ and the parametric modes $\mathcal{G}_{1}^{n}\left(w_{1}\right)=G_{\mathfrak{A}_{\phi}}^{n}(\omega)$ and $\mathcal{G}_{2}^{n}\left(w_{2}\right)=$ $H_{\mathfrak{A}_{\phi}}^{n}(\gamma)$. The $n^{\text {th }}$ accumulated solution is then

$$
\begin{aligned}
\mathcal{A}_{\phi}^{n}(r, z, \omega, \gamma) & =\sum_{m=1}^{n-1} \beta^{m} F_{\mathfrak{A}_{\phi}}^{m}(r, z) G_{\mathfrak{A}_{\phi}}^{m}(\omega) H_{\mathfrak{A}_{\phi}}^{m}(\gamma)+f_{\mathfrak{A}_{\phi}}(r, z) g_{\mathfrak{A}_{\phi}}(\omega) h_{\mathfrak{A}_{\phi}}(\gamma) \\
& =\mathcal{A}_{\phi}^{n-1}(r, z, \omega, \gamma)+f_{\mathfrak{A}_{\phi}}(r, z) g_{\mathfrak{A}_{\phi}}(\omega) h_{\mathfrak{A}_{\phi}}(\gamma),
\end{aligned}
$$

where, as mentioned in Section 3.1, the uppercase and lowercase fonts indicate converged and non-converged quantities in the process of a fixed-point ADS algorithm. A suitable (compatible)

\footnotetext{
${ }^{6}$ Note that the general separable expression for a vector field $\boldsymbol{q}(14)$ is reduced to a scalar field $\mathcal{A}_{\phi}$ and thus, the vectorial function $\mathcal{F}^{n}(r, z)$ becomes the scalar function $F_{\mathfrak{A}_{\phi}}^{n}(r, z)$.
} 
test function can be introduced as

$$
\delta \mathcal{A}_{\phi}=\delta f_{\mathfrak{A}_{\phi}} g_{\mathfrak{A}_{\phi}} h_{\mathcal{A}_{\phi}}+f_{\mathfrak{A}_{\phi}} \delta g_{\mathfrak{A}_{\phi}} h_{\mathfrak{A}_{\phi}}+f_{\mathfrak{A}_{\phi}} g_{\mathfrak{A}_{\phi}} \delta h_{\mathfrak{A}_{\phi}} .
$$

The two expressions (23) and (24) can be now substituted into the electromagnetic weak form (16), allowing the problem to be formulated as: Find $\left(f_{\mathfrak{A}_{\phi}}, g_{\mathfrak{A}_{\phi}}, h_{\mathfrak{A}_{\phi}}\right) \in X(0) \times Z\left(\Omega_{\omega}^{A}\right) \times$ $Z\left(\Omega_{\gamma}^{A}\right)$ such that

$$
\begin{aligned}
\mathcal{R}_{f_{\mathfrak{A}_{\phi}}}\left(\delta f_{\mathfrak{A}_{\phi}} ; \mathcal{A}_{\phi}^{n-1}, f_{\mathfrak{A}_{\phi}}, g_{\mathfrak{A}_{\phi}}, h_{\mathfrak{A}_{\phi}}\right) & +\mathcal{R}_{g_{\mathfrak{A}_{\phi}}}\left(\delta g_{\mathfrak{A}_{\phi}} ; \mathcal{A}_{\phi}^{n-1}, f_{\mathfrak{A}_{\phi}}, g_{\mathfrak{A}_{\phi}}, h_{\mathfrak{A}_{\phi}}\right) \\
& +\mathcal{R}_{h_{\mathfrak{A}_{\phi}}}\left(\delta h_{\mathfrak{A}_{\phi}} ; \mathcal{A}_{\phi}^{n-1}, f_{\mathfrak{A}_{\phi}}, g_{\mathfrak{A}_{\phi}}, h_{\mathfrak{A}_{\phi}}\right)=0,
\end{aligned}
$$

$\forall\left(\delta f_{\mathfrak{A}_{\phi}}, \delta g_{\mathfrak{A}_{\phi}}, \delta h_{\mathfrak{A}_{\phi}}\right) \in X(0) \times Z\left(\Omega_{\omega}^{A}\right) \times Z\left(\Omega_{\gamma}^{A}\right)$, where $Z(\Omega)=L^{2}(\Omega)$ and the three electromagnetic residual forms are defined as

$$
\begin{aligned}
& \mathcal{R}_{f_{\mathfrak{A}_{\phi}}}\left(\delta f_{\mathcal{A}_{\phi}} ; \mathcal{A}_{\phi}^{n-1}, f_{\mathcal{A}_{\phi}}, g_{\mathfrak{A}_{\phi}}, h_{\mathfrak{A}_{\phi}}\right):=\mathcal{W}_{K}^{A}\left(f_{\mathfrak{A}_{\phi}} g_{\mathfrak{A}_{\phi}} h_{\mathcal{A}_{\phi}}, \delta f_{\mathfrak{A}_{\phi}} g_{\mathfrak{A}_{\phi}} h_{\mathfrak{A}_{\phi}}\right) \\
& +\mathrm{i} \mathcal{W}_{C}^{A}\left(f_{\mathfrak{A}_{\phi}} g_{\mathfrak{A}_{\phi}} h_{\mathfrak{A}_{\phi}}, \delta f_{\mathfrak{A}_{\phi}} g_{\mathfrak{A}_{\phi}} h_{\mathfrak{A}_{\phi}}\right) \\
& -\mathcal{S}^{A}\left(\delta f_{\mathfrak{A}_{\phi}} g_{\mathfrak{A}_{\phi}} h_{\mathfrak{A}_{\phi}}\right)+\mathcal{W}_{K}^{A}\left(\mathcal{A}_{\phi}^{n-1}, \delta f_{\mathfrak{A}_{\phi}} g_{\mathfrak{A}_{\phi}} h_{\mathfrak{A}_{\phi}}\right) \\
& +\mathrm{i} \mathcal{W}_{C}^{A}\left(\mathcal{A}_{\phi}^{n-1}, \delta f_{\mathcal{A}_{\phi}} g_{\mathfrak{A}_{\phi}} h_{\mathfrak{A}_{\phi}}\right) \text {, } \\
& \mathcal{R}_{g_{\mathfrak{A}_{\phi}}}\left(\delta g_{\mathfrak{A}_{\phi}} ; \mathcal{A}_{\phi}^{n-1}, f_{\mathfrak{A}_{\phi}}, g_{\mathfrak{A}_{\phi}}, h_{\mathfrak{A}_{\phi}}\right):=\mathcal{W}_{K}^{A}\left(f_{\mathfrak{A}_{\phi}} g_{\mathfrak{A}_{\phi}} h_{\mathfrak{A}_{\phi}}, f_{\mathfrak{A}_{\phi}} \delta g_{\mathfrak{A}_{\phi}} h_{\mathfrak{A}_{\phi}}\right) \\
& +\mathrm{i} \mathcal{W}_{C}^{A}\left(f_{\mathfrak{A}_{\phi}} g_{\mathfrak{A}_{\phi}} h_{\mathfrak{A}_{\phi}}, f_{\mathfrak{A}_{\phi}} \delta g_{\mathfrak{A}_{\phi}} h_{\mathfrak{A}_{\phi}}\right) \\
& -\mathcal{S}^{A}\left(f_{\mathfrak{A}_{\phi}} \delta g_{\mathfrak{A}_{\phi}} h_{\mathfrak{A}_{\phi}}\right)+\mathcal{W}_{K}^{A}\left(\mathfrak{A}_{\phi}^{n-1}, f_{\mathfrak{A}_{\phi}} \delta g_{\mathfrak{A}_{\phi}} h_{\mathfrak{A}_{\phi}}\right) \\
& +\mathrm{i} \mathcal{W}_{C}^{A}\left(\mathcal{A}_{\phi}^{n-1}, f_{\mathfrak{A}_{\phi}} \delta g_{\mathfrak{A}_{\phi}} h_{\mathcal{A}_{\phi}}\right) \text {, } \\
& \mathcal{R}_{h_{\mathfrak{A}_{\phi}}}\left(\delta h_{\mathcal{A}_{\phi}} ; \mathcal{A}_{\phi}^{n-1}, f_{\mathfrak{A}_{\phi}}, g_{\mathfrak{A}_{\phi}}, h_{\mathcal{A}_{\phi}}\right):=\mathcal{W}_{K}^{A}\left(f_{\mathfrak{A}_{\phi}} g_{\mathcal{A}_{\phi}} h_{\mathcal{A}_{\phi}}, f_{\mathfrak{A}_{\phi}} g_{\mathfrak{A}_{\phi}} \delta h_{\mathfrak{A}_{\phi}}\right) \\
& +\mathrm{i} \mathcal{W}_{C}^{A}\left(f_{\mathfrak{A}_{\phi}} g_{\mathfrak{A}_{\phi}} h_{\mathfrak{A}_{\phi}}, f_{\mathfrak{A}_{\phi}} g_{\mathfrak{A}_{\phi}} \delta h_{\mathfrak{A}_{\phi}}\right) \\
& -\mathcal{S}^{A}\left(f_{\mathfrak{A}_{\phi}} g_{\mathfrak{A}_{\phi}} \delta h_{\mathfrak{A}_{\phi}}\right)+\mathcal{W}_{K}^{A}\left(\mathcal{A}_{\phi}^{n-1}, f_{\mathfrak{A}_{\phi}} g_{\mathfrak{A}_{\phi}} \delta h_{\mathfrak{A}_{\phi}}\right) \\
& +\mathrm{i} \mathcal{W}_{C}^{A}\left(\mathcal{A}_{\phi}^{n-1}, f_{\mathfrak{A}_{\phi}} g_{\mathfrak{A}_{\phi}} \delta h_{\mathcal{A}_{\phi}}\right) \text {, }
\end{aligned}
$$

Regarding the implementation of the electromagnetic problem (25), this can be formulated using the concept of directional derivatives [40], where the problem is solved by incrementally updating the solution fields $f_{\mathfrak{A}_{\phi}}, g_{\mathfrak{A}_{\phi}}$ and $h_{\mathfrak{A}_{\phi}}$. As mentioned in Section 3.1, the solver exploits a fixed-point ADS in order to efficiently converge within every modal component of the PGD solution. The detailed formulation and its implementation is presented in Appendix A.

\section{5. $P G D$ formulation for mechanics}

In a similar manner to electromagnetics, the PGD methodology approximates the displacement field $\mathcal{U}$ using a separable representation. In this case, the generic high-dimensional PGD definition (14) becomes

$$
\boldsymbol{u}\left(r, z, \omega, \gamma, B_{0}\right) \approx \boldsymbol{U}^{N_{u}}\left(r, z, \omega, \gamma, B_{0}\right):=\sum_{n=1}^{N_{u}} \beta^{n} \boldsymbol{F}_{\boldsymbol{u}}^{n}(r, z) G_{\boldsymbol{u}}^{n}(\omega) H_{\boldsymbol{u}}^{n}(\gamma) L_{\boldsymbol{u}}^{n}\left(B_{0}\right),
$$

where $N_{u}$ is the number of mechanical modes computed and the terms appearing in the general PGD definition (14) become $\mathcal{F}^{n}(r, z)=\boldsymbol{F}_{\boldsymbol{u}}^{n}(r, z), \mathcal{G}_{1}^{n}\left(w_{1}\right)=G_{\boldsymbol{u}}^{n}(\omega), \mathcal{G}_{2}^{n}\left(w_{2}\right)=H_{\boldsymbol{u}}^{n}(\gamma)$ and $\mathcal{G}_{3}^{n}\left(w_{3}\right)=L_{\boldsymbol{u}}^{n}\left(B_{0}\right)$. Considering a particular modal component $n$, the accumulated solution field is written as

$$
\begin{aligned}
\boldsymbol{u}^{n}\left(r, z, \omega, \gamma, B_{0}\right) & =\sum_{m=1}^{n-1} \beta^{m} \boldsymbol{F}_{\boldsymbol{u}}^{m}(r, z) G_{\boldsymbol{u}}^{m}(\omega) H_{\boldsymbol{u}}^{m}(\gamma) L_{\boldsymbol{u}}^{m}\left(B_{0}\right)+\boldsymbol{f}_{\boldsymbol{u}}(r, z) g_{\boldsymbol{u}}(\omega) h_{\boldsymbol{u}}(\gamma) l_{\boldsymbol{u}}\left(B_{0}\right) \\
& =\boldsymbol{u}^{n-1}\left(r, z, \omega, \gamma, B_{0}\right)+\boldsymbol{f}_{\boldsymbol{u}}(r, z) g_{\boldsymbol{u}}(\omega) h_{\boldsymbol{u}}(\gamma) l_{\boldsymbol{u}}\left(B_{0}\right)
\end{aligned}
$$


where a suitable (compatible) test function can be introduced as

$$
\delta \boldsymbol{u}=\delta \boldsymbol{f}_{\boldsymbol{u}} g_{\boldsymbol{u}} h_{\boldsymbol{u}} l_{\boldsymbol{u}}+\boldsymbol{f}_{\boldsymbol{u}} \delta g_{\boldsymbol{u}} h_{\boldsymbol{u}} l_{\boldsymbol{u}}+\boldsymbol{f}_{\boldsymbol{u}} g_{\boldsymbol{u}} \delta h_{\boldsymbol{u}} l_{\boldsymbol{u}}+\boldsymbol{f}_{\boldsymbol{u}} g_{\boldsymbol{u}} h_{\boldsymbol{u}} \delta l_{\boldsymbol{u}}
$$

These two expressions (28) and (29) can be substituted into the mechanical augmented weak form (19) together with the electromagnetic PGD definition (22), allowing the problem to be formulated as: Find $\left(\boldsymbol{f}_{\boldsymbol{u}}, g_{\boldsymbol{u}}, h_{\boldsymbol{u}}, l_{\boldsymbol{u}}\right) \in Y\left(\boldsymbol{u}_{D}\right) \times Z\left(\Omega_{\omega}^{u}\right) \times Z\left(\Omega_{\gamma}^{u}\right) \times Z\left(\Omega_{B_{0}}^{u}\right)$ such that

$$
\begin{aligned}
& \mathcal{R}_{\boldsymbol{f}_{\boldsymbol{u}}}\left(\delta \boldsymbol{f}_{\boldsymbol{u}} ; \boldsymbol{u}^{n-1}, \boldsymbol{f}_{\boldsymbol{u}}, g_{\boldsymbol{u}}, h_{\boldsymbol{u}}, l_{\boldsymbol{u}}\right)+\mathcal{R}_{g_{\boldsymbol{u}}}\left(\delta g_{\boldsymbol{u}} ; \boldsymbol{u}^{n-1}, \boldsymbol{f}_{\boldsymbol{u}}, g_{\boldsymbol{u}}, h_{\boldsymbol{u}}, l_{\boldsymbol{u}}\right) \\
+ & \mathcal{R}_{h_{\boldsymbol{u}}}\left(\delta h_{\boldsymbol{u}} ; \boldsymbol{u}^{n-1}, \boldsymbol{f}_{\boldsymbol{u}}, g_{\boldsymbol{u}}, h_{\boldsymbol{u}}, l_{\boldsymbol{u}}\right)+\mathcal{R}_{l_{\boldsymbol{u}}}\left(\delta l_{\boldsymbol{u}} ; \boldsymbol{u}^{n-1}, \boldsymbol{f}_{\boldsymbol{u}}, g_{\boldsymbol{u}}, h_{\boldsymbol{u}}, l_{\boldsymbol{u}}\right)=0
\end{aligned}
$$

$\forall\left(\delta \boldsymbol{f}_{\boldsymbol{u}}, \delta g_{\boldsymbol{u}}, \delta h_{\boldsymbol{u}}, \delta l_{\boldsymbol{u}}\right) \in Y(0) \times Z\left(\Omega_{\omega}^{u}\right) \times Z\left(\Omega_{\gamma}^{u}\right) \times Z\left(\Omega_{B_{0}}^{u}\right)$ and the four mechanical residual forms are defined as

$$
\begin{aligned}
& \mathcal{R}_{\boldsymbol{f}_{\boldsymbol{u}}}\left(\delta \boldsymbol{f}_{\boldsymbol{u}} ; \boldsymbol{u}^{n-1}, \boldsymbol{f}_{\boldsymbol{u}}, g_{\boldsymbol{u}}, h_{\boldsymbol{u}}, l_{\boldsymbol{u}}\right):=\mathcal{W}_{K}^{u}\left(\boldsymbol{f}_{\boldsymbol{u}} g_{\boldsymbol{u}} h_{\boldsymbol{u}} l_{\boldsymbol{u}}, \delta \boldsymbol{f}_{\boldsymbol{u}} g_{\boldsymbol{u}} h_{\boldsymbol{u}} l_{\boldsymbol{u}}\right) \\
& +\mathrm{i} \mathcal{W}_{C}^{u}\left(\boldsymbol{f}_{\boldsymbol{u}} g_{\boldsymbol{u}} h_{\boldsymbol{u}} l_{\boldsymbol{u}}, \delta \boldsymbol{f}_{\boldsymbol{u}} g_{\boldsymbol{u}} h_{\boldsymbol{u}} l_{\boldsymbol{u}}\right) \\
& -\mathcal{W}_{M}^{u}\left(\boldsymbol{f}_{\boldsymbol{u}} g_{\boldsymbol{u}} h_{\boldsymbol{u}} l_{\boldsymbol{u}}, \delta \boldsymbol{f}_{\boldsymbol{u}} g_{\boldsymbol{u}} h_{\boldsymbol{u}} l_{\boldsymbol{u}}\right) \\
& +\mathcal{S}^{u}\left(\mathcal{A}_{\phi}^{N_{A}}, \delta \boldsymbol{f}_{\boldsymbol{u}} g_{\boldsymbol{u}} h_{\boldsymbol{u}} l_{\boldsymbol{u}}\right)+\mathcal{W}_{K}^{u}\left(\boldsymbol{u}^{n-1}, \delta \boldsymbol{f}_{\boldsymbol{u}} g_{\boldsymbol{u}} h_{\boldsymbol{u}} l_{\boldsymbol{u}}\right) \\
& +\mathrm{i} \mathcal{W}_{C}^{u}\left(\boldsymbol{u}^{n-1}, \delta \boldsymbol{f}_{\boldsymbol{u}} g_{\boldsymbol{u}} h_{\boldsymbol{u}} l_{\boldsymbol{u}}\right)-\mathcal{W}_{M}^{u}\left(\boldsymbol{u}^{n-1}, \delta \boldsymbol{f}_{\boldsymbol{u}} g_{\boldsymbol{u}} h_{\boldsymbol{u}} l_{\boldsymbol{u}}\right), \\
& \mathcal{R}_{g_{\boldsymbol{u}}}\left(\delta g_{\boldsymbol{u}} ; \boldsymbol{u}^{n-1}, \boldsymbol{f}_{\boldsymbol{u}}, g_{\boldsymbol{u}}, h_{\boldsymbol{u}}, l_{\boldsymbol{u}}\right):=\mathcal{W}_{K}^{u}\left(\boldsymbol{f}_{\boldsymbol{u}} g_{\boldsymbol{u}} h_{\boldsymbol{u}} l_{\boldsymbol{u}}, \boldsymbol{f}_{\boldsymbol{u}} \delta g_{\boldsymbol{u}} h_{\boldsymbol{u}} l_{\boldsymbol{u}}\right) \\
& +\mathrm{i} \mathcal{W}_{C}^{u}\left(\boldsymbol{f}_{\boldsymbol{u}} g_{\boldsymbol{u}} h_{\boldsymbol{u}} l_{\boldsymbol{u}}, \boldsymbol{f}_{\boldsymbol{u}} \delta g_{\boldsymbol{u}} h_{\boldsymbol{u}} l_{\boldsymbol{u}}\right) \\
& -\mathcal{W}_{M}^{u}\left(\boldsymbol{f}_{\boldsymbol{u}} g_{\boldsymbol{u}} h_{\boldsymbol{u}} l_{\boldsymbol{u}}, \boldsymbol{f}_{\boldsymbol{u}} \delta g_{\boldsymbol{u}} h_{\boldsymbol{u}} l_{\boldsymbol{u}}\right) \\
& +\mathcal{S}^{u}\left(\mathcal{A}_{\phi}^{N_{A}}, \boldsymbol{f}_{\boldsymbol{u}} \delta g_{\boldsymbol{u}} h_{\boldsymbol{u}} l_{\boldsymbol{u}}\right)+\mathcal{W}_{K}^{u}\left(\boldsymbol{u}^{n-1}, \boldsymbol{f}_{\boldsymbol{u}} \delta g_{\boldsymbol{u}} h_{\boldsymbol{u}} l_{\boldsymbol{u}}\right) \\
& +\mathrm{i} \mathcal{W}_{C}^{u}\left(\boldsymbol{u}^{n-1}, \boldsymbol{f}_{\boldsymbol{u}} \delta g_{\boldsymbol{u}} h_{\boldsymbol{u}} l_{\boldsymbol{u}}\right)-\mathcal{W}_{M}^{u}\left(\boldsymbol{u}^{n-1}, \boldsymbol{f}_{\boldsymbol{u}} \delta g_{\boldsymbol{u}} h_{\boldsymbol{u}} l_{\boldsymbol{u}}\right), \\
& \mathcal{R}_{h_{\boldsymbol{u}}}\left(\delta h_{\boldsymbol{u}} ; \boldsymbol{u}^{n-1}, \boldsymbol{f}_{\boldsymbol{u}}, g_{\boldsymbol{u}}, h_{\boldsymbol{u}}, l_{\boldsymbol{u}}\right):=\mathcal{W}_{K}^{u}\left(\boldsymbol{f}_{\boldsymbol{u}} g_{\boldsymbol{u}} h_{\boldsymbol{u}} l_{\boldsymbol{u}}, \boldsymbol{f}_{\boldsymbol{u}} g_{\boldsymbol{u}} \delta h_{\boldsymbol{u}} l_{\boldsymbol{u}}\right) \\
& +\mathrm{i} \mathcal{W}_{C}^{u}\left(\boldsymbol{f}_{\boldsymbol{u}} g_{\boldsymbol{u}} h_{\boldsymbol{u}} l_{\boldsymbol{u}}, \boldsymbol{f}_{\boldsymbol{u}} g_{\boldsymbol{u}} \delta h_{\boldsymbol{u}} l_{\boldsymbol{u}}\right) \\
& -\mathcal{W}_{M}^{u}\left(\boldsymbol{f}_{\boldsymbol{u}} g_{\boldsymbol{u}} h_{\boldsymbol{u}} l_{\boldsymbol{u}}, \boldsymbol{f}_{\boldsymbol{u}} g_{\boldsymbol{u}} \delta h_{\boldsymbol{u}} l_{\boldsymbol{u}}\right) \\
& +\mathcal{S}^{u}\left(\mathcal{A}_{\phi}^{N_{A}}, \boldsymbol{f}_{\boldsymbol{u}} g_{\boldsymbol{u}} \delta h_{\boldsymbol{u}} l_{\boldsymbol{u}}\right)+\mathcal{W}_{K}^{u}\left(\boldsymbol{u}^{n-1}, \boldsymbol{f}_{\boldsymbol{u}} g_{\boldsymbol{u}} \delta h_{\boldsymbol{u}} l_{\boldsymbol{u}}\right) \\
& +\mathrm{i} \mathcal{W}_{C}^{u}\left(\boldsymbol{u}^{n-1}, \boldsymbol{f}_{\boldsymbol{u}} g_{\boldsymbol{u}} \delta h_{\boldsymbol{u}} l_{\boldsymbol{u}}\right)-\mathcal{W}_{M}^{u}\left(\boldsymbol{u}^{n-1}, \boldsymbol{f}_{\boldsymbol{u}} g_{\boldsymbol{u}} \delta h_{\boldsymbol{u}} l_{\boldsymbol{u}}\right), \\
& \mathcal{R}_{l_{\boldsymbol{u}}}\left(\delta l_{\boldsymbol{u}} ; \boldsymbol{u}^{n-1}, \boldsymbol{f}_{\boldsymbol{u}}, g_{\boldsymbol{u}}, h_{\boldsymbol{u}}, l_{\boldsymbol{u}}\right):=\mathcal{W}_{K}^{u}\left(\boldsymbol{f}_{\boldsymbol{u}} g_{\boldsymbol{u}} h_{\boldsymbol{u}} l_{\boldsymbol{u}}, \boldsymbol{f}_{\boldsymbol{u}} g_{\boldsymbol{u}} h_{\boldsymbol{u}} \delta l_{\boldsymbol{u}}\right) \\
& +\mathrm{i} \mathcal{W}_{C}^{u}\left(\boldsymbol{f}_{\boldsymbol{u}} g_{\boldsymbol{u}} h_{\boldsymbol{u}} l_{\boldsymbol{u}}, \boldsymbol{f}_{\boldsymbol{u}} g_{\boldsymbol{u}} h_{\boldsymbol{u}} \delta l_{\boldsymbol{u}}\right) \\
& -\mathcal{W}_{M}^{u}\left(\boldsymbol{f}_{\boldsymbol{u}} g_{\boldsymbol{u}} h_{\boldsymbol{u}} l_{\boldsymbol{u}}, \boldsymbol{f}_{\boldsymbol{u}} g_{\boldsymbol{u}} h_{\boldsymbol{u}} \delta l_{\boldsymbol{u}}\right) \\
& +\mathcal{S}^{u}\left(\mathcal{A}_{\phi}^{N_{A}}, \boldsymbol{f}_{\boldsymbol{u}} g_{\boldsymbol{u}} h_{\boldsymbol{u}} \delta l_{\boldsymbol{u}}\right)+\mathcal{W}_{K}^{u}\left(\boldsymbol{u}^{n-1}, \boldsymbol{f}_{\boldsymbol{u}} g_{\boldsymbol{u}} h_{\boldsymbol{u}} \delta l_{\boldsymbol{u}}\right) \\
& +\mathrm{i} \mathcal{W}_{C}^{u}\left(\boldsymbol{u}^{n-1}, \boldsymbol{f}_{\boldsymbol{u}} g_{\boldsymbol{u}} h_{\boldsymbol{u}} \delta l_{\boldsymbol{u}}\right)-\mathcal{W}_{M}^{u}\left(\boldsymbol{u}^{n-1}, \boldsymbol{f}_{\boldsymbol{u}} g_{\boldsymbol{u}} h_{\boldsymbol{u}} \delta l_{\boldsymbol{u}}\right) .
\end{aligned}
$$

The mechanical problem (30) is solved following an analogous procedure to that for electromagnetics, see (25), where further details can be found in Appendix A. Note that in the case of the mechanical problem, input information arising from the electromagnetic problem is needed, see the right-hand side in (19), which leads to the introductions of so-called mortar integral due to the possibility of considering different levels of discretisation in the parametric domains, as shown in Section 3.2. The numerical treatment of these mortar integrals is discussed and formulated in Appendix B.

\subsection{Staggered $P G D$ solver}

The PGD solver described above exploits the staggered nature of the coupled magnetomechanical problem (6) and (10), by solving the coupled magneto-mechanical problem in a 
sequential manner, as the workflow describes in Figure 5 where, the high-dimensional AC electromagnetic problem (25) is solved first in order to obtain a separable representation for the scalar potential $\mathcal{A}_{\phi}^{N_{A}}$. Subsequently, the high-dimensional AC mechanical problem (30) is solved for each mechanical component, until the complete mechanical response $\boldsymbol{u}^{N_{u}}$ is obtained. The offline stage of the PGD method ends when the separable expressions for both physics $\mathcal{A}_{\phi}^{N_{A}}$ and $\boldsymbol{u}^{N_{u}}$ are obtained and stored for future online postprocessing.

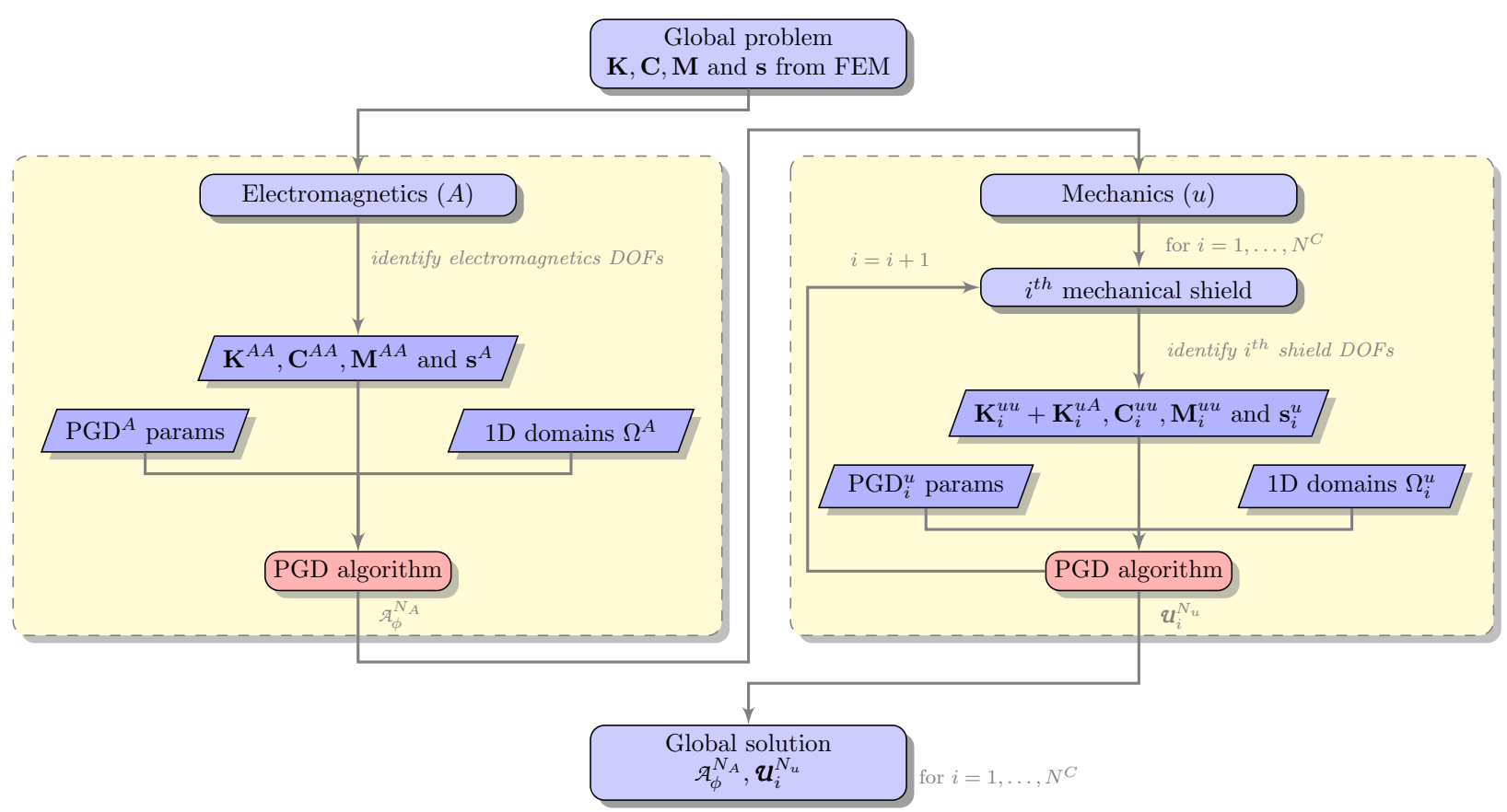

Figure 5: Flow chart algorithm; description of the staggered PGD nature. The continuous and discrete formulations are detailed in Appendix A.

\section{Electromagnetic-Proper Orthogonal Decomposition (EM-POD) methodology}

This section presents an alternative a posteriori ROM technique which, by also exploiting the staggered nature of the underlying coupled equations, combines the POD method for electromagnetics and the full order model for mechanics (referred to as EM-POD hereafter) ${ }^{7}$. The methodology, in addition to be used for benchmarking purposes, will help draw some meaningful conclusions in terms of computational effort when comparing it against the staggered PGD method. Whilst in [36], this EM-POD methodology was applied to three-dimensional configurations, this is here suitably adapted to account for axisymmetric configurations.

The fundamental idea behind the EM-POD methodology relies on the application of the POD method to the electromagnetic problem in order to obtain an approximation $\mathcal{A}_{\phi}^{P O D}(r, z, \omega, \gamma)$ to $\mathcal{A}_{\phi}(r, z, \omega, \gamma)$ and then use this as an input source to the mechanical full order problem to compute $\boldsymbol{u}\left(r, z, \omega, \gamma, B_{0}\right) .{ }^{8}$ Before that, the problem is first discretised in space using a typical FEM expansion as

$$
\mathcal{A}_{\phi}(r, z, \omega, \gamma) \approx \sum_{i=1}^{Q_{A}} N_{i}(r, z) \mathcal{A}_{i}(\omega, \gamma), \quad \boldsymbol{u}\left(r, z, \omega, \gamma, B_{0}\right) \approx \sum_{i=1}^{Q_{u}} N_{i}(r, z) \mathcal{U}_{i}\left(\omega, \gamma, B_{0}\right),
$$

\footnotetext{
${ }^{7}$ Note that this methodology was recently developed in [36] under the name "combined POD-full order model" approach.

${ }^{8}$ For a more generic description of the POD method applied to an arbitrary number of parameters, namely $\mathcal{A}_{\phi}\left(r, z, w_{1}, \ldots, w_{d}\right)$ and $\boldsymbol{u}\left(r, z, w_{1}, \ldots, w_{d}\right)$, the reader is referred to [36].
} 
where $Q_{A}$ and $Q_{u}$ are the number of electromagnetic and mechanical degrees of freedom, respectively, and $N_{i}$ denote typical hierarchic $H^{1}$ conforming basis functions, which can be rewritten in matrix notation as

$$
\mathcal{A}_{\phi}(r, z, \omega, \gamma) \approx \mathbf{N}^{\mathrm{A}}(r, z) \mathcal{A}(\omega, \gamma), \quad \boldsymbol{u}\left(r, z, \omega, \gamma, B_{0}\right) \approx \mathbf{N}^{\mathrm{u}}(r, z) \mathcal{U}\left(\omega, \gamma, B_{0}\right)
$$

where we have defined the discrete vectors of coefficients $\mathcal{A}(\omega, \gamma), \mathcal{U}\left(\omega, \gamma, B_{0}\right)$ and the matrices $\mathbf{N}^{\mathrm{A}}(r, z)$ and $\mathbf{N}^{\mathrm{u}}(r, z)$ are defined as

$$
\begin{aligned}
\mathbf{N}^{\mathrm{A}}(r, z) & :=\left[N_{1} \ldots N_{Q_{A}}\right] \\
\mathbf{N}^{\mathrm{u}}(r, z):=\left[\mathbf{n}_{1}^{\mathrm{u}} \ldots \mathbf{n}_{Q_{u}}^{\mathrm{u}}\right] & =\left[\begin{array}{ccccc}
N_{1} & 0 & \ldots & N_{Q_{u}} & 0 \\
0 & N_{1} & \ldots & 0 & N_{Q_{u}}
\end{array}\right] .
\end{aligned}
$$

At this point, POD is applied to approximate $\mathcal{A}(\omega, \gamma)$, which is carried out by means of the method of snapshots [41, 42]. First, the vector $\mathbf{w}$ is defined as $\mathbf{w}:=\left(\mathbf{w}_{1} \ldots \mathbf{w}_{N_{s}}\right)$, which contains $N_{s}$ parameter sets (combinations of different parameter values $\omega$ and $\gamma$ ). Then, a matrix of $N_{s}$ snapshots ${ }^{9} \mathbf{D} \in \mathbb{C}^{Q_{A} \times N_{s}}$ is built as

$$
\mathbf{D}:=\left[\mathcal{A}\left(\mathbf{w}_{1}\right) \ldots \mathcal{A}\left(\mathbf{w}_{N_{s}}\right)\right]
$$

where $\mathcal{A}\left(\mathbf{w}_{j}\right)$ is a single snapshot obtained for the parameter set $\mathbf{w}_{j}$. Then, a TSVD [43, 44] is applied in order to obtain a low rank approximation to the snapshot matrix as

$$
\mathbf{D} \approx \mathbf{D}^{M}:=\sum_{k=1}^{M} \mathbf{h}_{k} \sigma_{k} \overline{\mathbf{g}}_{k}^{T}=\mathbf{H}^{M} \boldsymbol{\Sigma}^{M}\left(\overline{\mathbf{G}}^{M}\right)^{T},
$$

where $\mathbf{H}^{M}=\left[\mathbf{h}_{1} \ldots \mathbf{h}_{M}\right] \in \mathbb{C}^{Q_{A} \times M}$ is a matrix containing the first $M$ left singular vectors of $\mathbf{D}$, $\mathbf{G}^{M}=\left[\mathbf{g}_{1} \ldots \mathbf{g}_{M}\right] \in \mathbb{C}^{N_{s} \times M}$ is the matrix of right singular vectors and $\boldsymbol{\Sigma}^{M}=\operatorname{diag}\left(\sigma_{1} \ldots \sigma_{M}\right) \in$ $\mathbb{R}^{M \times M}$ is a diagonal matrix containing the first $M$ singular values, which are sorted in decreasing order. Note that the choice here is to consider the physical space $(r, z)$ and the parametric space $(\omega, \gamma)$ as the two dimensions that have to be separated, so the TSVD can be applied. Otherwise, the high-order version of the TSVD (High-Order Singular Value Decomposition HOSVD $[19,45])$ would be required to separate more than two dimensions, loosing the known optimality of the TSVD [23]. The snapshot calculation and the TSVD constitute the so-called offline stage of the POD algorithm as presented in the left box in Figure 6, where the EM-POD algorithm is summarised using a flow chart. In this particular problem, the affine decomposition structure of the electromagnetic problem (6) is used to perform most of the assembly (the nonparameter dependent assembly) as part of the offline stage ${ }^{10}$, resulting in a much faster online POD stage.

Subsequently, at the online stage, either interpolation based POD (PODI) [46-48] or projection based POD (PODP or POD-Galerkin) [49-51] can be considered in order to obtain $\mathcal{A}(\omega, \gamma)$ for any $\omega$ and $\gamma$. In this case, we explore the use of PODP and $\mathcal{A}(\omega, \gamma)$ is approximated as a linear combination of above left singular vectors as

$$
\mathcal{A}(\omega, \gamma) \approx \mathcal{A}^{\mathrm{POD}}(\omega, \gamma):=\sum_{k=1}^{M} \mathbf{h}_{k} \mathrm{~g}_{k}^{\mathrm{P}}(\omega, \gamma)=\mathbf{H}^{M} \mathbf{g}^{P}(\omega, \gamma)
$$

\footnotetext{
${ }^{9}$ Typically, the snapshots are obtained after solving the so-called full order model.

${ }^{10}$ In most problems, the matrix assembly has to be carried out within the online stage making this POD stage less computationally efficient.
} 
where $\mathbf{h}_{k}$ represents the $k$-th column of the $\mathbf{H}^{M}$ matrix and the parametric mode vector $\mathbf{g}^{P}(\omega, \gamma)$ (of coefficients $\mathrm{g}_{k}^{\mathrm{P}}(\omega, \gamma)$ ) is yet unknown and must be computed for each $\omega$ and $\gamma$. The computation of the unknown vector $\mathbf{g}^{P}(\omega, \gamma)$ is obtained from the solution of the weak form (6) by using a Galerkin approximation with $(33)_{a}$ and (37). This leads to the solution of a reduced system of size $M \times M$ as shown in Figure 6 . This must be applied $N_{o}$ times to obtain the solution for all the parameters sets of interest $\mathbf{w}_{1}, \ldots, \mathbf{w}_{N_{o}}$.

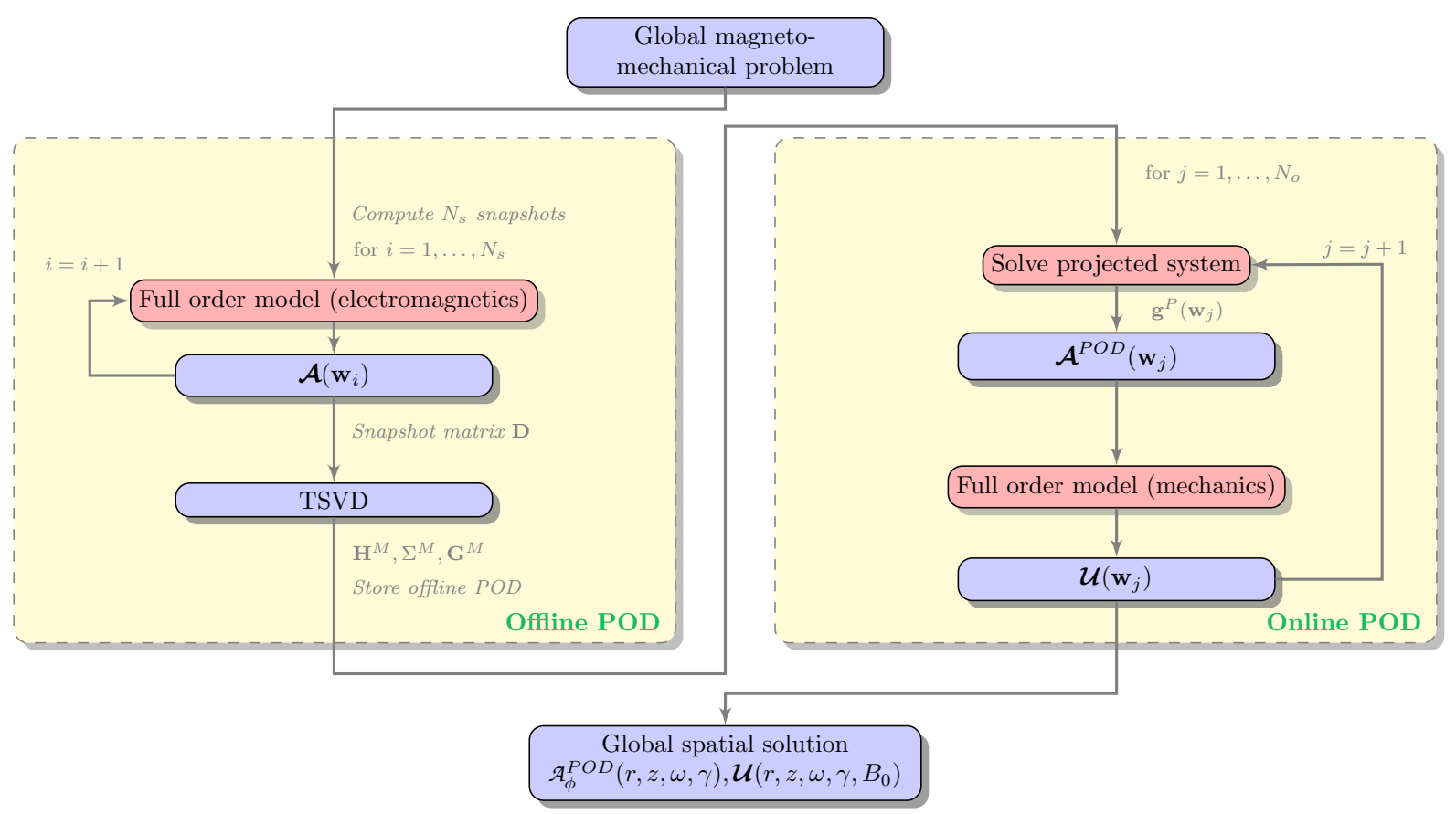

Figure 6: Flow chart algorithm; description of the EM-POD technique.

Once the solution $\mathcal{A}_{\phi}^{P O D}(r, z, \omega, \gamma)$ is obtained, this is used as a source term for the mechanical full order solver [36]. The online stage is graphically presented in the right box of Figure 6. For further details about the EM-POD technique and its performance in the context of three-dimensional configurations, the reader is referred to [36].

\section{Numerical results}

This section includes a series of numerical examples in order to demonstrate the robustness and applicability of the new staggered PGD algorithm derived in Section 3. With this in mind, two different geometries representing two alternative MRI configurations are analysed: first, a simplified geometrical representation followed by a more realistic MRI scanner configuration. One of the aims of the section is to validate the previously described staggered PGD algorithm against the full order (considered as a reference) model. The presence of numerical singularities in the solution fields, due to the existence of resonance, is a challenging localised behaviour (only present in some frequencies of interest) that can be captured by combining the staggered PGD method with an automatic frequency splitting algorithm previously developed by the authors in [35]. As part of the various analyses carried out, integrated quantities of interest such as global dissipated Ohmic current and kinetic energy are displayed. Moreover, the true power of the PGD method is demonstrated by depicting field quantities of interest (i.e. magnetic potential, eddy current distribution) across the entire computational domain, queried in real time.

In addition, the benefits of the new staggered PGD model are also remarked by comparing this new PGD approach against the alternative PGD formulation recently developed by the 
authors in [35], where only the frequency was considered as a parameter of interest and both electromagnetic and mechanical equations were solved simultaneously in a monolithic manner. Finally, the novel PGD staggered model is compared (in terms of accuracy and computational effort) against the alternative EM-POD algorithm included in Section 4, originally developed in [36] for three-dimensions and adapted in this paper for the consideration of axisymmetric scenarios.

\subsection{Test magnet problem}

The first numerical example that is presented in this section depicts a simplification of a realistic MRI scanner configuration. MRI scanners are typically comprised of three main components, namely, main coils responsible for the generation of the static DC field; gradient coils, responsible for the transient $\mathrm{AC}$ fields and radiation shields. Note that the same geometrical representation was already presented in [35], but it is also described here for completeness. The shields correspond to three rectangular (yellow) conducting components embedded in a non-conducing domain (i.e. the air). These are the outer vacuum chamber $\Omega_{O V C}^{C}$, the $77 \mathrm{~K}$ radiation shield $\Omega_{77 K}^{C}$ and the $4 \mathrm{~K}$ helium vessel $\Omega_{4 K}^{C}$. Each mechanical shield has different material and geometrical parameters that cannot be specified due to confidentiality reasons. However, the approximate values are $\mu_{*} \approx 10^{-7} \mathrm{H} / \mathrm{m}, \gamma_{*} \approx 10^{6} \mathrm{~S} / \mathrm{m}, \rho \approx 10^{3} \mathrm{Kg} / \mathrm{m}^{3}, E \approx 10^{9}$ $\mathrm{Pa}$ and $\nu \approx 0.3$. Note that the axisymmetric formulation [13] follows from assuming rotational symmetry of the weak form expressed in 3D cylindrical coordinates and, as such, $r=0$ does not form a boundary of the computational domain $\Omega_{p}$.

\subsubsection{ROM techniques on the electromagnetic problem}

The authors in [36] recently showed that the solution of the mechanical equations does not benefit from using POD, as a similar number of snapshots to that of a full order model was needed in order to accurately capture the location of the resonance (mechanical) singularities. As a result, in this section, we restrict to the un-coupled electromagnetic problem and compare both PGD and EM-POD strategies against the full order model, both in terms of accuracy and computational effort. PGD and EM-POD have been presented in Sections 3 and 4, respectively, and they are implemented herein for the electromagnetic problem of the test magnet geometry, in order to approximate the scalar magnetic potential field $\mathcal{A}_{\phi}=\mathcal{A}_{\phi}(r, z, \omega, \gamma)$ dependent upon the spatial domain $(r, z)$ and two extra parameters, namely, the angular frequency $\omega$ and the electric conductivity $\gamma$.

Regarding PGD, the user-defined parameters are presented in Table 1, where $I_{N}^{A}$ represents the maximum number of modes (note that the superscript $\left(\cdot{ }^{A}\right)$ is used to emphasise the electromagnetic problem), $I_{F P}$ is the maximum number of fixed-point iterations, $t_{N}^{A}$ is the tolerance for the greedy algorithm and $t o l_{F P}$ is the tolerance for the fixed-point algorithm. The parametric domains are defined by a dimensionless mesh size parameter $h$ that is computed as the maximum (FEM) element size divided by a reference size of the domain. For instance, for the frequency domain $\Omega_{\omega}$, the dimensionless mesh size is $h_{\omega}=0.1 / 5000=2 \cdot 10^{-5}$. The remaining user-defined parameters refer to the spatial problem $\Omega_{p}$, where $h_{\Omega_{p}}$ is the mesh size parameter as previously explained, $p$ is the polynomial order used in the FEM discretisation and $\xi$, the dimensionless damping ratio, is used to account both for physical (i.e. classical damping) and numerical regularisation [35].

Regarding EM-POD, see Figure 6, the algorithm is initiated given a series of snapshots computed using the electromagnetics full order model, which in this case consists of a combination of 23 frequencies $f^{\text {snap }}$ and 3 scaling of the electric conductivity $\alpha_{\gamma}^{\text {snap }}$ that make up a total of 69 snapshots $\left(N_{s}=69\right)$. Note that an efficient frequency sampling for this problem requires a non-evenly spaced (in frequency) set of snapshots, clustered near the low frequency region. Indeed (this will be shown subsequently) the electromagnetic problem varies more rapidly in 
Table 1: User-defined PGD parameters for the electromagnetic test magnet problem simulation.

\begin{tabular}{cccc|cc|ccc}
\hline \multicolumn{3}{c|}{ PGD parameters } & \multicolumn{3}{|c|}{ Parametric domains } & \multicolumn{3}{|c}{ Spatial domain } \\
\hline$I_{N}^{A}$ & tol & $I_{F P}$ & $t o l_{F P}$ & $h_{\omega}$ & $h_{\gamma}$ & $h_{\Omega_{p}}$ & $p$ & $\xi$ \\
40 & $10^{-4}$ & 10 & $10^{-2}$ & $2 \cdot 10^{-5}$ & $6 \cdot 10^{-3}$ & $5 \cdot 10^{-3}$ & 4 & $10^{-3}$ \\
\hline
\end{tabular}

the low frequency range and, thus, this a priori knowledge of the problem at hand can be used to optimise the number and location of snapshots needed. It is important to stress that, differently from the PGD method, the EM-POD technique considers the parametric space $(\omega, \gamma)$ as a single dimension, avoiding the use of the HOSVD. In addition, the a priori knowledge of the solution, facilitates the selection of an efficient set of snapshots (i.e. use of a non-evenly spaced frequency) in order to reduce the computational cost of the offline stage. The TSVD is applied on the set of stored snapshots and truncated after 20 modes $(M=20)$. In addition, the mesh size $h_{\Omega_{p}}$, the polynomial order $p$ and the damping ratio $\xi$ are also required. This information regarding the EM-POD set-up is summarised in Table 2.

Table 2: User-defined EM-POD parameters for the electromagnetic test magnet problem simulation.

\begin{tabular}{cccc|ccc}
\hline EM-POD parameters & & \multicolumn{4}{|c}{ Snapshots } \\
\hline$f^{\text {snap }}[\mathrm{Hz}]$ & $\alpha_{\gamma}^{\text {snap }}$ & $N_{s}$ & $M$ & $h_{\Omega_{p}}$ & $p$ & $\xi$ \\
{$[10: 80: 1000,1400: 400: 5000]$} & {$[0.5,1.25,2]$} & 69 & 20 & $5 \cdot 10^{-3}$ & 4 & $10^{-3}$ \\
\hline
\end{tabular}

PGD and EM-POD, are then run and compared against the full order (reference) solution. Figure $7 \mathrm{a}$ shows three different cases, each one consisting of a frequency sweep, for three different values of $\alpha_{\gamma}$ used for scaling the electric conductivity. The agreement between the different techniques is clearly observed and, hence, the implementation of PGD and EM-POD is validated. Moreover, the error introduced by either of the ROM techniques is negligible and, crucially, controllable by the various tolerance values used in the analyses. As noted above, the scalar magnetic potential field is smooth throughout the entire frequency spectrum but with a sharp gradient observable in the low frequency range.
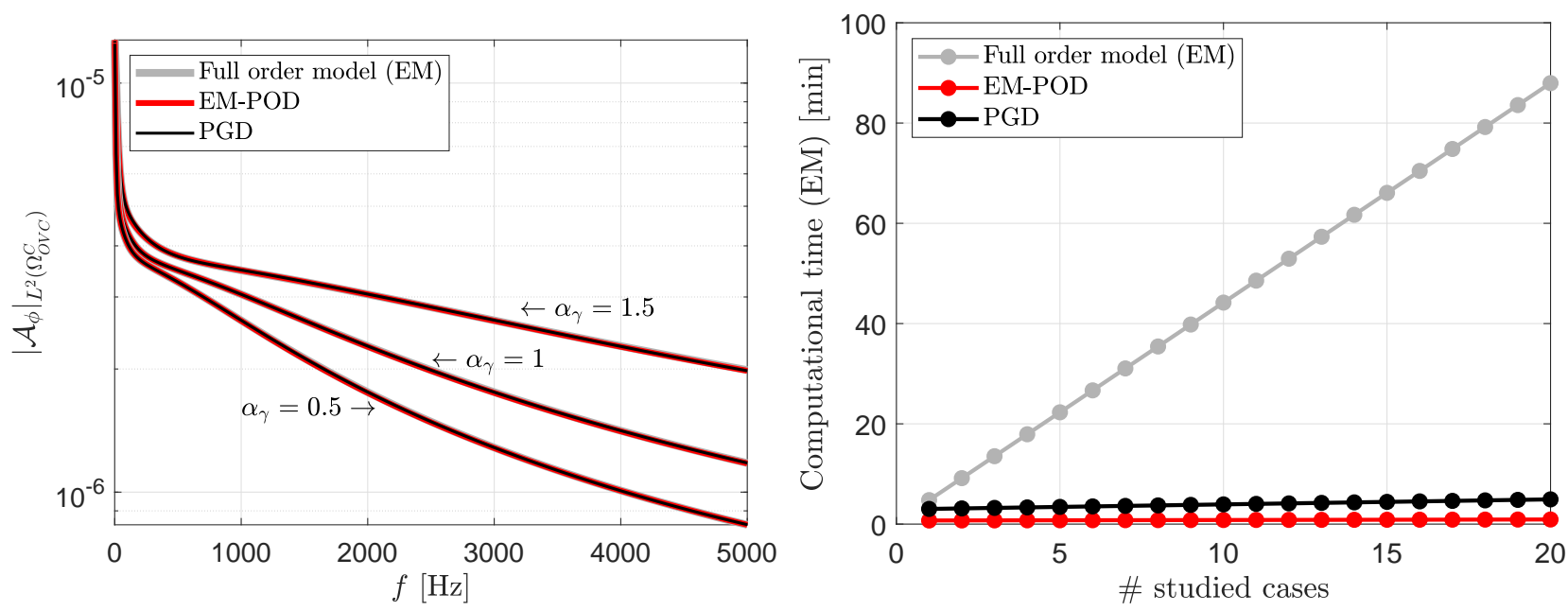

(a) Three different cases $\left(\alpha_{\gamma}=0.5, \alpha_{\gamma}=1\right.$ and $\left.\alpha_{\gamma}=1.5\right)$. (b) Total computational time required for each method to solve Plot of the L2 norm of the vector potential field in the OVC 20 studied cases (different parameter combinations). conducting shield $\left|\mathcal{A}_{\phi}\right|_{L^{2}\left(\Omega_{O V C}^{C}\right)}$.

Figure 7: Electromagnetic test magnet problem; comparison between PGD and EM-POD approximations against the reference full order model. 
In addition, Figure $7 \mathrm{~b}$ presents the computational time that the three methods (full order, EM-POD and PGD) require in order to complete the analysis of 20 different parametric cases. The full order model shows a clearly defined linear computational time cost, as each new case study implies the solution of an identical problem yet with a different parametric combination. However, the trend of the two ROM techniques is almost constant in cost due to the fact that the online costs of both methods are almost negligible compared to the offline costs. Note that, for this particular case, the time required for both ROM methods is comparable, being the PGD slightly more expensive (with the extra advantage that an explicit parametric solution field is obtained). Moreover, it has to be stressed that the number of snapshots used in the EM-POD method has been drastically minimised using the a priori knowledge of the solution, whereas the PGD technique does not require at all any previous knowledge of the problem. Furthermore, an increase in the number of parameters will slightly affect the PGD method whilst it could potentially seriously increase the offline (i.e. use of a HOSVD and a larger number of snapshots) and online (i.e. solution of a larger reduced system of equations) cost of the EM-POD technique.

For the PGD method, the offline calculations consist in obtaining and assimilating all terms appearing in the separable representation (22) and the online stage consists of a simple interpolation of the previously computed high-order parametric solution, that can be straightforwardly carried out in real time. As for the EM-POD method, the offline stage requires the computation of the snapshots, the TSVD and the non-parameter dependent assembly process (if the problem allows for an affine decomposition [36]). In the online stage, the parameter dependent assembly process is carried out in order to solve the reduced system of equations ( $\operatorname{size} M \times M$ ) that yields the electromagnetic response of the problem. Note that in this particular problem, the affine decomposition is possible, see (6), and thus, the online cost is still relatively small compared to the offline one. Note that this study has been performed with a smaller number of cases and then it has been extrapolated to 20 cases.

The ROM techniques can be queried in real time (especially efficient is PGD in this regard as only an interpolation process is carried out) to obtain the magnetic potential distribution that can be used to compute physically meaningful field quantities such as the transient magnetic field $\boldsymbol{B}_{0}^{A C}$ acting on the test magnet geometry, see Figure 8. It is interesting to see how the transient magnetic field is generated in the AC coils (red) and travels through the free space permeating across the conducting shields.

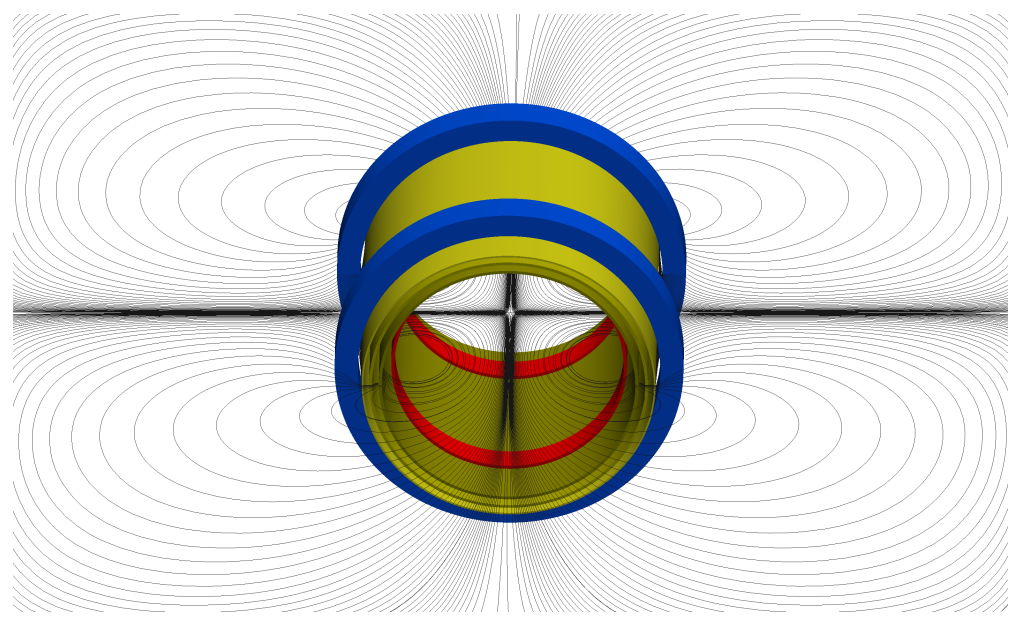

Figure 8: Electromagnetic test magnet problem; visualisation of the magnetic field $\left|\mathcal{B}_{0}^{A C}\right|$ contour lines on the axisymmetric plane. 3D representation of three MRI components; OVC, $77 \mathrm{~K}$ and $4 \mathrm{~K}$ radiation shields (gold tones), main DC coils (blue) and gradient AC coils (red). 


\subsubsection{Coupled magneto-mechanical problem}

After comparing both ROM techniques with the full order solution (for the electromagnetic problem), the more challenging fully coupled magneto-mechanical problem is now considered, where singularities in the solution field arise due to resonance. Regarding EM-POD, see flow chart in Figure 6, it combines the use of POD for the electromagnetic equations (with excellent accuracy with respect to the full order model as shown in Figure 7a) and a full order model for the mechanical equations. As a result, the EM-POD method does not introduce additional errors with respect to the full order model when solving the mechanical problem, although the mechanical solutions will not be exactly the same due to the fact that the source term for the mechanical problem is based on an approximated electromagnetic solution $\mathcal{A}_{\phi}^{P O D}$. However, important savings with respect to a complete full order solve are gained as the size of the electromagnetic problem (solved using POD) is generally orders of magnitude larger than that of the mechanical one (which is solved using the full order model).

Regarding PGD, the same strategy could have been used. The fully coupled magnetomechanical problem is solved and the user-defined parameters are presented in Table 3. Note that due to the sequential nature of the solution process, some of these PGD input parameters use the superscript $\left(\cdot{ }^{A}\right)$ or $\left(\cdot{ }^{u}\right)$ to denote whether they refer to the electromagnetic or mechanical problems, respectively. A new user-defined parameter, tol split, is needed in the mechanical problem in order to define the maximum size of the frequency subintervals in the resonance area. This parameter is used in the automatic adaptive frequency splitting, presented in [35], which consists in automatically detecting the numerical singularities associated with resonance and refining the frequency domain accordingly, without the need of any a priori knowledge of the solution. Note that this splitting process is different to that presented by the authors in [35]. Previously, the PGD algorithm was very sensitive to the frequency subinterval, where each subinterval had to contain a few natural frequencies as centred as possible. However, with the staggered PGD presented in this paper, the algorithm becomes more robust and it is possible to apply a simpler splitting procedure based on the location of the smallest and largest eigenvalues of the mechanical problem contained within the frequency range of interest.

As displayed in Figure 9, a complete eigenvalue analysis of the problem is too generic and does not actually provide reliable information about the truly excited resonant frequencies. Instead, frequencies with high multiplicity and non-excited resonance frequencies may be obtained. For the implementation of the PGD algorithm, the first and last eigenvalues inside the frequency range of interest are computed, which will define the resonance region of our problem. Once this region is located, uniform splitting is performed using tolsplit. The above eigenvalue analysis is not a computational burden as a result of (i) the consideration of each individual conducting shield one at a time and (ii) the need to extract only the maximum and minimum values within the frequency range of interest.

Table 3: User-defined parameters for the test magnet problem simulation.

\begin{tabular}{ccccc|ccccc|ccc}
\hline \multicolumn{4}{c|}{ PGD parameters } & \multicolumn{4}{c|}{ Parametric domains } & \multicolumn{3}{c}{ Spatial domain } \\
\hline$I_{N}^{A}$ & $I_{N}^{u}$ & tol $_{N}^{A}$ & tol $l_{N}^{u}$ & $I_{F P}$ & tol $_{F P}$ & $h_{\omega}$ & tol $_{\omega}^{\text {split }}$ & $h_{\gamma}$ & $h_{B_{0}}$ & $h_{\Omega_{p}}$ & $p$ & $\xi$ \\
40 & 60 & $10^{-4}$ & $10^{-5}$ & 10 & $10^{-2}$ & $2 \cdot 10^{-5}$ & $20 \%$ & $6 \cdot 10^{-3}$ & $8 \cdot 10^{-3}$ & $5 \cdot 10^{-3}$ & 4 & $10^{-3}$ \\
\hline
\end{tabular}

The staggered PGD algorithm can then be applied to the test magnet problem in order to obtain (offline) the magnetic potential and the displacement field for the two physics considered. In the context of an MRI problem, two important (spatially integrated) quantities of interest are typically reported, namely, the dissipated or Ohmic power $P_{\Omega^{C}}^{0}$ and the kinetic energy $E_{\Omega^{C}}^{k}$, 

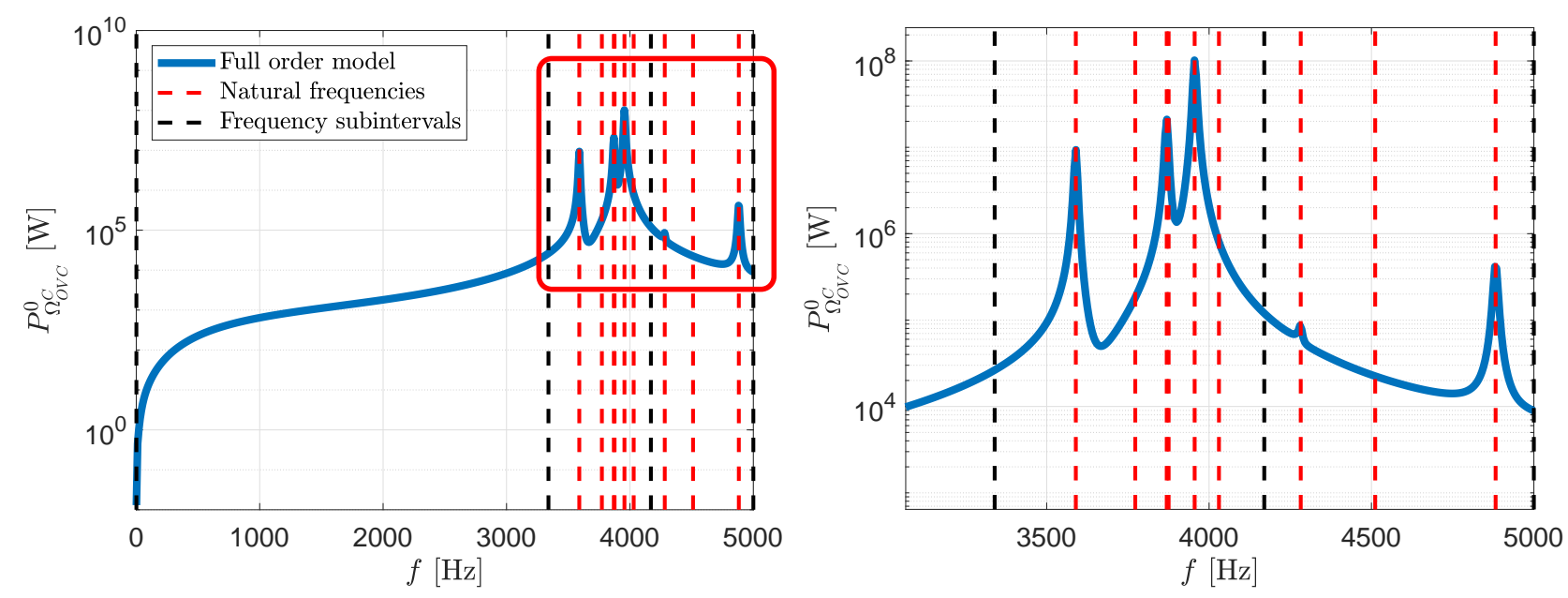

Figure 9: Test magnet problem; natural frequencies (red) obtained from eigenvalue analysis and frequency subintervals (black) defined through frequency splitting. Right figure shows a zoomed view in the resonance region (red rectangle). Results shown are for a mesh of $2.9 \mathrm{~K}$ triangular elements using a polynomial order $p=4$.

which can be both computed as a postprocess of the solution fields as

$$
P_{\Omega^{C}}^{0}:=\frac{1}{2} \int_{\Omega^{C}} \gamma\left|\mathcal{E}^{A C}\right|^{2} \mathrm{~d} \Omega, \quad E_{\Omega^{C}}^{k}:=\frac{1}{2} \int_{\Omega^{C}} \rho \omega^{2}\left|\boldsymbol{u}^{A C}\right|^{2} \mathrm{~d} \Omega .
$$

The results for these two quantities are presented in Figure 10, where $P_{\Omega^{C}}^{0}$ (left) and $E_{\Omega^{C}}^{k}$ (right) are shown for each of the three conducting shields. A series of curves are displayed in each figure, where the black solid lines on the background denote the full order model solution ${ }^{11}$ for different sets of parameters $B_{0}$ and $\alpha_{\gamma}$. The coloured lines lying on top refer to the different PGD solutions for the equivalent sets of parameters used in the full order model. Note that the specific sets of parameters used are presented in the figure legend. These results show that the PGD approximation is able to accurately reproduce the behaviour of the full order model, splitting the frequency range only where it is required and accurately capturing all the singularities of the response. These results clear demonstrate the validity of the reported staggered PGD model.

Figure 11 shows the comparison in terms of the total computational time required by the staggered PGD model and the full order model. First, the total time is shown in Figure 11a, where a clear time reduction is observed between the full order model and the PGD approach even if just a few cases (parameter combinations) are required. Moreover, the online PGD cost is no longer negligible as it was when only considering the electromagnetic problem, see Figure $7 \mathrm{~b}$. The reason is that the online cost now includes the computation of the integrated quantities (38). Figure 11b shows the time reduction in percentage obtained for the PGD technique using the full order method as a reference, where a great reduction of approximately $85 \%$ is obtained when studying, for instance, 20 different cases. PGD is based on the general idea of maximising the computational effort during the offline stage in order to reduce as much as possible online computing tasks. In particular, the online stage reduces to a mere interpolation process that can be easily conducted in real time and displayed via user-friendly application tool (the reader is referred to [35] for an example of an online PGD application tool).

\subsubsection{Staggered versus monolithic PGD technique}

Now that the staggered PGD algorithm has been validated against the full order model, this section will focus on the advantages of exploiting the staggered nature of the magneto-

\footnotetext{
${ }^{11}$ Identical results are obtained when using the EM-POD combined with a mechanical full order solution.
} 

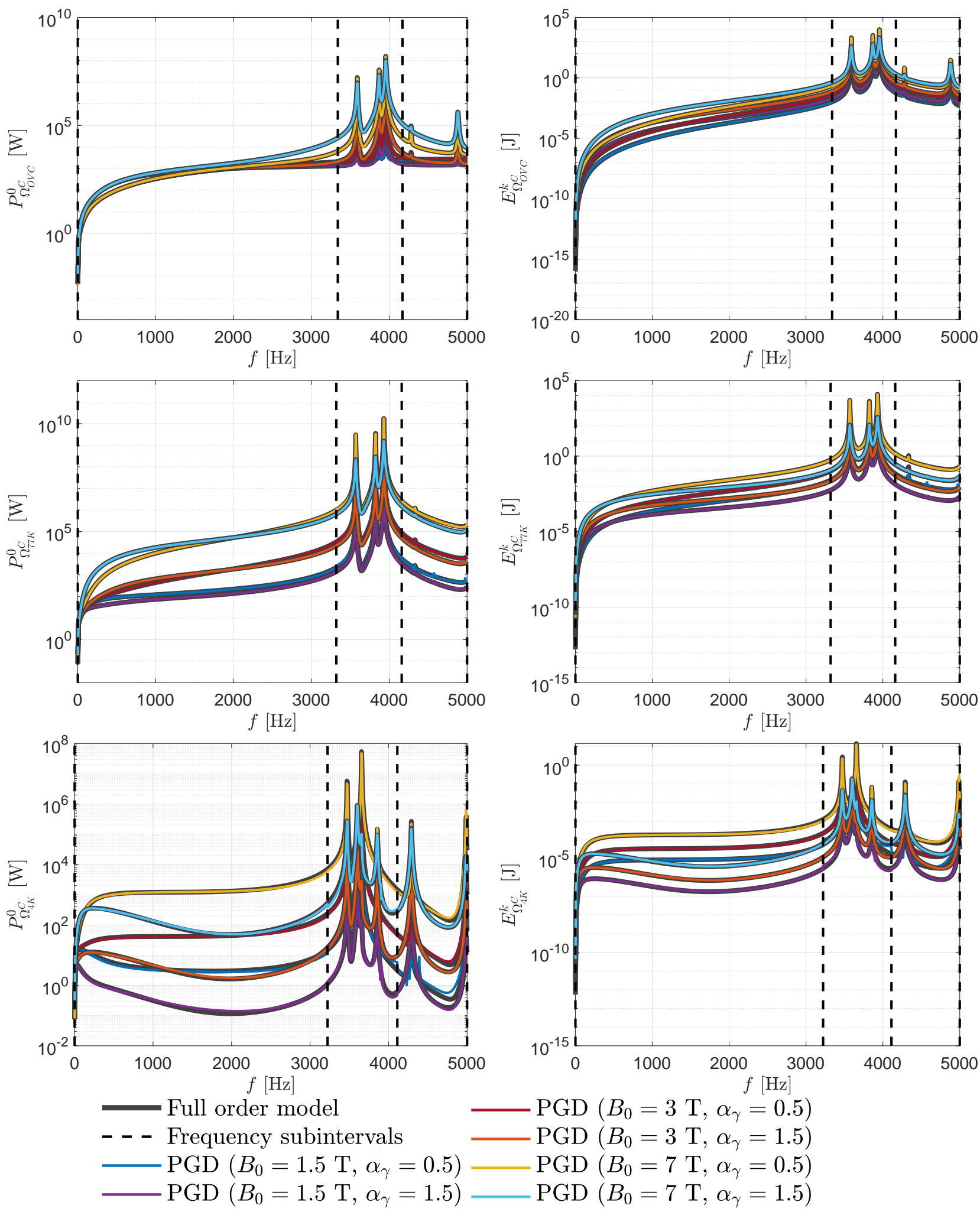

$\operatorname{PGD}\left(B_{0}=3 \mathrm{~T}, \alpha_{\gamma}=0.5\right)$

$\operatorname{PGD}\left(B_{0}=3 \mathrm{~T}, \alpha_{\gamma}=1.5\right)$

$\operatorname{PGD}\left(B_{0}=7 \mathrm{~T}, \alpha_{\gamma}=0.5\right)$

$\operatorname{PGD}\left(B_{0}=7 \mathrm{~T}, \alpha_{\gamma}=1.5\right)$

Figure 10: Test magnet problem; comparison between PGD solution and full order model. Plot of the dissipated power $P_{\Omega^{C}}^{0}$ and kinetic energy $E_{\Omega^{C}}^{k}$ in the three conducting shields OVC, $77 \mathrm{~K}$ and $4 \mathrm{~K}$ for six different cases.

mechanical problem. This new staggered PGD approach will be contrasted against the monolithic PGD technique presented in [35], where the PGD algorithm was applied simultaneously to both physics in a monolithic manner.

The convergence of the PGD methodology is often measured by the contribution of the last computed mode [19]. This means that if the last computed mode has a weight that is small enough, this particular mode will not be as important as compared to the previously 


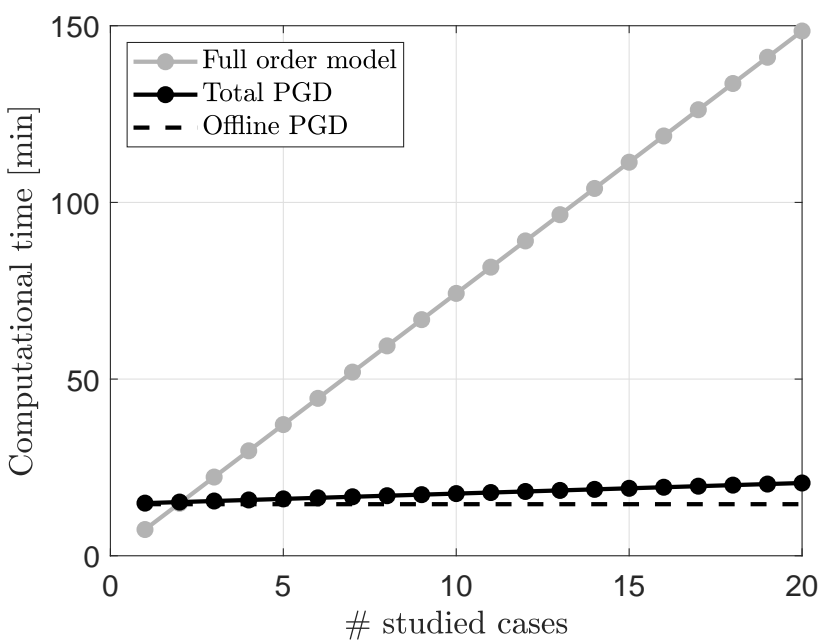

(a) Total time (solid line) and offline time (dashed line) for the three methods.

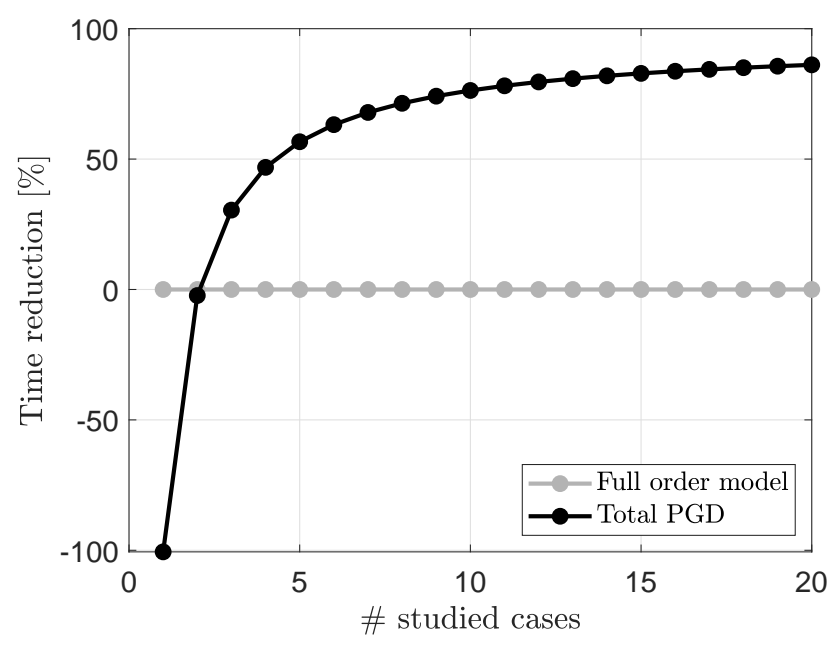

(b) Time reduction (\%) obtained for each method.

Figure 11: Test magnet problem; study of the computational time taken by the full order model and both offline and online stages of the PGD technique. 700 frequencies have been sampled for each case of study using the full order model.

accumulated and, therefore, the greedy algorithm can be safely stopped. The error norms used to quantify this modal contribution are defined as

$$
e_{n}^{A}=\frac{\beta_{\mathfrak{A}_{\phi}}^{n}}{\sum_{m=1}^{n} \beta_{\mathcal{A}_{\phi}}^{[m]}}, \quad e_{n}^{u}=\frac{\beta_{\boldsymbol{u}}^{n}}{\sum_{m=1}^{n} \beta_{\boldsymbol{u}}^{[m]}},
$$

where the index $n$ (i.e. $n=1, \ldots, N_{A}$ for electromagnetics and $n=1, \ldots, N_{u}$ for mechanics) denotes a particular PGD mode. These error norms give an overall idea of the efficiency of the PGD algorithm and, thus, they are useful in order to compare the previously published monolithic approach [35] against the new staggered PGD approach, see Figure 12.

For the electromagnetic equations (Figure 12 left), two convergence curves are presented, monolithic PGD $e_{\text {mono }}^{A}$ and staggered PGD $e_{\text {stag }}^{A}$. It is clear that the staggered PGD approach converges considerably faster and to a lower modal contribution. As for the mechanics equations (Figure 12 right), one convergence curve is displayed for the monolithic PGD $e_{\text {mono }}^{u}$ model and three additional curves for the staggered approach $e_{\text {stag }}^{u}$, namely, one per shield $\left(e_{\text {stag }}^{u, O V C}, e_{\text {stag }}^{u, 77 K}\right.$ and $\left.e_{\text {stag }}^{u, 4 K}\right)$. Note that in this case the total number of modes is actually the same for both approaches, 60 modes for the monolithic PGD approach and $20 \cdot 3=60$ for the staggered PGD approach. However, recall that for the staggered PGD approach, the time required for the computation of each mode is lower as a result of considering every shield individually (in parallel). Moreover, the error norms converge to a lower modal contribution. All in all, from these results it can be concluded that the staggered PGD methodology offers a very competitive alternative in terms of accuracy, robustness and computational time.

An additional benefit of the staggered PGD approach that must be emphasised is the flexibility of the PGD algorithm, enabling to set different control parameters for electromagnetics and mechanics and allowing the automatic split of the frequency domain only for the mechanics. This flexibility permits the use of different convergence criteria for the PGD, concentrating the computational effort where it is truly needed.

\subsection{Full magnet problem}

The full magnet problem represents a more realistic MRI scanner configuration of great interest to industry. Again, the geometry, material properties and boundary conditions were 

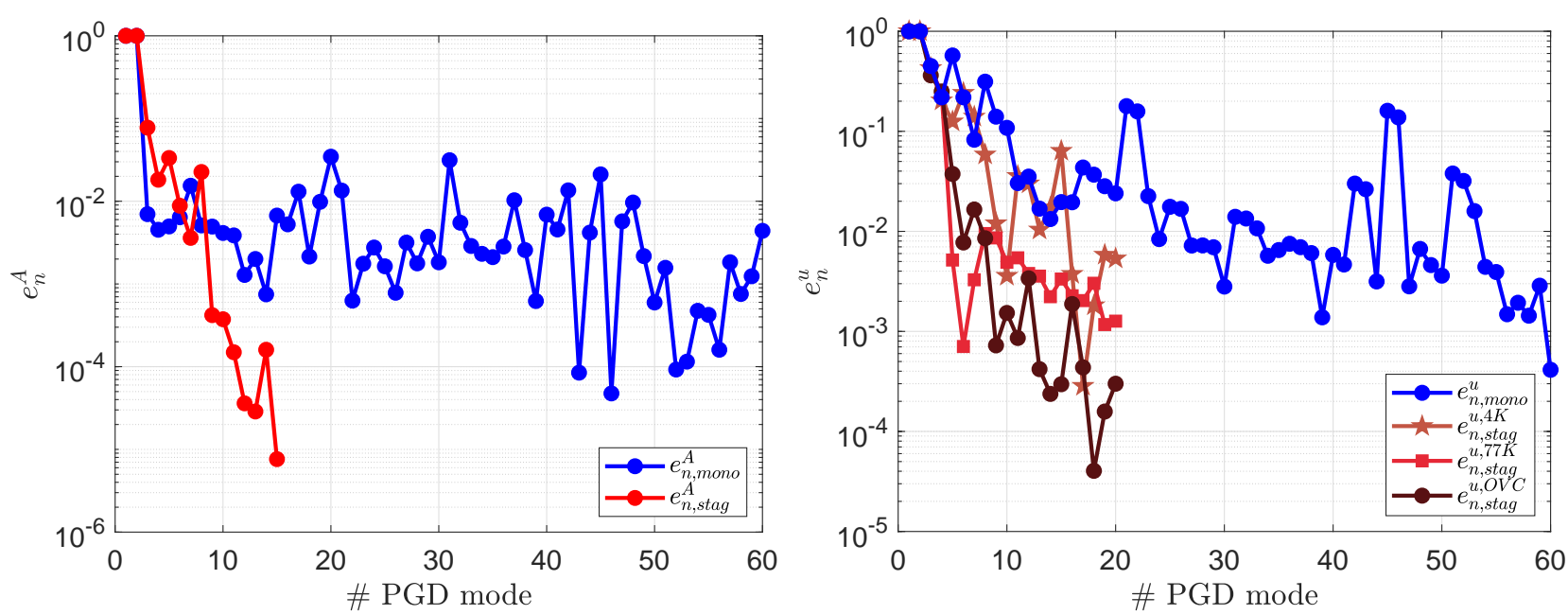

Figure 12: Test magnet problem; Last mode contribution for electromagnetics $e^{A}$ and mechanics $e^{u}$. Comparison between monolithic PGD $e_{\text {mono }}$ and staggered PGD $e_{\text {stag }}$ approaches. Both PGD algorithms only depending on space $(r, z)$ and frequency $\omega$ with no splitting in the frequency domain.

already presented in [35], but they are also described here for completeness. This problem is also represented by a set of main and gradient coils and the three conducting shields OVC, $77 \mathrm{~K}$ and $4 \mathrm{~K}$ which are now more realistically represented by closed cylindrical shells. Each shield has different material and geometrical parameters that cannot be specified due to confidentiality issues. However, the approximate values are $\mu_{*} \approx 10^{-7} \mathrm{H} / \mathrm{m}, \gamma_{*} \approx 10^{6} \mathrm{~S} / \mathrm{m}, \rho \approx 10^{3} \mathrm{Kg} / \mathrm{m}^{3}$, $E \approx 10^{9} \mathrm{~Pa}$ and $\nu \approx 0.3$. Similarly than in Section 5.1, the axisymmetric formulation [13] follows from assuming rotational symmetry of the weak form expressed in 3D cylindrical coordinates and, as such, $r=0$ does not form a boundary of the computational domain $\Omega_{p}$.

The user-defined parameters in order to compute the PGD offline solution for the full magnet geometry are presented in Table 4. The parameters related to the parametric and spatial domains are almost identical to the ones employed for the test magnet problem (see Table 3). However, there are substantial changes in the PGD parameters used, mainly in the computation of the offline electromagnetic problem. As expected, the increased complexity of this problem in comparison to the test magnet problem leads to a greater number of PGD modes in order to obtain an accurate offline solution. As for the offline mechanical problem, the total maximum number of modes per frequency subinterval and per shield $I_{N}^{u}$ has also been increased with respect to the test magnet problem.

Table 4: User-defined PGD parameters for the full magnet problem simulation.

\begin{tabular}{cccccc|cccc|ccc}
\hline \multicolumn{4}{c|}{ PGD parameters } & \multicolumn{4}{c|}{ Parametric domains } & \multicolumn{3}{c}{ Spatial domain } \\
\hline$I_{N}^{A}$ & $I_{N}^{u}$ & tol $_{N}^{A}$ & tol $l_{N}^{u}$ & $I_{F P}$ & tol $_{F P}$ & $h_{\omega}$ & tol $_{\omega}^{\text {split }}$ & $h_{\gamma}$ & $h_{B_{0}}$ & $h_{\Omega_{p}}$ & $p$ & $\xi$ \\
60 & 40 & $10^{-6}$ & $10^{-5}$ & 10 & $10^{-2}$ & $2 \cdot 10^{-5}$ & $20 \%$ & $6 \cdot 10^{-3}$ & $8 \cdot 10^{-3}$ & $7 \cdot 10^{-3}$ & 4 & $10^{-3}$ \\
\hline
\end{tabular}

A similar comparison (full order versus EM-POD and PGD) to that presented in Figure 7 was repeated for this more challenging geometry, but as the conclusions were the same they have no been repeated. Instead, the post-processed quantities of interest, dissipated power $P_{\Omega^{C}}^{0}$ and kinetic energy $E_{\Omega^{C}}^{k}(38)$, are presented in Figure 13 for the entire coupled magneto-mechanical problem. Similarly to the test magnet problem, the full order model is denoted with a black solid line for all the different cases, whilst the PGD solution is plotted with coloured lines that can be associated with different combinations of parameters $\left(B_{0}, \gamma\right)$ in the legend. From Figure 13, it can be concluded that the PGD approximation is able to accurately replicate the full order solution and locate all the resonance singularities for this complex simulation. 

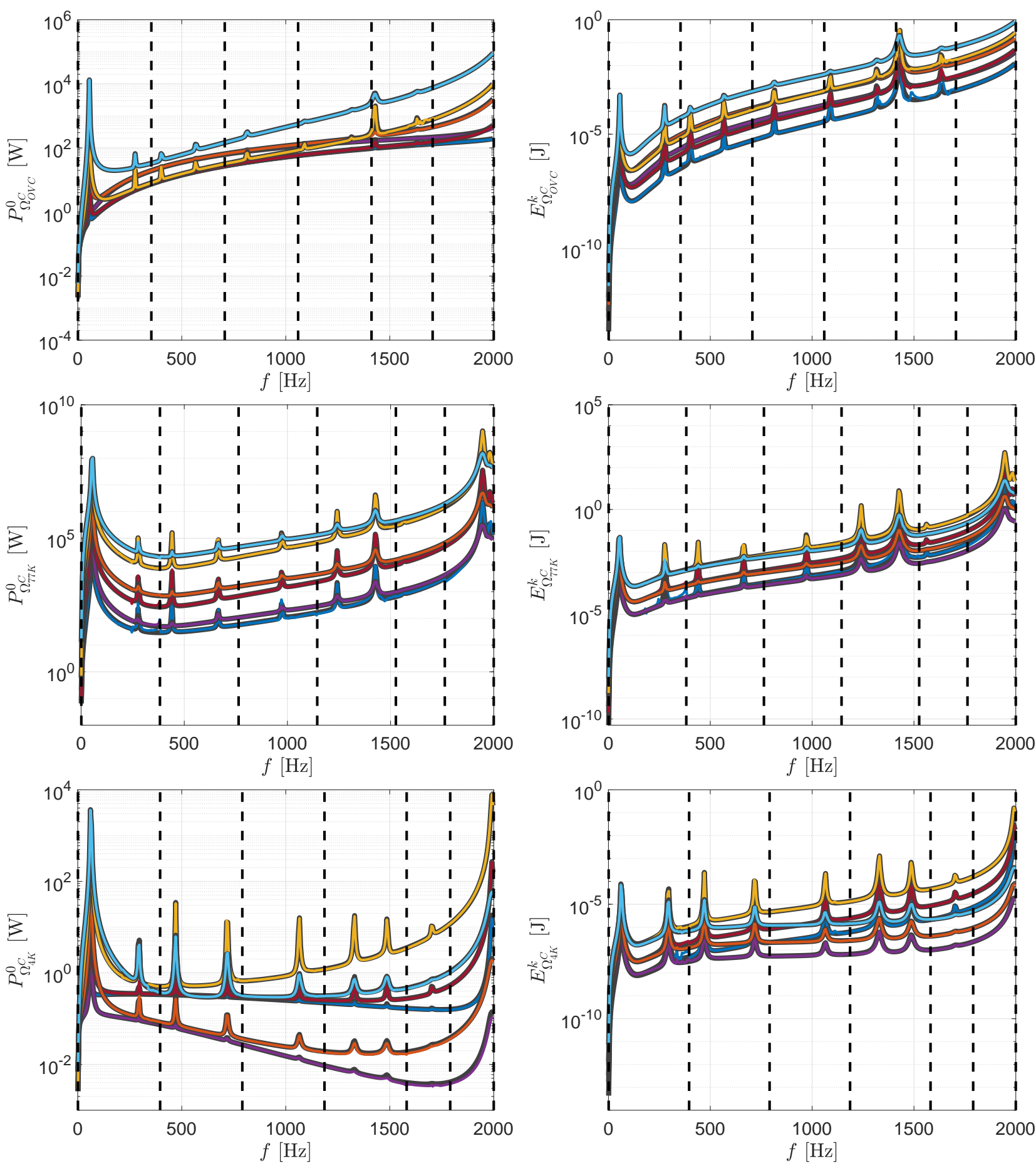

Full order model

$\operatorname{PGD}\left(B_{0}=3 \mathrm{~T}, \alpha_{\gamma}=0.5\right)$

- - Frequency subintervals

PGD $\left(B_{0}=1.5 \mathrm{~T}, \alpha_{\gamma}=0.5\right)$

PGD $\left(B_{0}=3 \mathrm{~T}, \alpha_{\gamma}=1.5\right)$

PGD $\left(B_{0}=7 \mathrm{~T}, \alpha_{\gamma}=0.5\right)$

PGD $\left(B_{0}=1.5 \mathrm{~T}, \alpha_{\gamma}=1.5\right)$

PGD $\left(B_{0}=7 \mathrm{~T}, \alpha_{\gamma}=1.5\right)$

Figure 13: Full magnet problem; comparison between PGD solution and full order model. Plot of the dissipated power $P_{\Omega^{C}}^{0}$ and kinetic energy $E_{\Omega^{C}}^{k}$ in the three conducting shields OVC, $77 \mathrm{~K}$ and $4 \mathrm{~K}$ for six different cases.

The computational time required to compute the different cases appearing in Figure 13 has been used to generate Figure 14, where the full order model and the PGD method are compared considering up to 20 cases (different parameter combinations). These results show a more costly PGD method with respect to the full order model than for the test magnet geometry, see Figure 11. However, it is clearly seen how with just 6 cases the PGD approach is already cheaper than the full order model. Moreover, massive savings can be achieved if more 
cases need to be studied, reaching approximately a $70 \%$ reduction.

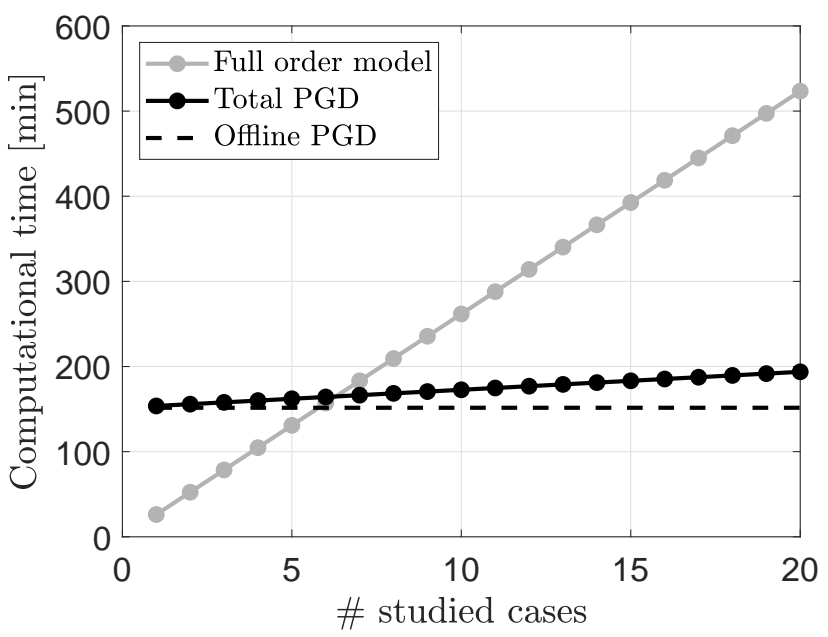

(a) Total time (solid line) and offline time (dashed line) for the full order model and staggered PGD approaches.

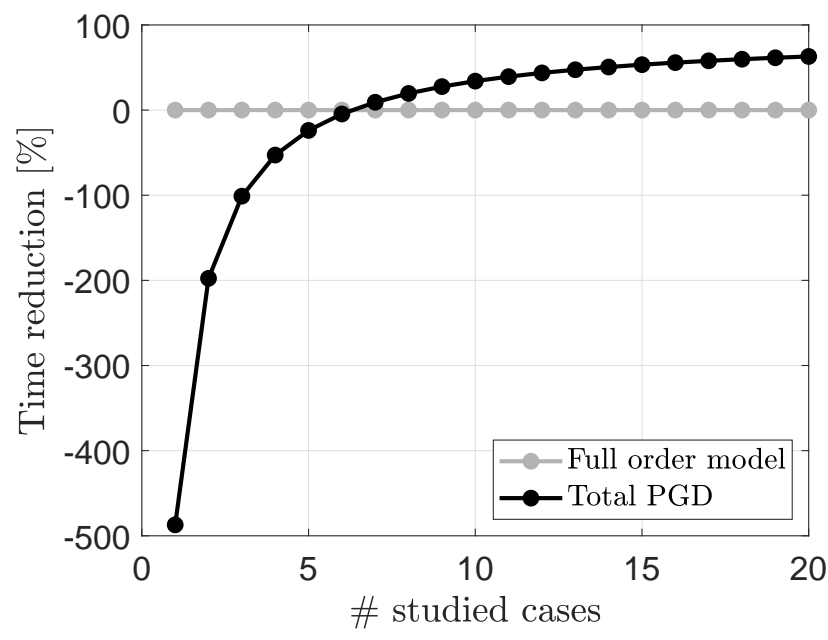

(b) Time reduction (\%) obtained with the PGD method.

Figure 14: Full magnet problem; study of the computational time taken by the full order model and the staggered PGD approach. 400 frequencies have been sampled for each case of study.

Although integrated quantities, such as dissipated power $P_{\Omega^{C}}^{0}$ and kinetic energy $E_{\Omega^{C}}^{k}(38)$, are important quantities of interest for industry, they are not the only quantities required for design. Other field quantities such as magnetic field displacements, stresses and eddy current distributions are also taken into account as part of the design process. It is here where an additional advantage of the PGD methodology can be exploited, by pre-computing explicit separable expressions of the solution fields which can be then queried in real time. Figure 15 shows the eddy current distribution $\boldsymbol{J}^{0}$ inside the conductors, computed as $\boldsymbol{J}^{0}=\gamma \boldsymbol{E}^{A C}$, for $B_{0}=1.5 \mathrm{~T}, \gamma=1$ and different values of the frequency of excitation $f$. A typical phenomenon can be observed in this Figure; the skin depth effect. For low frequencies $(100 \mathrm{~Hz})$ see how the eddy current field is constant across the shield's thickness. However, in the high-frequency region (2000 and $4000 \mathrm{~Hz}$ ) the skin depth effect becomes more dominant, modifying the eddy current distribution within the radiation shields by concentrating the eddy currents in the interfaces of the shields. The fact that sharp changes are observed in a small part of the shields' thickness makes the problem more demanding from the computational point of view, requiring the careful combination of high fidelity space-time discretisations and reduced order computational models.

Finally, it is interesting to demonstrate a further capability of the PGD methodology, related to its ability to efficiently conduct online multiple-queries (in real time) to the high-dimensional parametric offline solution. As an example, it is possible to compute and plot output response surfaces (for a given quantity of interest) when arbitrarily varying the set of input parameters used in the construction of the PGD offline solution. Figure 16 shows three different response surfaces of the post-processed output power in the OVC shield $P_{\Omega_{O V C}^{C}}^{0}$ for different combinations of frequency $f[\mathrm{~Hz}]$, strength of static magnetic field $B_{0}[\mathrm{~T}]$ and dimensionless scaling of electric conductivity $\alpha_{\gamma}$. Note that although it is technically possible to compute these response surfaces with the full order model, the computational time required would be extremely high since a large number of sample points (2000 in this case) can be typically needed in order to display the surface with an acceptable level of resolution.

\section{Conclusion}

This paper has presented a new Reduced Order Modelling (ROM) Proper Generalised Decomposition (PGD) method for use in the design phase of new MRI scanner configurations. 


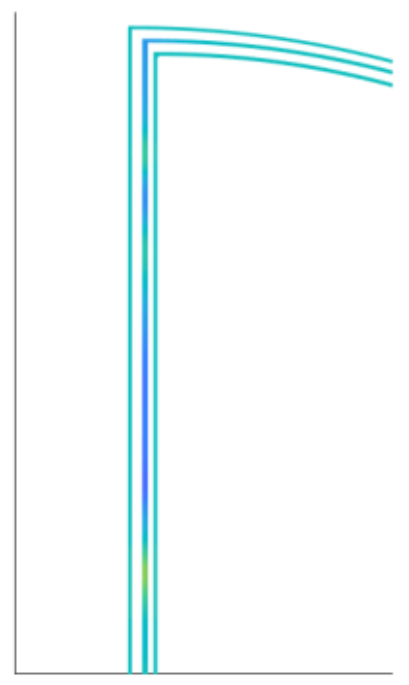

(a) $f=100 \mathrm{~Hz}$.

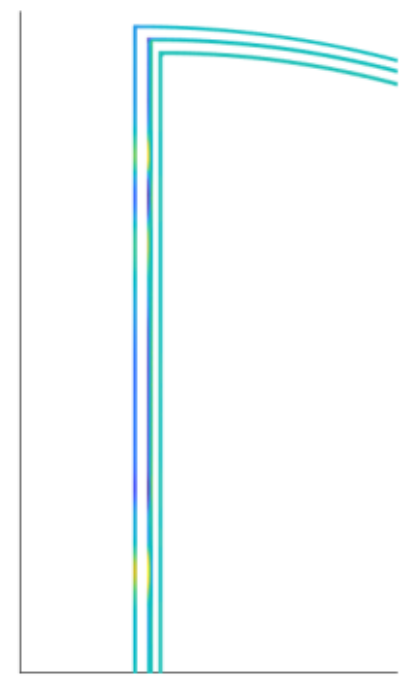

(b) $f=2000 \mathrm{~Hz}$.

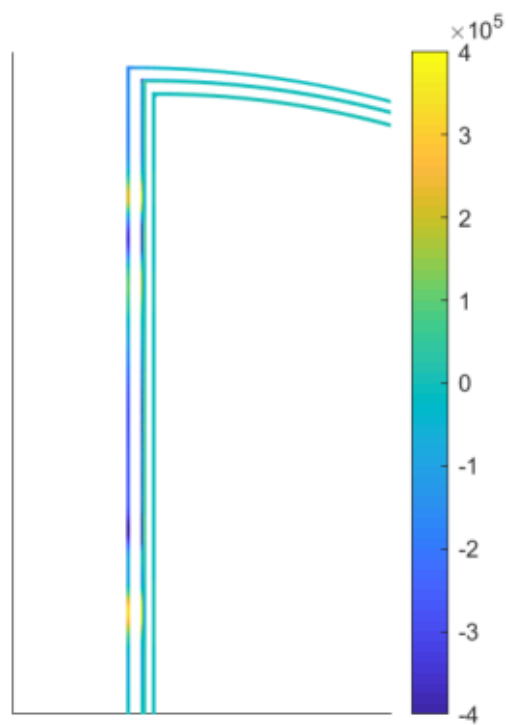

(c) $f=4000 \mathrm{~Hz}$.

Figure 15: Full magnet problem; Eddy current distribution within the radiation (conducting) shields for different frequencies $f, B_{0}=1.5 \mathrm{~T}$ and $\gamma=1$.

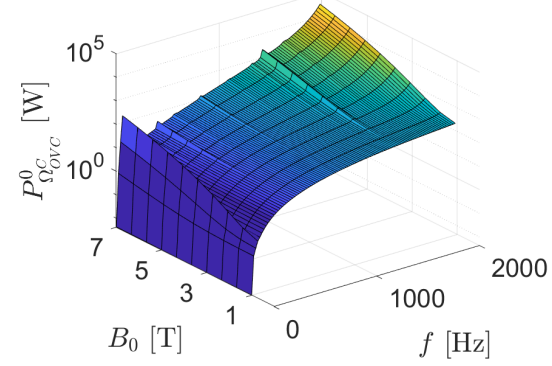

(a) $f / B_{0}$ plot for $\alpha_{\gamma}=1.5$.

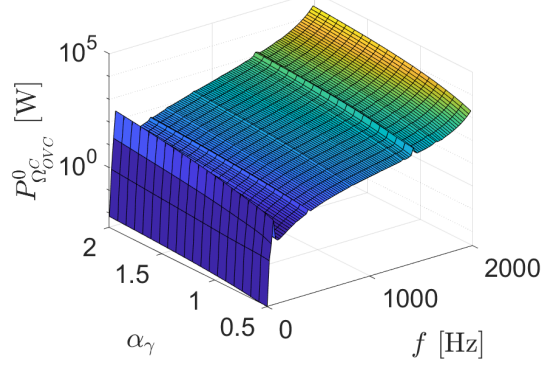

(b) $f / \alpha_{\gamma}$ plot for $B_{0}=5 \mathrm{~T}$.

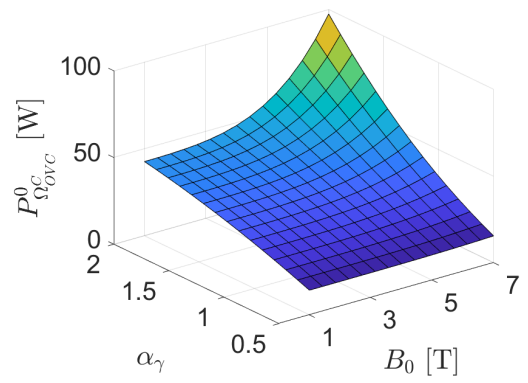

(c) $B_{0} / \alpha_{\gamma}$ plot for $f=500 \mathrm{~Hz}$.

Figure 16: Full magnet problem; response surfaces of the output power in the OVC shield $P_{\Omega_{O V C}^{C}}^{0}$ for different values of $f, B_{0}$ and $\alpha_{\gamma}$.

The methodology builds upon previous work developed by the authors in [35] where a PGDfrequency based methodology was developed and it is here enhanced by considering two further material parameters as part of the high-dimensional parametric PGD offline solution, namely, the electrical conductivity and the strength of the static magnetic field. The new (reduced order) PGD methodology has been validated against a reference (full order) solution in terms of accuracy and computational time, where it has been clearly shown how the PGD method can drastically optimise the multiple-query online stage without sacrificing accuracy. Moreover, the staggered nature of the underlying magneto-mechanical problem has been exploited in this work, in order to obtain a sequential PGD algorithm that has been proven to be both efficient and robust.

The a priori PGD algorithm has also been compared in terms of the electromagnetic output against an alternative a posteriori ROM method specifically designed for this problem, the electromagnetic Proper Orthogonal Decomposition (EM-POD) method. This study concludes that a massive time reduction can be obtained in the electromagnetic problem with both ROM approaches. When considering the entire coupled magneto-mechanical problem, the EM-POD strategy developed in [36] is still able to greatly reduce the computational cost of the problem at the expense of increasing the calculations done in the online POD stage. On the other hand, the staggered PGD presented in this paper is able to simulate the entire coupled magnetomechanical problem, obtaining an explicit expression of the solution fields, conserving the real 
time attractiveness during the online PGD stage and allowing to output sensitivity maps. The next step of our work will focus on extending the PGD formulation to include geometrical changes, for instance the thickness of the conducting shields, as extra parameters of the PGD offline solution, in the search of a computational metamodel which allows for real time simulations, thus minimising human intervention.

\section{Acknowledgements}

The authors would like to thank the European Commission for the funding received through the Marie Sklodowska-Curie Innovative Training Network AdMoRe with grant number 675919.

\section{Appendix A. Formulation and implementation of the 5D PGD}

This appendix contains the detailed PGD formulation for the two physics presented in this paper, namely, electromagnetics and mechanics. The continuous expressions of the residual forms for each of the physics of the problem are defined and expanded first. The FEM discrete expressions are then presented in the same way as they have been implemented in order to generate the results of numerical examples section.

\section{Appendix A.1. Electromagnetics}

The electromagnetic residuals defined in (26) can be expanded taking advantage of the separability of the terms, see (23). Hence, these residuals can be shown to be

$$
\begin{aligned}
& \mathcal{R}_{f_{\mathfrak{A}_{\phi}}}\left(\delta f_{\mathfrak{A}_{\phi}} ; \mathcal{A}_{\phi}^{n-1}, f_{\mathfrak{A}_{\phi}}, g_{\mathfrak{A}_{\phi}}, h_{\mathfrak{A}_{\phi}}\right)=W_{K}^{A}\left(f_{\mathfrak{A}_{\phi}}, \delta f_{\mathfrak{A}_{\phi}}\right) G_{0}^{A}\left(g_{\mathfrak{A}_{\phi}}, g_{\mathfrak{A}_{\phi}}\right) H_{0}^{A}\left(h_{\mathfrak{A}_{\phi}}, h_{\mathfrak{A}_{\phi}}\right) \\
& +\mathrm{i} W_{C}^{A}\left(f_{\mathfrak{A}_{\phi}}, \delta f_{\mathfrak{A}_{\phi}}\right) G_{1}^{A}\left(g_{\mathfrak{A}_{\phi}}, g_{\mathfrak{A}_{\phi}}\right) H_{1}^{A}\left(h_{\mathfrak{A}_{\phi}}, h_{\mathfrak{A}_{\phi}}\right) \\
& -S^{A}\left(\delta f_{\mathcal{A}_{\phi}}\right) G_{0}^{A}\left(1, g_{\mathfrak{A}_{\phi}}\right) H_{0}^{A}\left(1, h_{\mathcal{A}_{\phi}}\right) \\
& +\sum_{m=1}^{n-1}\left[W_{K}^{A}\left(F_{\mathfrak{A}_{\phi}}^{[m]}, \delta f_{\mathfrak{A}_{\phi}}\right) G_{0}^{A}\left(G_{\mathcal{A}_{\phi}}^{[m]}, g_{\mathfrak{A}_{\phi}}\right) H_{0}^{A}\left(H_{\mathfrak{A}_{\phi}}^{[m]}, h_{\mathfrak{A}_{\phi}}\right)\right. \\
& \left.+\mathrm{i} W_{C}^{A}\left(F_{\mathfrak{A}_{\phi}}^{[m]}, \delta f_{\mathfrak{A}_{\phi}}\right) G_{1}^{A}\left(G_{\mathfrak{A}_{\phi}}^{[m]}, g_{\mathfrak{A}_{\phi}}\right) H_{1}^{A}\left(H_{\mathfrak{A}_{\phi}}^{[m]}, h_{\mathfrak{A}_{\phi}}\right)\right], \\
& \mathcal{R}_{g_{\mathfrak{A}_{\phi}}}\left(\delta g_{\mathfrak{A}_{\phi}} ; \mathfrak{A}_{\phi}^{n-1}, f_{\mathfrak{A}_{\phi}}, g_{\mathfrak{A}_{\phi}}, h_{\mathfrak{A}_{\phi}}\right)=W_{K}^{A}\left(f_{\mathfrak{A}_{\phi}}, f_{\mathfrak{A}_{\phi}}\right) G_{0}^{A}\left(g_{\mathfrak{A}_{\phi}}, \delta g_{\mathfrak{A}_{\phi}}\right) H_{0}^{A}\left(h_{\mathfrak{A}_{\phi}}, h_{\mathfrak{A}_{\phi}}\right) \\
& +\mathrm{i} W_{C}^{A}\left(f_{\mathfrak{A}_{\phi}}, f_{\mathfrak{A}_{\phi}}\right) G_{1}^{A}\left(g_{\mathfrak{A}_{\phi}}, \delta g_{\mathfrak{A}_{\phi}}\right) H_{1}^{A}\left(h_{\mathcal{A}_{\phi}}, h_{\mathfrak{A}_{\phi}}\right) \\
& -S^{A}\left(f_{\mathcal{A}_{\phi}}\right) G_{0}^{A}\left(1, \delta g_{\mathfrak{A}_{\phi}}\right) H_{0}^{A}\left(1, h_{\mathfrak{A}_{\phi}}\right) \\
& +\sum_{m=1}^{n-1}\left[W_{K}^{A}\left(F_{\mathfrak{A}_{\phi}}^{[m]}, f_{\mathfrak{A}_{\phi}}\right) G_{0}^{A}\left(G_{\mathcal{A}_{\phi}}^{[m]}, \delta g_{\mathfrak{A}_{\phi}}\right) H_{0}^{A}\left(H_{\mathfrak{A}_{\phi}}^{[m]}, h_{\mathcal{A}_{\phi}}\right)\right. \\
& \left.+\mathrm{i} W_{C}^{A}\left(F_{\mathfrak{A}_{\phi}}^{[m]}, f_{\mathfrak{A}_{\phi}}\right) G_{1}^{A}\left(G_{\mathfrak{A}_{\phi}}^{[m]}, \delta g_{\mathfrak{A}_{\phi}}\right) H_{1}^{A}\left(H_{\mathfrak{A}_{\phi}}^{[m]}, h_{\mathfrak{A}_{\phi}}\right)\right], \\
& \mathcal{R}_{h_{\mathfrak{A}_{\phi}}}\left(\delta h_{\mathfrak{A}_{\phi}} ; \mathcal{A}_{\phi}^{n-1}, f_{\mathfrak{A}_{\phi}}, g_{\mathfrak{A}_{\phi}}, h_{\mathfrak{A}_{\phi}}\right)=W_{K}^{A}\left(f_{\mathfrak{A}_{\phi}}, f_{\mathfrak{A}_{\phi}}\right) G_{0}^{A}\left(g_{\mathfrak{A}_{\phi}}, g_{\mathfrak{A}_{\phi}}\right) H_{0}^{A}\left(h_{\mathfrak{A}_{\phi}}, \delta h_{\mathfrak{A}_{\phi}}\right) \\
& +\mathrm{i} W_{C}^{A}\left(f_{\mathfrak{A}_{\phi}}, f_{\mathfrak{A}_{\phi}}\right) G_{1}^{A}\left(g_{\mathfrak{A}_{\phi}}, g_{\mathfrak{A}_{\phi}}\right) H_{1}^{A}\left(h_{\mathfrak{A}_{\phi}}, \delta h_{\mathfrak{A}_{\phi}}\right) \\
& -S^{A}\left(f_{\mathfrak{A}_{\phi}}\right) G_{0}^{A}\left(1, g_{\mathfrak{A}_{\phi}}\right) H_{0}^{A}\left(1, \delta h_{\mathfrak{A}_{\phi}}\right) \\
& +\sum_{m=1}^{n-1}\left[W_{K}^{A}\left(F_{\mathfrak{A}_{\phi}}^{[m]}, f_{\mathfrak{A}_{\phi}}\right) G_{0}^{A}\left(G_{\mathfrak{A}_{\phi}}^{[m]}, g_{\mathfrak{A}_{\phi}}\right) H_{0}^{A}\left(H_{\mathfrak{A}_{\phi}}^{[m]}, \delta h_{\mathfrak{A}_{\phi}}\right)\right. \\
& \left.+\mathrm{i} W_{C}^{A}\left(F_{\mathfrak{A}_{\phi}}^{[m]}, f_{\mathfrak{A}_{\phi}}\right) G_{1}^{A}\left(G_{\mathfrak{A}_{\phi}}^{[m]}, g_{\mathfrak{A}_{\phi}}\right) H_{1}^{A}\left(H_{\mathfrak{A}_{\phi}}^{[m]}, \delta h_{\mathfrak{A}_{\phi}}\right)\right],
\end{aligned}
$$


where the terms $G_{0}^{A}, G_{1}^{A}, H_{0}^{A}, H_{1}^{A}$ associated with the parametric domain $\Omega_{q}$ are generically defined in terms of the fields $b, c$ as

$$
\begin{aligned}
G_{0}^{a}(b, c):=\int_{\Omega_{\omega}^{a}} b \bar{c} \mathrm{~d} \omega, \quad G_{1}^{a}(b, c):=\int_{\Omega_{\omega}^{a}} b \bar{c} \omega \mathrm{d} \omega \\
H_{0}^{a}(b, c):=\int_{\Omega_{\gamma}^{a}} b \bar{c} \mathrm{~d} \gamma, \quad H_{1}^{a}(b, c):=\int_{\Omega_{\gamma}^{a}} b \bar{c} \alpha_{\gamma} \mathrm{d} \gamma,
\end{aligned}
$$

where the upper index $(\cdot)^{a}$ defines the domain of integration (i.e. $(\cdot)^{A}$ for electromagnetics or $(\cdot)^{u}$ for mechanics). The high-dimensional electromagnetic problem (25) is solved for the solution fields increments as

$$
f_{\mathfrak{A}_{\phi}}^{[k+1]}:=f_{\mathfrak{A}_{\phi}}^{[k]}+\Delta f_{\mathfrak{A}_{\phi}}, \quad g_{\mathfrak{A}_{\phi}}^{[k+1]}:=g_{\mathfrak{A}_{\phi}}^{[k]}+\Delta g_{\mathfrak{A}_{\phi}}, \quad h_{\mathfrak{A}_{\phi}}^{[k+1]}:=h_{\mathfrak{A}_{\phi}}^{[k]}+\Delta h_{\mathfrak{A}_{\phi}},
$$

where the concept of directional derivatives [40] is used to formulate the problem as: Find $\left(\Delta f_{\mathfrak{A}_{\phi}}, \Delta g_{\mathfrak{A}_{\phi}}, \Delta h_{\mathfrak{A}_{\phi}}\right) \in X(0) \times Z\left(\Omega_{\omega}^{A}\right) \times Z\left(\Omega_{\gamma}^{A}\right)$ such that

$$
\begin{aligned}
& D \mathcal{R}_{f_{\mathfrak{A}_{\phi}}}\left(\delta f_{\mathfrak{A}_{\phi}} ; \mathcal{A}_{\phi}^{n-1}, f_{\mathfrak{A}_{\phi}}, g_{\mathfrak{A}_{\phi}}, h_{\mathfrak{A}_{\phi}}\right)\left[\Delta f_{\mathfrak{A}_{\phi}}\right]=-\mathcal{R}_{f_{\mathfrak{A}_{\phi}}}\left(\delta f_{\mathfrak{A}_{\phi}} ; \mathcal{A}_{\phi}^{n-1}, f_{\mathfrak{A}_{\phi}}, g_{\mathfrak{A}_{\phi}}, h_{\mathfrak{A}_{\phi}}\right), \\
& D \mathcal{R}_{g_{\mathfrak{A}_{\phi}}}\left(\delta g_{\mathfrak{A}_{\phi}} ; \mathcal{A}_{\phi}^{n-1}, f_{\mathfrak{A}_{\phi}}, g_{\mathfrak{A}_{\phi}}, h_{\mathfrak{A}_{\phi}}\right)\left[\Delta g_{\mathfrak{A}_{\phi}}\right]=-\mathcal{R}_{g_{\mathfrak{A}_{\phi}}}\left(\delta g_{\mathfrak{A}_{\phi}} ; \mathcal{A}_{\phi}^{n-1}, f_{\mathfrak{A}_{\phi}}, g_{\mathfrak{A}_{\phi}}, h_{\mathfrak{A}_{\phi}}\right), \\
& D \mathcal{R}_{h_{\mathfrak{A}_{\phi}}}\left(\delta h_{\mathfrak{A}_{\phi}} ; \mathcal{A}_{\phi}^{n-1}, f_{\mathfrak{A}_{\phi}}, g_{\mathfrak{A}_{\phi}}, h_{\mathfrak{A}_{\phi}}\right)\left[\Delta h_{\mathfrak{A}_{\phi}}\right]=-\mathcal{R}_{h_{\mathfrak{A}_{\phi}}}\left(\delta h_{\mathfrak{A}_{\phi}} ; \mathcal{A}_{\phi}^{n-1}, f_{\mathfrak{A}_{\phi}}, g_{\mathfrak{A}_{\phi}}, h_{\mathfrak{A}_{\phi}}\right),
\end{aligned}
$$

$\forall\left(\delta f_{\mathfrak{A}_{\phi}}, \delta g_{\mathfrak{A}_{\phi}}, \delta h_{\mathfrak{A}_{\phi}}\right) \in X(0) \times Z\left(\Omega_{\omega}^{A}\right) \times Z\left(\Omega_{\gamma}^{A}\right)$, where the directional derivatives are defined as

$$
\begin{aligned}
D \mathcal{R}_{f_{\mathfrak{A}_{\phi}}}\left(\delta f_{\mathfrak{A}_{\phi}} ; \mathcal{A}_{\phi}^{n-1}, f_{\mathfrak{A}_{\phi}}, g_{\mathfrak{A}_{\phi}}, h_{\mathfrak{A}_{\phi}}\right)\left[\Delta f_{\mathfrak{A}_{\phi}}\right] & =W_{K}^{A}\left(\Delta f_{\mathfrak{A}_{\phi}}, \delta f_{\mathfrak{A}_{\phi}}\right) G_{0}^{A}\left(g_{\mathfrak{A}_{\phi}}, g_{\mathfrak{A}_{\phi}}\right) H_{0}^{A}\left(h_{\mathfrak{A}_{\phi}}, h_{\mathfrak{A}_{\phi}}\right) \\
& +\mathrm{i} W_{C}^{A}\left(\Delta f_{\mathfrak{A}_{\phi}}, \delta f_{\mathfrak{A}_{\phi}}\right) G_{1}^{A}\left(g_{\mathfrak{A}_{\phi}}, g_{\mathfrak{A}_{\phi}}\right) H_{1}^{A}\left(h_{\mathfrak{A}_{\phi}}, h_{\mathfrak{A}_{\phi}}\right), \\
D \mathcal{R}_{g_{\mathfrak{A}_{\phi}}}\left(\delta g_{\mathfrak{A}_{\phi}} ; \mathcal{A}_{\phi}^{n-1}, f_{\mathfrak{A}_{\phi}}, g_{\mathfrak{A}_{\phi}}, h_{\mathfrak{A}_{\phi}}\right)\left[\Delta g_{\mathfrak{A}_{\phi}}\right] & =W_{K}^{A}\left(f_{\mathfrak{A}_{\phi}}, f_{\mathfrak{A}_{\phi}}\right) G_{0}^{A}\left(\Delta g_{\mathfrak{A}_{\phi}}, \delta g_{\mathfrak{A}_{\phi}}\right) H_{0}^{A}\left(h_{\mathfrak{A}_{\phi}}, h_{\mathfrak{A}_{\phi}}\right) \\
& +\mathrm{i} W_{C}^{A}\left(f_{\mathfrak{A}_{\phi}}, f_{\mathfrak{A}_{\phi}}\right) G_{1}^{A}\left(\Delta g_{\mathfrak{A}_{\phi}}, \delta g_{\mathfrak{A}_{\phi}}\right) H_{1}^{A}\left(h_{\mathfrak{A}_{\phi}}, h_{\mathfrak{A}_{\phi}}\right), \\
D \mathcal{R}_{h_{\mathfrak{A}_{\phi}}}\left(\delta h_{\mathfrak{A}_{\phi}} ; \mathcal{A}_{\phi}^{n-1}, f_{\mathfrak{A}_{\phi}}, g_{\mathfrak{A}_{\phi}}, h_{\mathfrak{A}_{\phi}}\right)\left[\Delta h_{\mathfrak{A}_{\phi}}\right] & =W_{K}^{A}\left(f_{\mathfrak{A}_{\phi}}, f_{\mathfrak{A}_{\phi}}\right) G_{0}^{A}\left(g_{\mathfrak{A}_{\phi}}, g_{\mathfrak{A}_{\phi}}\right) H_{0}^{A}\left(\Delta h_{\mathfrak{A}_{\phi}}, \delta h_{\mathfrak{A}_{\phi}}\right) \\
& +\mathrm{i} W_{C}^{A}\left(f_{\mathfrak{A}_{\phi}}, f_{\mathfrak{A}_{\phi}}\right) G_{1}^{A}\left(g_{\mathfrak{A}_{\phi}}, g_{\mathfrak{A}_{\phi}}\right) H_{1}^{A}\left(\Delta h_{\mathfrak{A}_{\phi}}, \delta h_{\mathfrak{A}_{\phi}}\right) .
\end{aligned}
$$

The standard Galerkin FEM discretisation [11] is carried out in order to numerically solve the described electromagnetic problem. The global discretised version of (A.4) is obtained by the standard assembling procedure [11] as

$$
\begin{aligned}
& \left(g_{0}^{A} h_{0}^{A} \mathbf{K}^{A}+\mathrm{i} g_{1}^{A} h_{1}^{A} \mathbf{C}^{A}\right) \Delta \mathbf{f}_{\mathcal{A}_{\phi}}=-\mathbf{R}_{\mathfrak{A}_{\phi}}\left(f_{\mathfrak{A}_{\phi}}, g_{\mathfrak{A}_{\phi}}, h_{\mathfrak{A}_{\phi}}\right), \\
& \left(k^{A} h_{0}^{A} \mathbf{G}_{0}^{A}+\mathrm{i} c^{A} h_{1}^{A} \mathbf{G}_{1}^{A}\right) \Delta \mathbf{g}_{\mathfrak{A}_{\phi}}=-\mathbf{R}_{\mathfrak{A}_{\mathfrak{A}_{\phi}}}\left(f_{\mathfrak{A}_{\phi}}, g_{\mathfrak{A}_{\phi}}, h_{\mathfrak{A}_{\phi}}\right), \\
& \left(k^{A} g_{0}^{A} \mathbf{H}_{0}^{A}+\mathrm{i} c^{A} g_{1}^{A} \mathbf{H}_{1}^{A}\right) \Delta \mathbf{h}_{\mathcal{A}_{\phi}}=-\mathbf{R}_{h_{\mathfrak{A}_{\phi}}}\left(f_{\mathfrak{A}_{\phi}}, g_{\mathfrak{A}_{\phi}}, h_{\mathfrak{A}_{\phi}}\right),
\end{aligned}
$$

where the Roman (non-italic) font denotes a certain discretised quantity. The discretised version of the residuals is

$$
\begin{aligned}
\mathbf{R}_{f_{\mathfrak{A}_{\phi}}}\left(f_{\mathcal{A}_{\phi}}, g_{\mathfrak{A}_{\phi}}, h_{\mathcal{A}_{\phi}}\right) & =\left(g_{0}^{A} h_{0}^{A} \mathbf{K}^{A}+\mathrm{i} g_{1}^{A} h_{1}^{A} \mathbf{C}^{A}\right) \mathbf{f}_{\mathcal{A}_{\phi}}-g_{0}^{A, s} h_{0}^{A, s} \mathbf{s}^{A} \\
& +\sum_{m=1}^{n-1}\left[g_{0}^{A,[m]} h_{0}^{A,[m]} \mathbf{K}^{A}+\mathrm{i} g_{1}^{A,[m]} h_{1}^{A,[m]} \mathbf{C}^{A}\right] \mathbf{F}_{\mathcal{A}_{\phi}}^{[m]}, \\
\mathbf{R}_{\mathfrak{A}_{\mathfrak{A}_{\phi}}}\left(f_{\mathcal{A}_{\phi}}, g_{\mathfrak{A}_{\phi}}, h_{\mathfrak{A}_{\phi}}\right) & =\left(k^{A} h_{0}^{A} \mathbf{G}_{0}^{A}+\mathrm{i} c^{A} h_{1}^{A} \mathbf{G}_{1}^{A}\right) \mathbf{g}_{\mathfrak{A}_{\phi}}-s^{A} h_{0}^{A, s} \mathbf{g}_{0}^{A, s} \\
& +\sum_{m=1}^{n-1}\left[k^{A,[m]} h_{0}^{A,[m]} \mathbf{G}_{0}^{A}+\mathrm{i} c^{A,[m]} h_{1}^{A,[m]} \mathbf{G}_{1}^{A}\right] \mathbf{G}_{\mathcal{A}_{\phi}}^{[m]},
\end{aligned}
$$




$$
\begin{aligned}
\mathbf{R}_{h_{\mathfrak{A}_{\phi}}}\left(f_{\mathfrak{A}_{\phi}}, g_{\mathfrak{A}_{\phi}}, h_{\mathfrak{A}_{\phi}}\right) & =\left(k^{A} g_{0}^{A} \mathbf{H}_{0}^{A}+\mathrm{i} c^{A} g_{1}^{A} \mathbf{H}_{1}^{A}\right) \mathbf{h}_{\mathfrak{A}_{\phi}}-s^{A} g_{0}^{A, s} \mathbf{h}_{0}^{A, s} \\
& +\sum_{m=1}^{n-1}\left[k^{A,[m]} g_{0}^{A,[m]} \mathbf{H}_{0}^{A A}+\mathrm{i} c^{A,[m]} g_{1}^{A,[m]} \mathbf{H}_{1}^{A}\right] \mathbf{H}_{\mathcal{A}_{\phi}}^{[m]}
\end{aligned}
$$

and where the scalar quantities are defined as

$$
\begin{aligned}
& g_{0}^{A}:=G_{0}^{A}\left(g_{\mathfrak{A}_{\phi}}, g_{\mathfrak{A}_{\phi}}\right), \quad h_{0}^{A}:=H_{0}^{A}\left(h_{\mathfrak{A}_{\phi}}, h_{\mathfrak{A}_{\phi}}\right), \quad k^{A}:=W_{K}^{A}\left(f_{\mathfrak{A}_{\phi}}, f_{\mathfrak{A}_{\phi}}\right), \\
& g_{1}^{A}:=G_{1}^{A}\left(g_{\mathfrak{A}_{\phi}}, g_{\mathfrak{A}_{\phi}}\right), \quad h_{1}^{A}:=H_{1}^{A}\left(h_{\mathfrak{A}_{\phi}}, h_{\mathfrak{A}_{\phi}}\right), \quad c^{A}:=W_{C}^{A}\left(f_{\mathfrak{A}_{\phi}}, f_{\mathfrak{A}_{\phi}}\right), \\
& g_{0}^{A, s}:=G_{0}^{A}\left(1, g_{\mathfrak{A}_{\phi}}\right), \quad h_{0}^{A, s}:=H_{0}^{A}\left(1, h_{\mathfrak{A}_{\phi}}\right), \quad s^{A}:=S^{A}\left(1, f_{\mathfrak{A}_{\phi}}\right), \\
& g_{0}^{A,[m]}:=G_{0}^{A}\left(G_{\mathfrak{A}_{\phi}}^{[m]}, g_{\mathfrak{A}_{\phi}}\right), \quad h_{0}^{A,[m]}:=H_{0}^{A}\left(H_{\mathfrak{A}_{\phi}}^{[m]}, h_{\mathfrak{A}_{\phi}}\right), \quad k^{A,[m]}:=W_{K}^{A}\left(F_{\mathfrak{A}_{\phi}}^{[m]}, f_{\mathfrak{A}_{\phi}}\right), \\
& g_{1}^{A,[m]}:=G_{1}^{A}\left(G_{\mathfrak{A}_{\phi}}^{[m]}, g_{\mathfrak{A}_{\phi}}\right), \quad h_{1}^{A,[m]}:=H_{1}^{A}\left(H_{\mathfrak{A}_{\phi}}^{[m]}, h_{\mathfrak{A}_{\phi}}\right), \quad c^{A,[m]}:=W_{C}^{A}\left(F_{\mathfrak{A}_{\phi}}^{[m]}, f_{\mathfrak{A}_{\phi}}\right),
\end{aligned}
$$

\section{Appendix A.2. Mechanics}

The mechanical residual forms defined in (31) can be expanded by using the separability introduced in (28). The separated expression of these residuals are

$$
\begin{aligned}
\mathcal{R}_{\boldsymbol{f}_{\boldsymbol{u}}}\left(\delta \boldsymbol{f}_{\boldsymbol{u}} ; \boldsymbol{u}^{n-1}, \boldsymbol{f}_{\boldsymbol{u}}, g_{\boldsymbol{u}}, h_{\boldsymbol{u}}, l_{\boldsymbol{u}}\right) & =W_{K}^{u}\left(\boldsymbol{f}_{\boldsymbol{u}}, \delta \boldsymbol{f}_{\boldsymbol{u}}\right) G_{0}^{u}\left(g_{\boldsymbol{u}}, g_{\boldsymbol{u}}\right) H_{0}^{u}\left(h_{\boldsymbol{u}}, h_{\boldsymbol{u}}\right) L_{0}^{u}\left(l_{\boldsymbol{u}}, l_{\boldsymbol{u}}\right) \\
& +\mathrm{i} W_{C}^{u}\left(\boldsymbol{f}_{\boldsymbol{u}}, \delta \boldsymbol{f}_{\boldsymbol{u}}\right) G_{2}^{u}\left(g_{\boldsymbol{u}}, g_{\boldsymbol{u}}\right) H_{0}^{u}\left(h_{\boldsymbol{u}}, h_{\boldsymbol{u}}\right) L_{0}^{u}\left(l_{\boldsymbol{u}}, l_{\boldsymbol{u}}\right) \\
& -W_{M}^{u}\left(\boldsymbol{f}_{\boldsymbol{u}}, \delta \boldsymbol{f}_{\boldsymbol{u}}\right) G_{2}^{u}\left(g_{\boldsymbol{u}}, g_{\boldsymbol{u}}\right) H_{0}^{u}\left(h_{\boldsymbol{u}}, h_{\boldsymbol{u}}\right) L_{0}^{u}\left(l_{\boldsymbol{u}}, l_{\boldsymbol{u}}\right) \\
& +\sum_{m=1}^{N_{A}}\left[S^{u}\left(F_{\mathfrak{A}_{\phi}}^{[m]}, \delta \boldsymbol{f}_{\boldsymbol{u}}\right) G_{0}^{u}\left(G_{\mathcal{A}_{\phi}}^{[m]}, g_{\boldsymbol{u}}\right) H_{0}^{u}\left(H_{\mathcal{A}_{\phi}}^{[m]}, h_{\boldsymbol{u}}\right) L_{1}^{u}\left(L_{\mathfrak{A}_{\phi}}^{[m]}, l_{\boldsymbol{u}}\right)\right] \\
& +\sum_{m=1}^{n-1}\left[W_{K}^{u}\left(\boldsymbol{F}_{\boldsymbol{u}}^{[m]}, \delta \boldsymbol{f}_{\boldsymbol{u}}\right) G_{0}^{u}\left(G_{\boldsymbol{u}}^{[m]}, g_{\boldsymbol{u}}\right) H_{0}^{u}\left(H_{\boldsymbol{u}}^{[m]}, h_{\boldsymbol{u}}\right) L_{0}^{u}\left(L_{\boldsymbol{u}}^{[m]}, l_{\boldsymbol{u}}\right)\right. \\
& +\mathrm{i} W_{K}^{u}\left(\boldsymbol{F}_{\boldsymbol{u}}^{[m]}, \delta \boldsymbol{f}_{\boldsymbol{u}}\right) G_{2}^{u}\left(G_{\boldsymbol{u}}^{[m]}, g_{\boldsymbol{u}}\right) H_{0}^{u}\left(H_{\boldsymbol{u}}^{[m]}, h_{\boldsymbol{u}}\right) L_{0}^{u}\left(L_{\boldsymbol{u}}^{[m]}, l_{\boldsymbol{u}}\right) \\
& \left.-W_{K}^{u}\left(\boldsymbol{F}_{\boldsymbol{u}}^{[m]}, \delta \boldsymbol{f}_{\boldsymbol{u}}\right) G_{2}^{u}\left(G_{\boldsymbol{u}}^{[m]}, g_{\boldsymbol{u}}\right) H_{0}^{u}\left(H_{\boldsymbol{u}}^{[m]}, h_{\boldsymbol{u}}\right) L_{0}^{u}\left(L_{\boldsymbol{u}}^{[m]}, l_{\boldsymbol{u}}\right)\right],
\end{aligned}
$$

$$
\begin{aligned}
\mathcal{R}_{g_{\boldsymbol{u}}}\left(\delta g_{\boldsymbol{u}} ; \boldsymbol{u}^{n-1}, \boldsymbol{f}_{\boldsymbol{u}}, g_{\boldsymbol{u}}, h_{\boldsymbol{u}}, l_{\boldsymbol{u}}\right) & =W_{K}^{u}\left(\boldsymbol{f}_{\boldsymbol{u}}, \boldsymbol{f}_{\boldsymbol{u}}\right) G_{0}^{u}\left(g_{\boldsymbol{u}}, \delta g_{\boldsymbol{u}}\right) H_{0}^{u}\left(h_{\boldsymbol{u}}, h_{\boldsymbol{u}}\right) L_{0}^{u}\left(l_{\boldsymbol{u}}, l_{\boldsymbol{u}}\right) \\
& +\mathrm{i} W_{C}^{u}\left(\boldsymbol{f}_{\boldsymbol{u}}, \boldsymbol{f}_{\boldsymbol{u}}\right) G_{2}^{u}\left(g_{\boldsymbol{u}}, \delta g_{\boldsymbol{u}}\right) H_{0}^{u}\left(h_{\boldsymbol{u}}, h_{\boldsymbol{u}}\right) L_{0}^{u}\left(l_{\boldsymbol{u}}, l_{\boldsymbol{u}}\right) \\
& -W_{M}^{u}\left(\boldsymbol{f}_{\boldsymbol{u}}, \boldsymbol{f}_{\boldsymbol{u}}\right) G_{2}^{u}\left(g_{\boldsymbol{u}}, \delta g_{\boldsymbol{u}}\right) H_{0}^{u}\left(h_{\boldsymbol{u}}, h_{\boldsymbol{u}}\right) L_{0}^{u}\left(l_{\boldsymbol{u}}, l_{\boldsymbol{u}}\right) \\
& +\sum_{m=1}^{N_{A}}\left[S^{u}\left(F_{\mathfrak{A}_{\phi}}^{[m]}, \boldsymbol{f}_{\boldsymbol{u}}\right) G_{0}^{u}\left(G_{\mathcal{A}_{\phi}}^{[m]}, \delta g_{\boldsymbol{u}}\right) H_{0}^{u}\left(H_{\mathcal{A}_{\phi}}^{[m]}, h_{\boldsymbol{u}}\right) L_{1}^{u}\left(L_{\left.\mathcal{A}_{\phi}\right]}^{[m]}, l_{\boldsymbol{u}}\right)\right] \\
& +\sum_{m=1}^{n-1}\left[W_{K}^{u}\left(\boldsymbol{F}_{\boldsymbol{u}}^{[m]}, \boldsymbol{f}_{\boldsymbol{u}}\right) G_{0}^{u}\left(G_{\boldsymbol{u}}^{[m]}, \delta g_{\boldsymbol{u}}\right) H_{0}^{u}\left(H_{\boldsymbol{u}}^{[m]}, h_{\boldsymbol{u}}\right) L_{0}^{u}\left(L_{\boldsymbol{u}}^{[m]}, l_{\boldsymbol{u}}\right)\right. \\
& +\mathrm{i} W_{K}^{u}\left(\boldsymbol{F}_{\boldsymbol{u}}^{[m]}, \boldsymbol{f}_{\boldsymbol{u}}\right) G_{2}^{u}\left(G_{\boldsymbol{u}}^{[m]}, \delta g_{\boldsymbol{u}}\right) H_{0}^{u}\left(H_{\boldsymbol{u}}^{[m]}, h_{\boldsymbol{u}}\right) L_{0}^{u}\left(L_{\boldsymbol{u}}^{[m]}, l_{\boldsymbol{u}}\right) \\
& \left.-W_{K}^{u}\left(\boldsymbol{F}_{\boldsymbol{u}}^{[m]}, \boldsymbol{f}_{\boldsymbol{u}}\right) G_{2}^{u}\left(G_{\boldsymbol{u}}^{[m]}, \delta g_{\boldsymbol{u}}\right) H_{0}^{u}\left(H_{\boldsymbol{u}}^{[m]}, h_{\boldsymbol{u}}\right) L_{0}^{u}\left(L_{\boldsymbol{u}}^{[m]}, l_{\boldsymbol{u}}\right)\right]
\end{aligned}
$$




$$
\begin{aligned}
\mathcal{R}_{h_{\mathfrak{u}}}\left(\delta h_{\boldsymbol{u}} ; \boldsymbol{u}^{n-1}, \boldsymbol{f}_{\boldsymbol{u}}, g_{\boldsymbol{u}}, h_{\boldsymbol{u}}, l_{\boldsymbol{u}}\right) & =W_{K}^{u}\left(\boldsymbol{f}_{\boldsymbol{u}}, \boldsymbol{f}_{\boldsymbol{u}}\right) G_{0}^{u}\left(g_{\boldsymbol{u}}, g_{\boldsymbol{u}}\right) H_{0}^{u}\left(h_{\boldsymbol{u}}, \delta h_{\boldsymbol{u}}\right) L_{0}^{u}\left(l_{\boldsymbol{u}}, l_{\boldsymbol{u}}\right) \\
& +\mathrm{i} W_{C}^{u}\left(\boldsymbol{f}_{\boldsymbol{u}}, \boldsymbol{f}_{\boldsymbol{u}}\right) G_{2}^{u}\left(g_{\boldsymbol{u}}, g_{\boldsymbol{u}}\right) H_{0}^{u}\left(h_{\boldsymbol{u}}, \delta h_{\boldsymbol{u}}\right) L_{0}^{u}\left(l_{\boldsymbol{u}}, l_{\boldsymbol{u}}\right) \\
& -W_{M}^{u}\left(\boldsymbol{f}_{\boldsymbol{u}}, \boldsymbol{f}_{\boldsymbol{u}}\right) G_{2}^{u}\left(g_{\boldsymbol{u}}, g_{\boldsymbol{u}}\right) H_{0}^{u}\left(h_{\boldsymbol{u}}, \delta h_{\boldsymbol{u}}\right) L_{0}^{u}\left(l_{\boldsymbol{u}}, l_{\boldsymbol{u}}\right) \\
& +\sum_{m=1}^{N_{A}}\left[S^{u}\left(F_{\mathfrak{A}_{\phi}}^{[m]}, \boldsymbol{f}_{\boldsymbol{u}}\right) G_{0}^{u}\left(G_{\mathcal{A}_{\phi}}^{[m]}, g_{\boldsymbol{u}}\right) H_{0}^{u}\left(H_{\mathcal{A}_{\phi}}^{[m]}, \delta h_{\boldsymbol{u}}\right) L_{1}^{u}\left(L_{\mathcal{A}_{\phi}}^{[m]}, l_{\boldsymbol{u}}\right)\right] \\
& +\sum_{m=1}^{n-1}\left[W_{K}^{u}\left(\boldsymbol{F}_{\boldsymbol{u}}^{[m]}, \boldsymbol{f}_{\boldsymbol{u}}\right) G_{0}^{u}\left(G_{\boldsymbol{u}}^{[m]}, g_{\boldsymbol{u}}\right) H_{0}^{u}\left(H_{\boldsymbol{u}}^{[m]}, \delta h_{\boldsymbol{u}}\right) L_{0}^{u}\left(L_{\boldsymbol{u}}^{[m]}, l_{\boldsymbol{u}}\right)\right. \\
& +\mathrm{i} W_{K}^{u}\left(\boldsymbol{F}_{\boldsymbol{u}}^{[m]}, \boldsymbol{f}_{\boldsymbol{u}}\right) G_{2}^{u}\left(G_{\boldsymbol{u}}^{[m]}, g_{\boldsymbol{u}}\right) H_{0}^{u}\left(H_{\boldsymbol{u}}^{[m]}, \delta h_{\boldsymbol{u}}\right) L_{0}^{u}\left(L_{\boldsymbol{u}}^{[m]}, l_{\boldsymbol{u}}\right) \\
& \left.-W_{K}^{u}\left(\boldsymbol{F}_{\boldsymbol{u}}^{[m]}, \boldsymbol{f}_{\boldsymbol{u}}\right) G_{2}^{u}\left(G_{\boldsymbol{u}}^{[m]}, g_{\boldsymbol{u}}\right) H_{0}^{u}\left(H_{\boldsymbol{u}}^{[m]}, \delta h_{\boldsymbol{u}}\right) L_{0}^{u}\left(L_{\boldsymbol{u}}^{[m]}, l_{\boldsymbol{u}}\right)\right],
\end{aligned}
$$

$$
\begin{aligned}
\mathcal{R}_{l_{\boldsymbol{u}}}\left(\delta l_{\boldsymbol{u}} ; \boldsymbol{u}^{n-1}, \boldsymbol{f}_{\boldsymbol{u}}, g_{\boldsymbol{u}}, h_{\boldsymbol{u}}, l_{\boldsymbol{u}}\right) & =W_{K}^{u}\left(\boldsymbol{f}_{\boldsymbol{u}}, \boldsymbol{f}_{\boldsymbol{u}}\right) G_{0}^{u}\left(g_{\boldsymbol{u}}, g_{\boldsymbol{u}}\right) H_{0}^{u}\left(h_{\boldsymbol{u}}, h_{\boldsymbol{u}}\right) L_{0}^{u}\left(l_{\boldsymbol{u}}, \delta l_{\boldsymbol{u}}\right) \\
& +\mathrm{i} W_{C}^{u}\left(\boldsymbol{f}_{\boldsymbol{u}}, \boldsymbol{f}_{\boldsymbol{u}}\right) G_{2}^{u}\left(g_{\boldsymbol{u}}, g_{\boldsymbol{u}}\right) H_{0}^{u}\left(h_{\boldsymbol{u}}, h_{\boldsymbol{u}}\right) L_{0}^{u}\left(l_{\boldsymbol{u}}, \delta l_{\boldsymbol{u}}\right) \\
& -W_{M}^{u}\left(\boldsymbol{f}_{\boldsymbol{u}}, \boldsymbol{f}_{\boldsymbol{u}}\right) G_{2}^{u}\left(g_{\boldsymbol{u}}, g_{\boldsymbol{u}}\right) H_{0}^{u}\left(h_{\boldsymbol{u}}, h_{\boldsymbol{u}}\right) L_{0}^{u}\left(l_{\boldsymbol{u}}, \delta l_{\boldsymbol{u}}\right) \\
& +\sum_{m=1}^{N_{A}}\left[S^{u}\left(F_{\mathfrak{A}_{\phi}}^{[m]}, \boldsymbol{f}_{\boldsymbol{u}}\right) G_{0}^{u}\left(G_{\mathcal{A}_{\phi}}^{[m]}, g_{\boldsymbol{u}}\right) H_{0}^{u}\left(H_{\mathfrak{A}_{\phi}}^{[m]}, h_{\boldsymbol{u}}\right) L_{1}^{u}\left(L_{\boldsymbol{A}_{\phi}}^{[m]}, \delta l_{\boldsymbol{u}}\right)\right] \\
& +\sum_{m=1}^{n-1}\left[W_{K}^{u}\left(\boldsymbol{F}_{\boldsymbol{u}}^{[m]}, \boldsymbol{f}_{\boldsymbol{u}}\right) G_{0}^{u}\left(G_{\boldsymbol{u}}^{[m]}, g_{\boldsymbol{u}}\right) H_{0}^{u}\left(H_{\boldsymbol{u}}^{[m]}, h_{\boldsymbol{u}}\right) L_{0}^{u}\left(L_{\boldsymbol{u}}^{[m]}, \delta l_{\boldsymbol{u}}\right)\right. \\
& +\mathrm{i} W_{K}^{u}\left(\boldsymbol{F}_{\boldsymbol{u}}^{[m]}, \boldsymbol{f}_{\boldsymbol{u}}\right) G_{2}^{u}\left(G_{\boldsymbol{u}}^{[m]}, g_{\boldsymbol{u}}\right) H_{0}^{u}\left(H_{\boldsymbol{u}}^{[m]}, h_{\boldsymbol{u}}\right) L_{0}^{u}\left(L_{\boldsymbol{u}}^{[m]}, \delta l_{\boldsymbol{u}}\right) \\
& \left.-W_{K}^{u}\left(\boldsymbol{F}_{\boldsymbol{u}}^{[m]}, \boldsymbol{f}_{\boldsymbol{u}}\right) G_{2}^{u}\left(G_{\boldsymbol{u}}^{[m]}, g_{\boldsymbol{u}}\right) H_{0}^{u}\left(H_{\boldsymbol{u}}^{[m]}, h_{\boldsymbol{u}}\right) L_{0}^{u}\left(L_{\boldsymbol{u}}^{[m]}, \delta l_{\boldsymbol{u}}\right)\right] .
\end{aligned}
$$

where the terms related to the parametric domain $\Omega_{q}$ are defined following the same notation than in (A.2) as

$$
\begin{aligned}
G_{2}^{a}(b, c) & :=\int_{\Omega_{\omega}^{a}} b \bar{c} \omega^{2} \mathrm{~d} \omega \\
L_{0}^{a}(b, c) & :=\int_{\Omega_{B_{0}}^{a}} b \bar{c} \mathrm{~d} B_{0}, \quad L_{1}^{a}(b, c):=\int_{\Omega_{B_{0}}^{a}} b \bar{c} \alpha_{B_{0}} \mathrm{~d} B_{0} .
\end{aligned}
$$

The high-dimensional mechanical problem will be solved incrementally as

$$
\begin{gathered}
\boldsymbol{f}_{\boldsymbol{u}}^{[k+1]}:=\boldsymbol{f}_{\boldsymbol{u}}^{[k]}+\Delta \boldsymbol{f}_{\boldsymbol{u}}, \quad g_{\boldsymbol{u}}^{[k+1]}:=g_{\boldsymbol{u}}^{[k]}+\Delta g_{\boldsymbol{u}}, \\
h_{\boldsymbol{u}}^{[k+1]}:=h_{\mathfrak{u}}^{[k]}+\Delta h_{\boldsymbol{u}}, \quad l_{\boldsymbol{u}}^{[k+1]}:=l_{\boldsymbol{u}}^{[k]}+\Delta l_{\boldsymbol{u}},
\end{gathered}
$$

where the directional derivative definition [40] is used to formulate the problem as: Find $\left(\Delta \boldsymbol{f}_{\boldsymbol{u}}, \Delta g_{\boldsymbol{u}}, \Delta h_{\boldsymbol{u}}, \Delta l_{\boldsymbol{u}}\right) \in X(0) \times Z\left(\Omega_{\omega}^{u}\right) \times Z\left(\Omega_{\gamma}^{u}\right) \times Z\left(\Omega_{B_{0}}^{u}\right)$ such that

$$
\begin{aligned}
D \mathcal{R}_{\boldsymbol{f}_{\boldsymbol{u}}}\left(\delta \boldsymbol{f}_{\boldsymbol{u}} ; \boldsymbol{u}^{n-1}, \boldsymbol{f}_{\boldsymbol{u}}, g_{\boldsymbol{u}}, h_{\boldsymbol{u}}, l_{\boldsymbol{u}}\right)\left[\Delta \boldsymbol{f}_{\boldsymbol{u}}\right] & =-\mathcal{R}_{\boldsymbol{f}_{\boldsymbol{u}}}\left(\delta \boldsymbol{f}_{\boldsymbol{u}} ; \boldsymbol{u}^{n-1}, \boldsymbol{f}_{\boldsymbol{u}}, g_{\boldsymbol{u}}, h_{\boldsymbol{u}}, l_{\boldsymbol{u}}\right), \\
D \mathcal{R}_{g_{\boldsymbol{u}}}\left(\delta g_{\boldsymbol{u}} ; \boldsymbol{u}^{n-1}, \boldsymbol{f}_{\boldsymbol{u}}, g_{\boldsymbol{u}}, h_{\boldsymbol{u}}, l_{\boldsymbol{u}}\right)\left[\Delta g_{\boldsymbol{u}}\right] & =-\mathcal{R}_{g_{\boldsymbol{u}}}\left(\delta g_{\boldsymbol{u}} ; \boldsymbol{u}^{n-1}, \boldsymbol{f}_{\boldsymbol{u}}, g_{\boldsymbol{u}}, h_{\boldsymbol{u}}, l_{\boldsymbol{u}}\right), \\
D \mathcal{R}_{h_{\boldsymbol{u}}}\left(\delta h_{\boldsymbol{u}} ; \boldsymbol{u}^{n-1}, \boldsymbol{f}_{\boldsymbol{u}}, g_{\boldsymbol{u}}, h_{\boldsymbol{u}}, l_{\boldsymbol{u}}\right)\left[\Delta h_{\boldsymbol{u}}\right] & =-\mathcal{R}_{h_{\boldsymbol{u}}}\left(\delta h_{\boldsymbol{u}} ; \boldsymbol{u}^{n-1}, \boldsymbol{f}_{\boldsymbol{u}}, g_{\boldsymbol{u}}, h_{\boldsymbol{u}}, l_{\boldsymbol{u}}\right), \\
D \mathcal{R}_{l_{\boldsymbol{u}}}\left(\delta l_{\boldsymbol{u}} ; \boldsymbol{u}^{n-1}, \boldsymbol{f}_{\boldsymbol{u}}, g_{\boldsymbol{u}}, h_{\boldsymbol{u}}, l_{\boldsymbol{u}}\right)\left[\Delta l_{\boldsymbol{u}}\right] & =-\mathcal{R}_{l_{\boldsymbol{u}}}\left(\delta l_{\boldsymbol{u}} ; \boldsymbol{u}^{n-1}, \boldsymbol{f}_{\boldsymbol{u}}, g_{\boldsymbol{u}}, h_{\boldsymbol{u}}, l_{\boldsymbol{u}}\right),
\end{aligned}
$$

$\forall\left(\delta \boldsymbol{f}_{\boldsymbol{u}}, \delta g_{\boldsymbol{u}}, \delta h_{\boldsymbol{u}}, \delta l_{\boldsymbol{u}}\right) \in X(0) \times Z\left(\Omega_{\omega}^{u}\right) \times Z\left(\Omega_{\gamma}^{u}\right) \times Z\left(\Omega_{B_{0}}^{u}\right)$, where the directional derivatives are 
defined as

$$
\begin{aligned}
D \mathcal{R}_{\boldsymbol{f}_{\boldsymbol{u}}}\left(\delta \boldsymbol{f}_{\boldsymbol{u}} ; \boldsymbol{u}^{n-1}, \boldsymbol{f}_{\boldsymbol{u}}, g_{\boldsymbol{u}}, h_{\boldsymbol{u}}, l_{\boldsymbol{u}}\right)\left[\Delta \boldsymbol{f}_{\boldsymbol{u}}\right] & =W_{K}^{u}\left(\boldsymbol{f}_{\boldsymbol{u}}, \delta \boldsymbol{f}_{\boldsymbol{u}}\right) G_{0}^{u}\left(g_{\boldsymbol{u}}, g_{\boldsymbol{u}}\right) H_{0}^{u}\left(h_{\boldsymbol{u}}, h_{\boldsymbol{u}}\right) L_{0}^{u}\left(l_{\boldsymbol{u}}, l_{\boldsymbol{u}}\right) \\
& +\mathrm{i} W_{C}^{u}\left(\boldsymbol{f}_{\boldsymbol{u}}, \delta \boldsymbol{f}_{\boldsymbol{u}}\right) G_{2}^{u}\left(g_{\boldsymbol{u}}, g_{\boldsymbol{u}}\right) H_{0}^{u}\left(h_{\boldsymbol{u}}, h_{\boldsymbol{u}}\right) L_{0}^{u}\left(l_{\boldsymbol{u}}, l_{\boldsymbol{u}}\right) \\
& -W_{M}^{u}\left(\boldsymbol{f}_{\boldsymbol{u}}, \delta \boldsymbol{f}_{\boldsymbol{u}}\right) G_{2}^{u}\left(g_{\boldsymbol{u}}, g_{\boldsymbol{u}}\right) H_{0}^{u}\left(h_{\boldsymbol{u}}, h_{\boldsymbol{u}}\right) L_{0}^{u}\left(l_{\boldsymbol{u}}, l_{\boldsymbol{u}}\right)
\end{aligned}
$$

$$
\begin{aligned}
D \mathcal{R}_{g_{\boldsymbol{u}}}\left(\delta g_{\boldsymbol{u}} ; \boldsymbol{u}^{n-1}, \boldsymbol{f}_{\boldsymbol{u}}, g_{\boldsymbol{u}}, h_{\boldsymbol{u}}, l_{\boldsymbol{u}}\right)\left[\Delta g_{\boldsymbol{u}}\right] & =W_{K}^{u}\left(\boldsymbol{f}_{\boldsymbol{u}}, \boldsymbol{f}_{\boldsymbol{u}}\right) G_{0}^{u}\left(g_{\boldsymbol{u}}, \delta g_{\boldsymbol{u}}\right) H_{0}^{u}\left(h_{\boldsymbol{u}}, h_{\boldsymbol{u}}\right) L_{0}^{u}\left(l_{\boldsymbol{u}}, l_{\boldsymbol{u}}\right) \\
& +\mathrm{i} W_{C}^{u}\left(\boldsymbol{f}_{\boldsymbol{u}}, \boldsymbol{f}_{\boldsymbol{u}}\right) G_{2}^{u}\left(g_{\boldsymbol{u}}, \delta g_{\boldsymbol{u}}\right) H_{0}^{u}\left(h_{\boldsymbol{u}}, h_{\boldsymbol{u}}\right) L_{0}^{u}\left(l_{\boldsymbol{u}}, l_{\boldsymbol{u}}\right) \\
& -W_{M}^{u}\left(\boldsymbol{f}_{\boldsymbol{u}}, \boldsymbol{f}_{\boldsymbol{u}}\right) G_{2}^{u}\left(g_{\boldsymbol{u}}, \delta g_{\boldsymbol{u}}\right) H_{0}^{u}\left(h_{\boldsymbol{u}}, h_{\boldsymbol{u}}\right) L_{0}^{u}\left(l_{\boldsymbol{u}}, l_{\boldsymbol{u}}\right)
\end{aligned}
$$

$$
\begin{aligned}
D \mathcal{R}_{h_{\boldsymbol{u}}}\left(\delta h_{\boldsymbol{u}} ; \boldsymbol{u}^{n-1}, \boldsymbol{f}_{\boldsymbol{u}}, g_{\boldsymbol{u}}, h_{\boldsymbol{u}}, l_{\boldsymbol{u}}\right)\left[\Delta h_{\boldsymbol{u}}\right] & =W_{K}^{u}\left(\boldsymbol{f}_{\boldsymbol{u}}, \boldsymbol{f}_{\boldsymbol{u}}\right) G_{0}^{u}\left(g_{\boldsymbol{u}}, g_{\boldsymbol{u}}\right) H_{0}^{u}\left(h_{\boldsymbol{u}}, \delta h_{\boldsymbol{u}}\right) L_{0}^{u}\left(l_{\boldsymbol{u}}, l_{\boldsymbol{u}}\right) \\
& +\mathrm{i} W_{C}^{u}\left(\boldsymbol{f}_{\boldsymbol{u}}, \boldsymbol{f}_{\boldsymbol{u}}\right) G_{2}^{u}\left(g_{\boldsymbol{u}}, g_{\boldsymbol{u}}\right) H_{0}^{u}\left(h_{\boldsymbol{u}}, \delta h_{\boldsymbol{u}}\right) L_{0}^{u}\left(l_{\boldsymbol{u}}, l_{\boldsymbol{u}}\right) \\
& -W_{M}^{u}\left(\boldsymbol{f}_{\boldsymbol{u}}, \boldsymbol{f}_{\boldsymbol{u}}\right) G_{2}^{u}\left(g_{\boldsymbol{u}}, g_{\boldsymbol{u}}\right) H_{0}^{u}\left(h_{\boldsymbol{u}}, \delta h_{\boldsymbol{u}}\right) L_{0}^{u}\left(l_{\boldsymbol{u}}, l_{\boldsymbol{u}}\right)
\end{aligned}
$$

$$
\begin{aligned}
D \mathcal{R}_{l_{\boldsymbol{u}}}\left(\delta l_{\boldsymbol{u}} ; \boldsymbol{u}^{n-1}, \boldsymbol{f}_{\boldsymbol{u}}, g_{\boldsymbol{u}}, h_{\boldsymbol{u}}, l_{\boldsymbol{u}}\right)\left[\Delta l_{\boldsymbol{u}}\right] & =W_{K}^{u}\left(\boldsymbol{f}_{\boldsymbol{u}}, \boldsymbol{f}_{\boldsymbol{u}}\right) G_{0}^{u}\left(g_{\boldsymbol{u}}, g_{\boldsymbol{u}}\right) H_{0}^{u}\left(h_{\boldsymbol{u}}, h_{\boldsymbol{u}}\right) L_{0}^{u}\left(l_{\boldsymbol{u}}, \delta l_{\boldsymbol{u}}\right) \\
& +\mathrm{i} W_{C}^{u}\left(\boldsymbol{f}_{\boldsymbol{u}}, \boldsymbol{f}_{\boldsymbol{u}}\right) G_{2}^{u}\left(g_{\boldsymbol{u}}, g_{\boldsymbol{u}}\right) H_{0}^{u}\left(h_{\boldsymbol{u}}, h_{\boldsymbol{u}}\right) L_{0}^{u}\left(l_{\boldsymbol{u}}, \delta l_{\boldsymbol{u}}\right) \\
& -W_{M}^{u}\left(\boldsymbol{f}_{\boldsymbol{u}}, \boldsymbol{f}_{\boldsymbol{u}}\right) G_{2}^{u}\left(g_{\boldsymbol{u}}, g_{\boldsymbol{u}}\right) H_{0}^{u}\left(h_{\boldsymbol{u}}, h_{\boldsymbol{u}}\right) L_{0}^{u}\left(l_{\boldsymbol{u}}, \delta l_{\boldsymbol{u}}\right)
\end{aligned}
$$

The standard Galerkin FEM discretisation process [11] is used in order to obtain the discrete solution of the mechanical problem. Thus, equations (A.12) are expressed in their discretised version as

$$
\begin{aligned}
&\left(g_{0}^{u} h_{0}^{u} l_{0}^{u} \mathbf{K}^{u}+\mathrm{i} g_{2}^{u} h_{0}^{u} l_{0}^{u} \mathbf{C}^{u}-g_{2}^{u} h_{0}^{u} l_{0}^{u} \mathbf{M}^{u}\right) \Delta \mathbf{f}_{\boldsymbol{u}}=-\mathbf{R}_{\boldsymbol{f}_{\boldsymbol{u}}}\left(\boldsymbol{f}_{\boldsymbol{u}}, g_{\boldsymbol{u}}, h_{\boldsymbol{u}}, l_{\boldsymbol{u}}\right), \\
&\left(k^{u} h_{0}^{u} l_{0}^{u} \mathbf{G}_{0}^{u}+\mathrm{i} c^{u} h_{0}^{u} l_{0}^{u} \mathbf{G}_{2}^{u}-m^{u} h_{0}^{u} l_{0}^{u} \mathbf{G}_{2}^{u}\right) \Delta \mathbf{g}_{\boldsymbol{u}}=-\mathbf{R}_{g_{\boldsymbol{u}}}\left(\boldsymbol{f}_{\boldsymbol{u}}, g_{\boldsymbol{u}}, h_{\boldsymbol{u}}, l_{\boldsymbol{u}}\right), \\
&\left(k^{u} g_{0}^{u} l_{0}^{u} \mathbf{H}_{0}^{u}+\mathrm{i} c^{u} g_{2}^{u} l_{0}^{u} \mathbf{H}_{0}^{u}-m^{u} g_{2}^{u} l_{0}^{u} \mathbf{H}_{0}^{u}\right) \Delta \mathbf{h}_{\boldsymbol{u}}=-\mathbf{R}_{h_{\boldsymbol{u}}}\left(\boldsymbol{f}_{\boldsymbol{u}}, g_{\boldsymbol{u}}, h_{\boldsymbol{u}}, l_{\boldsymbol{u}}\right) \\
&\left(k^{u} g_{0}^{u} h_{0}^{u} \mathbf{L}_{0}^{u}+\mathrm{i} c^{u} g_{2}^{u} h_{0}^{u} \mathbf{L}_{0}^{u}-m^{u} g_{2}^{u} h_{0}^{u} \mathbf{L}_{0}^{u}\right) \Delta \mathbf{l}_{\boldsymbol{u}}=-\mathbf{R}_{l_{\boldsymbol{u}}}\left(\boldsymbol{f}_{\boldsymbol{u}}, g_{\boldsymbol{u}}, h_{\boldsymbol{u}}, l_{\boldsymbol{u}}\right)
\end{aligned}
$$

with

$$
\begin{aligned}
\mathbf{R}_{\boldsymbol{f}_{\boldsymbol{u}}}\left(\boldsymbol{f}_{\boldsymbol{u}}, g_{\boldsymbol{u}}, h_{\boldsymbol{u}}, l_{\boldsymbol{u}}\right) & =\left(g_{0}^{u} h_{0}^{u} l_{0}^{u} \mathbf{K}^{u}+\mathrm{i} g_{2}^{u} h_{0}^{u} l_{0}^{u} \mathbf{C}^{u}-g_{2}^{u} h_{0}^{u} l_{0}^{u} \mathbf{M}^{u}\right) \mathbf{f}_{\boldsymbol{u}} \\
& +\sum_{m=1}^{N_{A}}\left[g_{0, s}^{u,[m]} h_{0, s}^{u,[m]} l_{1, s}^{u,[m]} \mathbf{K}^{u A}\right] \mathbf{F}_{\mathcal{A}_{\phi}}^{[m]} \\
& +\sum_{m=1}^{n-1}\left[g_{0}^{u,[m]} h_{0}^{u,[m]} l_{0}^{u,[m]} \mathbf{K}^{u}+\mathrm{i} g_{2}^{u,[m]} h_{0}^{u,[m]} l_{0}^{u,[m]} \mathbf{C}^{u}\right. \\
& \left.-g_{2}^{u,[m]} h_{0}^{u,[m]} l_{0}^{u,[m]} \mathbf{M}^{u}\right] \mathbf{F}_{\boldsymbol{u}}^{[m]}, \\
\mathbf{R}_{g_{\boldsymbol{u}}}\left(\boldsymbol{f}_{\boldsymbol{u}}, g_{\boldsymbol{u}}, h_{\boldsymbol{u}}, l_{\boldsymbol{u}}\right)= & \left(k^{u} h_{0}^{u} l_{0}^{u} \mathbf{G}_{0}^{u}+\mathrm{i} c^{u} h_{0}^{u} l_{0}^{u} \mathbf{G}_{2}^{u}-m^{u} h_{0}^{u} l_{0}^{u} \mathbf{G}_{2}^{u}\right) \mathbf{g}_{\boldsymbol{u}} \\
+ & \sum_{m=1}^{N_{A}}\left[k_{s}^{u,[m]} h_{0, s}^{u,[m]} l_{1, s}^{u,[m]} \mathbf{G}_{0}^{u}\right] \mathbf{G}_{\mathcal{A}_{\phi}}^{[m]} \\
+ & \sum_{m=1}^{n-1}\left[k^{u,[m]} h_{0}^{u,[m]} l_{0}^{u,[m]} \mathbf{G}_{0}^{u}+\mathrm{i} c^{u,[m]} h_{0}^{u,[m]} l_{0}^{u,[m]} \mathbf{G}_{2}^{u}\right. \\
& \left.-m^{u,[m]} h_{0}^{u,[m]} l_{0}^{u,[m]} \mathbf{G}_{2}^{u}\right] \mathbf{G}_{\boldsymbol{u}}^{[m]},
\end{aligned}
$$




$$
\begin{aligned}
\mathbf{R}_{h_{\boldsymbol{u}}}\left(\boldsymbol{f}_{\boldsymbol{u}}, g_{\boldsymbol{u}}, h_{\boldsymbol{u}}, l_{\boldsymbol{u}}\right) & =\left(k^{u} g_{0}^{u} l_{0}^{u} \mathbf{H}_{0}^{u}+\mathrm{i} c^{u} g_{2}^{u} l_{0}^{u} \mathbf{H}_{0}^{u}-m^{u} g_{2}^{u} l_{0}^{u} \mathbf{H}_{0}^{u}\right) \mathbf{h}_{\boldsymbol{u}} \\
& +\sum_{m=1}^{N_{A}}\left[k_{s}^{u,[m]} g_{0, s}^{u,[m]} l_{1, s}^{u,[m]} \mathbf{H}_{0}^{u}\right] \mathbf{H}_{\mathcal{A}_{\phi}}^{[m]} \\
& +\sum_{m=1}^{n-1}\left[k^{u,[m]} g_{0}^{u,[m]} l_{0}^{u,[m]} \mathbf{H}_{0}^{u}+\mathrm{i} c^{u,[m]} g_{2}^{u,[m]} l_{0}^{u,[m]} \mathbf{H}_{0}^{u}\right. \\
& \left.-m^{u,[m]} g_{2}^{u,[m]} l_{0}^{u,[m]} \mathbf{H}_{0}^{u}\right] \mathbf{H}_{\boldsymbol{u}}^{[m]}, \\
\mathbf{R}_{l_{\boldsymbol{u}}}\left(\boldsymbol{f}_{\boldsymbol{u}}, g_{\boldsymbol{u}}, h_{\boldsymbol{u}}, l_{\boldsymbol{u}}\right)= & \left(k^{u} g_{0}^{u} h_{0}^{u} \mathbf{L}_{0}^{u}+\mathrm{i} c^{u} g_{2}^{u} h_{0}^{u} \mathbf{L}_{0}^{u}-m^{u} g_{2}^{u} h_{0}^{u} \mathbf{L}_{0}^{u}\right) \mathbf{l}_{\boldsymbol{u}} \\
& +\sum_{m=1}^{N_{A}}\left[k_{s}^{u,[m]} g_{0, s}^{u,[m]} h_{0, s}^{u,[m]} \mathbf{L}_{1}^{u}\right] \mathbf{L}_{\mathcal{A}_{\phi}}^{[m]} \\
& +\sum_{m=1}^{n-1}\left[k^{u,[m]} g_{0}^{u,[m]} h_{0}^{u,[m]} \mathbf{L}_{0}^{u}+\mathrm{i} c^{u,[m]} g_{2}^{u,[m]} h_{0}^{u,[m]} \mathbf{L}_{0}^{u}\right. \\
& \left.-m^{u,[m]} g_{2}^{u,[m]} h_{0}^{u,[m]} \mathbf{L}_{0}^{u}\right] \mathbf{L}_{\boldsymbol{u}}^{[m]},
\end{aligned}
$$

and where the scalar quantities are defined as

$$
\begin{aligned}
& h_{0}^{u}:=H_{0}^{u}\left(h_{\boldsymbol{u}}, h_{\boldsymbol{u}}\right),
\end{aligned}
$$

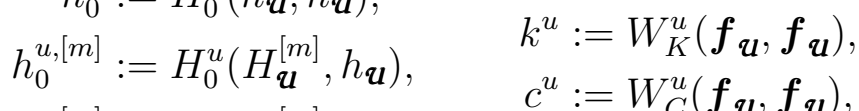

$$
\begin{aligned}
& g_{0}^{u}:=G_{0}^{u}\left(g_{\boldsymbol{u}}, g_{\boldsymbol{u}}\right), \\
& g_{2}^{u}:=G_{2}^{u}\left(g_{\boldsymbol{u}}, g_{\boldsymbol{u}}\right) \text {, } \\
& h_{0, s}^{u,[m]}:=H_{0}^{u}\left(H_{\mathfrak{A}_{\phi}}^{[m]}, h_{\boldsymbol{u}}\right), \\
& m^{u}:=W_{M}^{u}\left(\boldsymbol{f}_{\boldsymbol{u}}, \boldsymbol{f}_{\boldsymbol{u}}\right), \\
& g_{0}^{u,[m]}:=G_{0}^{u}\left(G_{\boldsymbol{u}}^{[m]}, g_{\boldsymbol{u}}\right), \\
& g_{2}^{u,[m]}:=G_{2}^{u}\left(G_{\boldsymbol{u}}^{[m]}, g_{\boldsymbol{u}}\right), \\
& g_{0, s}^{u,[m]}:=G_{0}^{u}\left(G_{\mathfrak{A}_{\phi}}^{[m]}, g_{\boldsymbol{u}}\right), \\
& l_{0}^{u}:=L_{0}^{u}\left(l_{\boldsymbol{u}}, l_{\boldsymbol{u}}\right), \\
& l_{1}^{u}:=L_{1}^{u}\left(l_{\boldsymbol{u}}, l_{\boldsymbol{u}}\right), \\
& l_{0}^{u,[m]}:=L_{0}^{u}\left(L_{\boldsymbol{u}}^{[m]}, l_{\boldsymbol{u}}\right), \\
& l_{1}^{u,[m]}:=L_{1}^{u}\left(L_{\boldsymbol{u}}^{[m]}, l_{\boldsymbol{u}}\right), \\
& l_{1, s}^{u,[m]}:=L_{1}^{u}\left(L_{\mathfrak{A}_{\phi}}^{[m]}, l_{\boldsymbol{u}}\right),
\end{aligned}
$$

Note that the integration of the quantities $G_{0}^{u}\left(G_{\mathcal{A}_{\phi}}^{[m]}, g_{\boldsymbol{u}}\right), H_{0}^{u}\left(H_{\mathfrak{A}_{\phi}}^{[m]}, h_{\boldsymbol{u}}\right)$ and $L_{1}^{u}\left(L_{\mathfrak{A}_{\phi}}^{[m]}, l_{\boldsymbol{u}}\right)$ involves the use of one-dimensional mortar integrals which are detailed in Appendix B.

\section{Appendix B. One-dimensional mortar integral treatment}

Some of the terms featuring in (A.15) require the computation of an integral that can potentially involve different non-matching one-dimensional meshes. In the context of friction and contact-impact problems [52-54], this type of integrals are known as mortar integrals and this Appendix provides a detailed explanation of the implementation for the one-dimensional case used in this paper. As an example, let us consider the evaluation of $g_{0}^{u A,[m]}$. As it appears in (A.16), the definition of this coefficient is

$$
g_{0, s}^{u,[m]}:=G_{0}^{u}\left(G_{\mathcal{A}_{\phi}}^{[m]}, g_{\boldsymbol{u}}\right)=\overline{\mathbf{g}}_{\boldsymbol{u}}^{T} \mathbf{G}_{0}^{u} \mathbf{G}_{\mathcal{A}_{\phi}}^{[m]},
$$

where the product $\overline{\mathbf{g}}_{\boldsymbol{u}}^{T} \mathbf{G}_{0}^{u} \mathbf{G}_{\mathfrak{A}_{\phi}}^{[m]}$ may require the assembly of a rectangular mass type matrix, allowing the use of different meshes with different number of degrees of freedom for both electromagnetics and mechanics fields. Note that for the case where the electromagnetic parametric mesh coincides with the mechanical one, then $\mathbf{G}_{0}^{u}=\mathbf{G}_{0}^{A}$ which coincides with the standard onedimensional consistent mass matrix for both physics. In general,

$$
G_{0}^{u}\left(G_{\mathfrak{A}_{\phi}}^{[m]}, g_{\boldsymbol{u}}\right)=\int_{\Omega_{\omega}^{u}} G_{\mathcal{A}_{\phi}}^{[m]}(\omega) \bar{g}_{\boldsymbol{u}}(\omega) \mathrm{d} \omega
$$


where the two integrands are discretised as

$$
g_{\boldsymbol{u}}=\sum_{a=1}^{Q_{\omega}^{u}} N_{\boldsymbol{u}}^{a} g_{\mathfrak{u}}^{a}, \quad G_{\mathfrak{A}_{\phi}}^{[m]}=\sum_{b=1}^{Q_{\omega}^{A}} N_{\mathfrak{A}_{\phi}}^{b} G_{\mathfrak{A}_{\phi}}^{b,[m]}
$$

with $Q_{\omega}^{u}$ and $Q_{\omega}^{A}$ being the total number of degrees of freedom of the frequency domain for mechanics and electromagnetics, respectively. The integral can then be written as

$$
G_{0}^{u}\left(G_{\mathcal{A}_{\phi}}^{[m]}, g_{\mathfrak{u}}\right)=\sum_{a=1}^{Q_{\omega}^{u}} \sum_{b=1}^{Q_{\omega}^{A}} \bar{g}_{\mathfrak{u}}^{a} \underbrace{\int_{\Omega_{\omega}^{u,(e)}} N_{\mathfrak{u}^{a}}^{a} N_{\mathcal{A}_{\phi}}^{b} \mathrm{~d} \omega}_{=m_{a b}} G_{\mathcal{A}_{\phi}}^{b,[m]},
$$

and, thus, a particular $a b$ entry of $\mathbf{G}_{0}^{u,(e)}$ is computed using a Gaussian quadrature as

$$
m_{a b}=\sum_{i=1}^{Q_{g p}} N_{\mathfrak{u}}^{a}\left(\zeta_{i}\right) N_{\mathcal{A}_{\phi}}^{b}\left(\zeta_{i}\right) J_{i} w_{i}
$$

where $\zeta_{i}, J_{i}$ and $w_{i}$ are the position of the Gauss points, the Jacobian and weights of the Gauss points, respectively, which are all related to the mechanical frequency domain since the integral is over $\Omega_{\omega}^{u}$. The computation of this term require careful consideration in the case of nonmatching meshes in the parametric domain (for the electromagnetics and mechanical physics), see Figure B.17. For the simple one-dimensional case, the necessary steps are summarised in Algorithm 1.
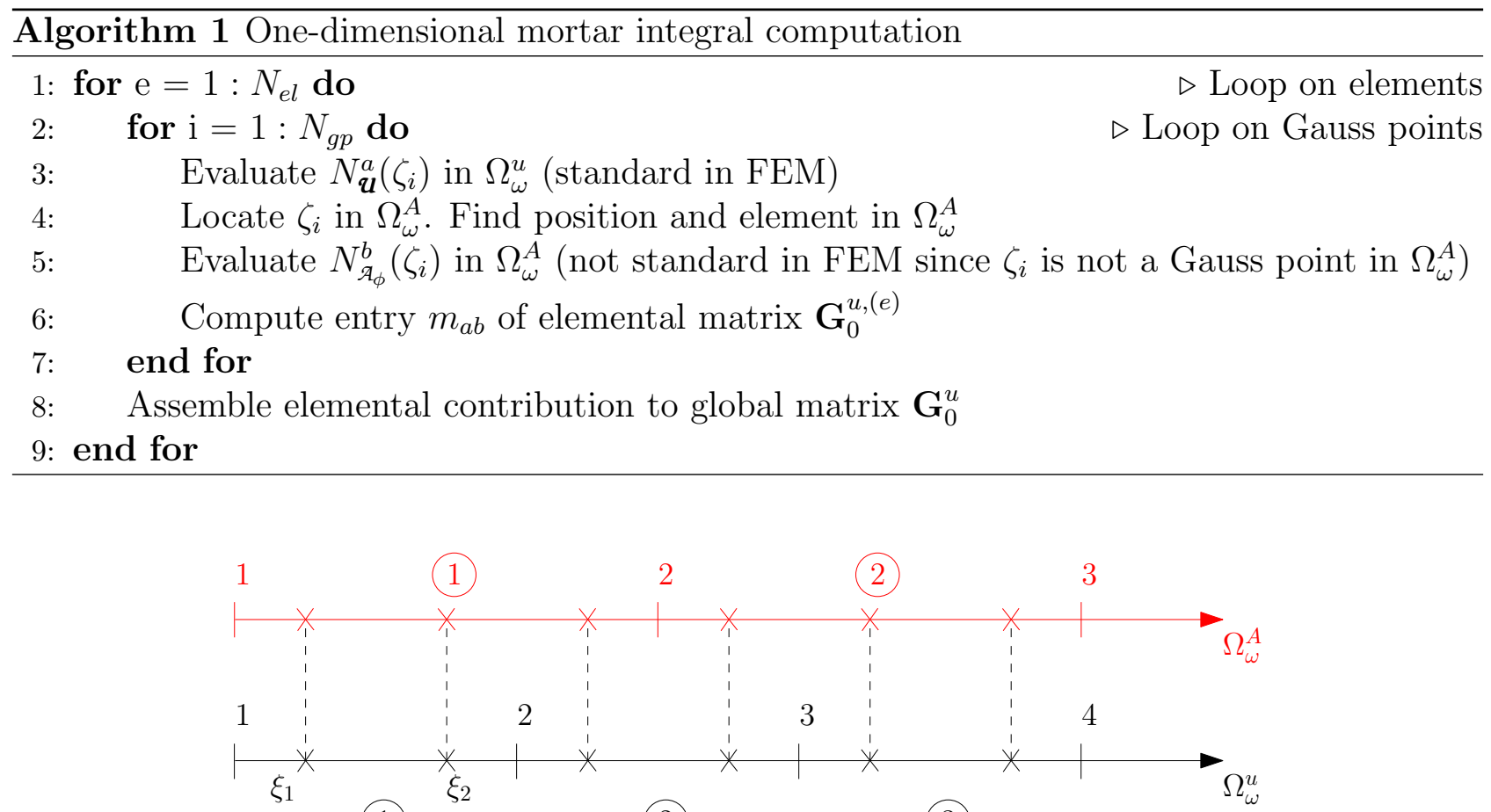

(1)

(2)

(3)

Figure B.17: Representation of two non-matching one-dimensional meshes.

Using Figure B.17 as an example of two different one-dimensional meshes, the three elemental matrices in $\Omega_{\omega}^{u}$ have the following structure

$$
\mathbf{G}_{0}^{u,(1)}=\left[\begin{array}{ll}
m_{11} & m_{12} \\
m_{21} & m_{22}
\end{array}\right], \quad \mathbf{G}_{0}^{u,(2)}=\left[\begin{array}{ccc}
m_{21} & m_{22} & 0 \\
0 & m_{32} & m_{33}
\end{array}\right], \quad \mathbf{G}_{0}^{u,(3)}=\left[\begin{array}{ll}
m_{32} & m_{33} \\
m_{42} & m_{43}
\end{array}\right],
$$


which are assembled in the global matrix as

$$
\mathbf{G}_{0}^{u}=\left[\begin{array}{ccc}
m_{11} & m_{12} & 0 \\
m_{21} & m_{22} & 0 \\
0 & m_{32} & m_{33} \\
0 & m_{42} & m_{43}
\end{array}\right]
$$

\section{References}

[1] L. Clarke, R. Velthuizen, M. Camacho, J. Heine, M. Vaidyanathan, L. Hall, R. Thatcher, M. Silbiger, MRI segmentation: methods and applications, Magnetic Resonance Imaging 13 (1995) 343-368.

[2] S. J. Matzat, J. van Tiel, G. E. Gold, E. H. Oei, Quantitative MRI techniques of cartilage composition, Quantitative Imaging in Medicine and Surgery 3 (2013) 162.

[3] A. Savnik, H. Malmskov, H. S. Thomsen, L. B. Graff, H. Nielsen, B. Danneskiold-Samsøe, J. Boesen, H. Bliddal, MRI of the wrist and finger joints in inflammatory joint diseases at 1-year interval: MRI features to predict bone erosions, European Radiology 12 (2002) 1203-1210.

[4] P. Kozlowski, S. D. Chang, E. C. Jones, K. W. Berean, H. Chen, S. L. Goldenberg, Combined diffusion-weighted and dynamic contrast-enhanced MRI for prostate cancer diagnosis: Correlation with biopsy and histopathology, Journal of Magnetic Resonance Imaging: An Official Journal of the International Society for Magnetic Resonance in Medicine 24 (2006) 108-113.

[5] J. A. Pope, Medical Physics: Imaging, Heinemann, Portsmouth, United States, 1999.

[6] R. Aarnink, J. Overweg, Magnetic resonance imaging, a success story for superconductivity, Europhysics News 43 (2012) 26-29.

[7] A. Buffa, H. Ammari, J.-C. Nédélec, A justification of eddy currents model for the Maxwell equations, SIAM Journal on Applied Mathematics 60 (2000) 1805-1823.

[8] A. A. Rodríguez, A. Valli, Eddy Current Approximation of Maxwell Equations: Theory, Algorithms and Applications, volume 4, Springer Science \& Business Media, Berlin, Germany, 2010.

[9] S. Kurz, J. Fetzer, G. Lehner, W. M. Rucker, A novel formulation for 3D eddy current problems with moving bodies using a Lagrangian description and BEM-FEM coupling, IEEE Transactions on Magnetics 34 (1998) 3068-3073.

[10] P. D. Ledger, S. Zaglmayr, hp-Finite element simulation of three-dimensional eddy current problems on multiply connected domains, Computer Methods in Applied Mechanics and Engineering 199 (2010) 3386-3401.

[11] T. J. R. Hughes, The Finite Element Method: Linear Static and Dynamic Finite Element Analysis, Courier Corporation, Massachusetts, United States, 2012.

[12] A. J. Gil, P. D. Ledger, M. Seoane, G. Barroso, M. Mallett, In preparation (2019).

[13] P. D. Ledger, A. J. Gil, R. Poya, M. Kruip, I. Wilkinson, S. Bagwell, Solution of an industrially relevant coupled magneto-mechanical problem set on an axisymmetric domain, Applied Mathematical Modelling 40 (2016) 1959-1971. 
[14] S. Bagwell, P. D. Ledger, A. J. Gil, M. Mallett, M. Kruip, A linearised hp-finite element framework for acousto-magneto-mechanical coupling in axisymmetric MRI scanners, International Journal for Numerical Methods in Engineering 112 (2017) 1323-1352.

[15] S. Bagwell, P. D. Ledger, A. J. Gil, M. Mallett, Transient solutions to nonlinear acoustomagneto-mechanical coupling for axisymmetric MRI scanner design, International Journal for Numerical Methods in Engineering 115 (2018) 209-237.

[16] M. Seoane, P. D. Ledger, A. J. Gil, M. Mallett, An accurate and efficient three-dimensional high-order finite element methodology for the simulation of magneto-mechanical coupling in MRI scanners, International Journal for Numerical Methods in Engineering (Accepted 2019). 10.1002/nme.6088.

[17] Siemens Healthineers. Magnetic resonance imaging, https://www .healthcare. siemens . co.uk/news/smt-7t.html, 2019. Accessed: 27/03/2019.

[18] D. Amsallem, C. Farhat, Interpolation method for adapting reduced-order models and application to aeroelasticity, AIAA Journal 46 (2008) 1803-1813.

[19] F. Chinesta, R. Keunings, A. Leygue, The Proper Generalized Decomposition for Advanced Numerical Simulations: A Primer, Springer Science \& Business Media, Berlin, Germany, 2013.

[20] G. Rozza, D. B. P. Huynh, A. T. Patera, Reduced basis approximation and a posteriori error estimation for affinely parametrized elliptic coercive partial differential equations, Archives of Computational Methods in Engineering (2008). 15: 229. https://doi.org/10.1007/s11831-008-9019-9.

[21] Y. Liang, H. Lee, S. Lim, W. Lin, K. Lee, C. Wu, Proper orthogonal decomposition and its applications. Part i: Theory, Journal of Sound and Vibration 252 (2002) 527-544.

[22] T. R. Smith, J. Moehlis, P. Holmes, Low-dimensional modelling of turbulence using the proper orthogonal decomposition: a tutorial, Nonlinear Dynamics 41 (2005) 275-307.

[23] P. Díez, S. Zlotnik, A. García-González, A. Huerta, Algebraic PGD for tensor separation and compression: An algorithmic approach, Comptes Rendus Mécanique 346 (2018) 501514.

[24] P. Díez, S. Zlotnik, A. Huerta, Generalized parametric solutions in Stokes flow, Computer Methods in Applied Mechanics and Engineering 326 (2017) 223-240.

[25] D. Modesto, S. Zlotnik, A. Huerta, Proper Generalized Decomposition for parameterized Helmholtz problems in heterogeneous and unbounded domains: Application to harbor agitation, Computer Methods in Applied Mechanics and Engineering 295 (2015) 127-149.

[26] M. Signorini, S. Zlotnik, P. Diez, Proper generalized decomposition solution of the parameterized helmholtz problem: application to inverse geophysical problems, International Journal for Numerical Methods in Engineering 109 (2017) 1085-1102.

[27] J. M. de Almeida, A basis for bounding the errors of proper generalised decomposition solutions in solid mechanics, International Journal for Numerical Methods in Engineering 94 (2013) 961-984.

[28] R. García-Blanco, D. Borzacchiello, F. Chinesta, P. Diez, Monitoring a PGD solver for parametric power flow problems with goal-oriented error assessment, International Journal for Numerical Methods in Engineering 111 (2017) 529-552. 
[29] R. Ibáñez, E. Abisset-Chavanne, F. Chinesta, A. Huerta, Simulating squeeze flows in multiaxial laminates: towards fully 3d mixed formulations, International Journal Of Material Forming 10 (2017) 653-669.

[30] V. Tsiolakis, M. Giacomini, R. Sevilla, C. Othmer, A. Huerta, Nonintrusive proper generalised decomposition for parametrised incompressible flow problems in OpenFOAM, arXiv preprint arXiv:1906.05403 (2019).

[31] C. Ghnatios, F. Masson, A. Huerta, A. Leygue, E. Cueto, F. Chinesta, Proper generalized decomposition based dynamic data-driven control of thermal processes, Computer Methods in Applied Mechanics and Engineering 213 (2012) 29-41.

[32] J. V. Aguado, A. Huerta, F. Chinesta, E. Cueto, Real-time monitoring of thermal processes by reduced-order modeling, International Journal for Numerical Methods in Engineering 102 (2015) 991-1017.

[33] B. Bognet, A. Leygue, F. Chinesta, On the fully 3D simulations of thermoelastic models defined in plate and shell geometries, European Journal of Computational Mechanics/Revue Européenne de Mécanique Numérique 21 (2012) 40-51.

[34] S. Zlotnik, P. Díez, D. Modesto, A. Huerta, Proper Generalized Decomposition of a geometrically parametrized heat problem with geophysical applications, International Journal for Numerical Methods in Engineering 103 (2015) 737-758.

[35] G. Barroso, A. J. Gil, P. D. Ledger, M. Mallett, A. Huerta, A regularised-adaptive Proper Generalised Decomposition implementation for coupled magneto-mechanical problems with application to MRI scanners 358 (112640) (2020).

[36] M. Seoane, P. D. Ledger, A. J. Gil, S. Zlotnik, M. Mallett, A combined reduced order-full order methodology for the solution of 3D magneto-mechanical problems with application to MRI scanners (2020).

[37] S. Bagwell, A Numerical Multi-Physics Approach to Understanding MRI Scanners and their Complex Behaviour, Ph.D. thesis, Swansea University, UK, 2018.

[38] S. M. Nazeer, F. Bordeu, A. Leygue, F. Chinesta, Arlequin based PGD domain decomposition, Computational Mechanics 54 (2014) 1175-1190.

[39] F. Chinesta, P. Ladevèze, Separated Representations and PGD-Based Model Reduction, Fundamentals and Applications, International Centre for Mechanical Siences, Courses and Lectures 554 (2014).

[40] J. Bonet, A. J. Gil, R. D. Wood, Nonlinear Solid Mechanics for Finite Element Analysis: Statics, Cambridge University Press, United Kingdom, 2016.

[41] A. Chatterjee, An introduction to the proper orthogonal decomposition, Current science (2000) 808-817.

[42] V. Buljak, Inverse Analyses with Model Reduction: Proper Orthogonal Decomposition in Structural Mechanics, Springer Science \& Business Media, Berlin, Germany, 2011.

[43] A. Bjorck, Numerical Methods For Least Squares Problems, SIAM, Philadelphia, United States, 1996.

[44] P. C. Hansen, Rank-Deficient And Discrete Ill-Posed Problems: Numerical Aspects Of Linear Inversion, SIAM, Philadelphia, United States, 2005. 
[45] L. De Lathauwer, B. De Moor, J. Vandewalle, A multilinear singular value decomposition, SIAM journal on Matrix Analysis and Applications 21 (2000) 1253-1278.

[46] T. Bui-Thanh, M. Damodaran, K. Willcox, Proper orthogonal decomposition extensions for parametric applications in compressible aerodynamics, in: 21st AIAA Applied Aerodynamics Conference, Florida, p. 4213 (2003).

[47] R. Rama, Proper Orthogonal Decomposition With Interpolation-Based Real-Time Modelling Of The Heart, Ph.D. thesis, University of Cape Town, 2017.

[48] R. Rama, S. Skatulla, C. Sansour, Real-time modelling of the heart using the proper orthogonal decomposition with interpolation, in: VI International Conference on Computational Bioengineering (ICCB), pp. 1-12 (2015).

[49] N. Demo, N. Tezzele, G. Gustin, G. Lavini, G. Rozza, Shape optimisation by means of proper orthogonal decomposition and dynamic mode decomposition, arXiv preprint arXiv:1803.07368 (2018).

[50] S. Georgaka, G. Stabile, G. Rozza, M. Bluck, Parametric POD-Galerkin model order reduction for unsteady-state heat transfer problems, arXiv preprint arXiv:1808.05175 (2018).

[51] G. Stabile, S. Hijazi, A. Mola, S. Lorenzi, G. Rozza, POD-Galerkin reduced order methods for CFD using Finite Volume Discretisation: vortex shedding around a circular cylinder, Communications in Applied and Industrial Mathematics 8 (2017) 210-236.

[52] C. Hesch, A. J. Gil, A. A. Carreño, J. Bonet, P. Betsch, A mortar approach for fluid-structure interaction problems: Immersed strategies for deformable and rigid bodies, Computer Methods in Applied Mechanics and Engineering 278 (2014) 853 - 882.

[53] T. McDevitt, T. Laursen, A mortar-finite element formulation for frictional contact problems, International Journal for Numerical Methods in Engineering 48 (2000) 1525-1547.

[54] A. Popp, M. W. Gee, W. A. Wall, A finite deformation mortar contact formulation using a primal-dual active set strategy, International Journal for Numerical Methods in Engineering 79 (2009) 1354-1391. 